\title{
LES SUBSTANCES ANTIBACTÉRIENNES \\ DANS LA COLONIE D'ABEILLES \\ (APIS MELLIFICA L.) (fin)
}

par

P. LAVIE

Station expérimentale d'Apiculture, Monfavet

CHAPITRE, III

\section{L'ANTIBIOTIQUE DES POLLENS RÉCOLTÉS PAR APIS MELLIFICA I.}

\author{
A. - Généralités. \\ Io Récolte et utilisation.
}

Pour récolter le pollen, l'abeille doit confectionner des pelotes qu'elle transporte dans les corbeilles des pattes et cette opération nécessite l'agglutination de grains de pollen grâce à l'addition de sécrétions salivaires et d'une certaine quantité de nectar ou de miel. Nous verrons par la suite l'importance de cette addition de substances vis-à-vis du sujet qui nous préoccupe. ToDD et BRETHERICK (I942) ont montré que le pollen récolté par les abeilles contenait plus de sucres que le pollen des mêmes plantes récolté à la main. Dans les pelotes de pollen, le taux en sucres réducteurs peut atteindre de 20 à $40 \mathrm{p}$. Ioo du poids sec, tandis que le pollen récolté à la main ne dépasse pas 7,5 p. Ioo. Déjà Parker (I926) avait signalé que les abeilles récoltant des pollens faisaient avant leur départ de la ruche une provision de miel qui correspond à la moitié du volume du proventricule.

Le pollen est l'aliment plastique de la ruche, il a une grande importance non seulement sur le développement du couvain, mais également sur la durée de vie chez l'adulte. On peut dire que la quantité et la qualité 
de sa récolte, seule nourriture azotée dans la ruche, règle en grande partie le développement de la colonie. D'après Louveaux (1958), une récolte de 25 kilogrammes par an et par ruche constitue la ration moyenne, mais des écarts très importants existent d'une colonie à une autre. Cet auteur précise également son rôle essentiel dans l'état physiologique du groupe d'abeilles pris dans son ensemble. L'abeille choisit les pollens dans son rayon de butinage et ne visite qu'un nombre de plantes réduit (Synge, I947). De nombreux auteurs (cités par Louveaux, 1958) ont étudié la composition chimique des pollens. Celle-ci est très variable et il est certain que de nombreux pollens ont des valeurs biologiques très inégales. Par exemple on sait que les pollens de conifères étudiés par Madrizio (I954) sont de faible valeur biologique, peu recherchés par les abeilles et butinés seulement en cas de disette. On admet qu'un mélange de pollens variés apporte aux abeilles tous les éléments nécessaires à leur développement (acides aminés et protéines, lipides, vitamines, oligoéléments). Je montrerai d'ailleurs qu'au point de vue de l'antibiotique, les pollens récoltés par les abeilles ne contiemnent pas tous une substance antibiotique et, lorsqu'elle existe, son activité est très variable. Mais dans un mélange de pollens variés récoltés par les abeilles, il est toujours possible d'extraire un antibiotique d'activité à peu près constante. Nous allons maintenant essayer de trouver dans la bibliographie les travaux préliminaires qui ont mis à jour l'activité antibactérienne des pollens récoltés par les abeilles.

\section{$2^{\circ}$ Travaux antérieurs concernant l'activité antibactérienne du pollen.}

WhiTE (I906), dans ses recherches sur la flore bactérienne de la ruche, fut le premier à étudier la flore bactérienne trouvée dans le pollen récolté par les abeilles. Le protocole des expériences ne précise pas s'il s'agit de pelotes de pollen recueillies à l'entrée des butineuses ou de pollen stocké dans les cellules. Cependant, d'après la date et l'ensemble du travail, je suppose qu'il s'agit de pollen emmagasiné. L'auteur précise que le nombre d'espèces bactériennes qu'on y rencontre est très petit et même plus faible que pour les espèces trouvées sur les rayons de cire. Il retrouve souvent un bacille appelé Bacillus $B$ qui est présent également dans 1'intestin de 1'abeille adulte. Il a noté encore quelques espèces bactériennes, mais dont la présence est seulement occasionnelle. Nous essaierons de voir par la suite les rapports possibles de cette bactérie avec la maturation du pollen stocké dans les rayons.

D'autre part, si nous considérons les nombreux travaux se rapportant à la nutrition de Galleria mellonella, on peut se demander si 1'immunité de la chenille vis-à-vis du bacille de Koch n'est pas due en partie à 
l'ingestion de pollen. Les chenilles de la fausse teigne se nourrissent de cire, mais également d'une grande quantité de matière azotée (pollen, exuvies de larves d'abeilles, etc.). Dans l'intestin la présence de lipase et d'une protéase confirme ce mode d'alimentation (Roy, 1937). Ces insectes nourris avec de la cire pure sont incapables de boucler leur cycle normal, mais d'autre part un régime privé de cire est insuffisant pour le développement de la larve. Toutes ces expériences montrent la necessité des deux substances pour le développement de Galleria mellonella. Nous avions émis 1'hypothèse dans le chapitre précédent que la dégradation de la cire dans l'intestin de la larve pouvait libérer un antibiotique actif sur le bacille de Koch. On peut se demander si l'action d'un antibiotique présent dans le pollen des ruches n'entre pas également en jeu. Nous avons vu également que le pollen est présent en quantité non négligeable dans la cire des rayons.

Smith, Beck et Anderson (I949) ont étudié dans le pollen une substance accélératrice de la sporulation chez Bacillus larvae. Ce facteur est hydrosoluble et thermostable. L'extrait aqueux était obtenu de la manière suivante : broyage au mortier avec de l'eau distillée pendant 20 minutes, agitation, filtration sur Büchner. La liqueur était additionnée au milieu de culture et le $\mathrm{pH}$ ajusté à 6,8-7 avec de la soude puis le tout stérilisé à $\mathrm{I}_{20^{\circ}}$ pendant $\mathrm{I}_{5}$ minutes. Les résultats obtenus sont les suivants :

Les spores augmentent dans les milieux de culture si la quantité d'extrait de pollen augmente.

2 p. roo de pollen est le pourcentage optima pour la sporulation.

Si l'on ajoute plus de $3 \mathrm{p}$. Ioo d'extrait, l'inhibition du bacille est totale. Ces résultats sont à rapprocher des observations faites par certains apiculteurs dans leurs ruchers. Il est en effet bien connu et vérifié que les maladies du couvain peuvent régresser ou même disparaitre simplement par le début d'une miellée subite ou par une récolte massive d'un nouveau pollen sur une plante qui arrive à sa période de floraison. $I_{i}$ e résultat est encore plus net si l'on déplace les colonies d'une miellée à une autre lors de la transhumance. Le changement de flore et de récolte suffit bien souvent à atténuer et même annuler l'effet de la maladie, principalement en ce qui concerne la loque européenne. Je montrerai par la suite dans quelle mesure le facteur alimentaire peut jouer un rôle dans la guérison du couvain malade.

I1 faut attendre $195^{2}$ pour voir des chercheurs s'intéresser à nouveau à la substance bactériostatique du pollen sur des bactéries autres que Bacillus alvei ou Bacillus lavvae. Chauvin, Defromont, Louveaux et VERGé s'aperçoivent au cours d'expériences sur l'action des pollens dans la nutrition des mammifères que les excréments des animaux traités au pollen contiennent peu de bactéries. Si des souris reçoivent un régime standard additionné d'un extrait éthéropétrolique de pollen les résultats 
sont moins nets: il existe une flore bactérienne non négligeable dans les excréta, mais celle-ci est moins abondante que dans les excréments des souris témoins sur aliment standard. Ces auteurs ont trouvé également que l'ensemencement d'Escherichia coli, sur un milieu au pollen entier plus gélose, ne donnait pas de résultat. Leur conclusion est que le pollen renferme une substance retardatrice de la croissance des bactéries de la flore intestinale de la souris. Cette substance est très efficace sur Escherichia coli, au contraire, ils n'ont pas trouvé d'action antibactérienne sur Bacillus alvei.

A la suite de ces essais préliminaires encourageants, j'ai continué ces travaux en collaboration avec Defromon'T (résultats non publiés). Nous avons mis en évidence l'action du pollen sur l'accélération de la sporulation chez Bacillus megatherium et Bacillus subtilis. Avec un pourcentage de I p. roo de pollen dans un milieu liquide après 4 heures le Bacillus megatherium commence à sporuler, tandis que les témoins présentent seulement des formes végétatives. I/aspect $d u$ voile bactérien subit également des modifications macroscopiques. D'autre part, le pollen ne doit pas être épuisé par l'éther avant 1'alcool, car les extraits alcooliques sont alors dépourvus d'activité.

Ces recherches furent reprises pendant plusieurs années par CHAUVIN et LAVIE. publiées en I956 et I958 puis complétées. Nous allons les examiner :

\section{B. - Travaux personnels.}

\section{I ${ }^{0}$ Matériel et méthodes.}

I es échantillons sont prélevés de diverses manières selon qu'il s'agit du pollen récolté sur la plante, des pelotes récoltées par les abeilles ou du pollen stocké dans les rayons. Les pollens pulvérulents sont ramassés à la main soit en secouant les rameaux fleuris sur des écrans disposés pour cet usage, soit en plaçant des rameaux dans des vases au laboratoire, et en récupérant le pollen qui tombe, au cours de leur floraison. I,e pollen en pelote est récolté dans les pièges à pollen à l'entrée des ruches; il en tombe des quantités très appréciables dans un tiroir que l'on retire de temps à autre. Après détermination de l'origine botanique, ce pollen est extrait soit immédiatement soit conservé à différentes températures peirdant un temps donné (notamment pour les essais sur le vieillissement). L,e pollen emmagasiné dans les rayons est récolté cellule par cellule avec une aiguille lancéolée en évitant de le souiller de miel ou de cire. Cette opération est facile, le pollen étant assez souvent groupé dans les mêmes zones de la ruche. On peut également établir le spectre pollinique de ces prélèvements. La technique de conservation est la même que pour le pollen en pelote. 
L'extraction des substances antibiotiques du pollen est pratiquée de différentes manières. J'ai déjà signalé dans la première partie de ce chapitre qu'une extraction par l'éther avant l'alcool ne donnait pas de bons résultats. L'antibiotique passe dans l'éther, mais on extrait en même temps beaucoup de lipides, qui rendent difficile et parfois impossible l'incorporation de l'extrait au milieu de culture. Au contraire, les extraits alcooliques et les extraits aqueux sont d'emploi très facile et permettent d'obtenir des résultats réguliers. Il suffit d'un quart d'heure d'ébullition à reflux pour arracher au pollen la quasi totalité de ses principes anti-

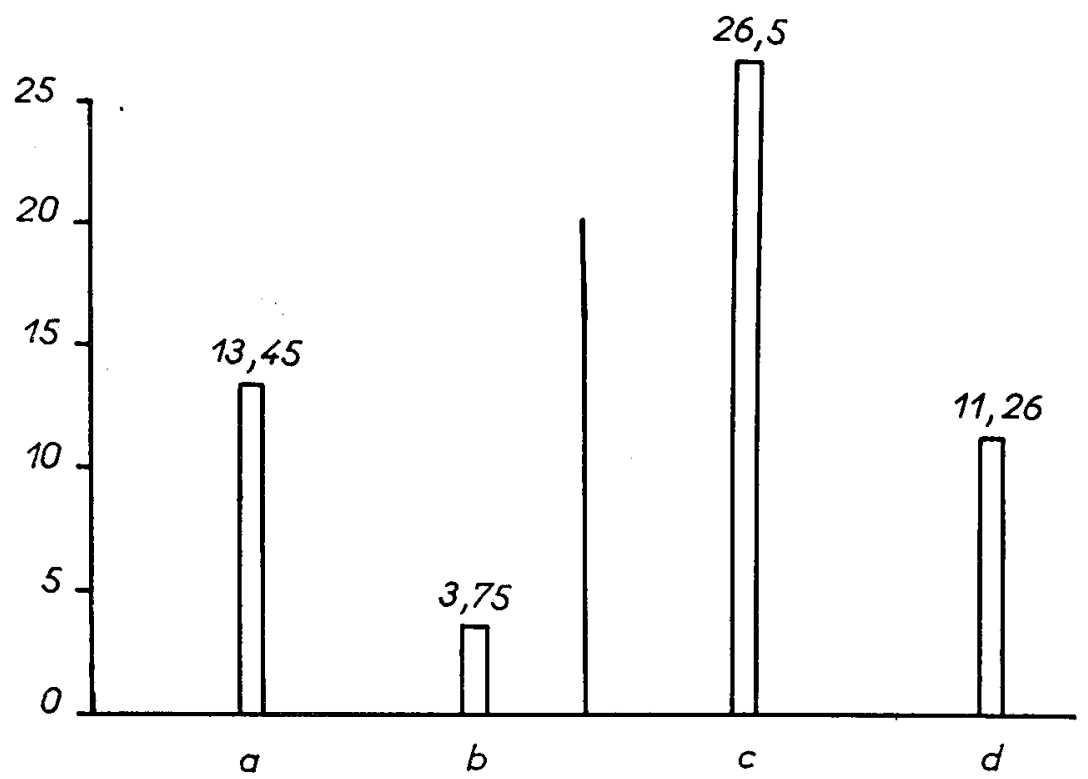

FIg. 25. - Action antibiotique clu pollen récolté par les abeilles (épreuve a et b sur B. subilis et $c$ et d sur B. Proteus rulgaris).

$a$ et $c$ : extrait par l'alcool à chaud.

$b$ et $d$ : extrait par l'alcool à froid.

biotiques. L'extraction à froid est également possible. J'ai d'ailleurs comparé l'extraction alcoolique à froid, par broyage dans un mortier et l'extraction alcoolique à chaud au soxhlet pendant 3 heures. Les résultats consignés sur la figure 25 donnent la différence d'activité de ces det1x extraits de pollen en pelotes sur Bacillus subtilis souche Caron et Proteus vulgaris. Nous pouvons remarquer que l'extrait alcoolique à froid est beaucoup moins efficace que l'extrait alcoolique à chaud. L'extraction par l'eau froide peut être aussi utilisée, mais la macération doit se prolonger douze heures au réfrigérateur. De toute manière, bien que cette dernière méthode soit intéressante dans certains cas (pollen en cellules), l'extraction par l'alcool à chaud est bien préférable. Après filtration au 
Büchner, les liqueurs sont évaporées et reprises par l'eau avant d'être additionnées aux milieux de culture.

Qu'on ait employé pour l'extraction l'alcool ou l'eau, les liqueurs finales en milieu aqueux ont la même apparence et une couleur d'un jaune rougeâtre. Ces substances sont stables et se modifient peu à l'abri de la lumière et au réfrigérateur. Elles sont thermostables et résistent à l'autoclavage à $\mathrm{I} 20^{\circ}$ pendant 30 minutes. Le $\mathrm{pH}$ des extraits est très acide, mais ceux-ci gardent leur valeur antibiotique après neutralisation. A des concentrations trop élevées dans le milieu gélosé, les extraits de pollens empêchent la solidification du milieu et gênent l'interprétation des résultats.

SMITH, BECK et ANDERson avaient extrait comme nous l'avons vu le facteur de sporulation de Bacillus larvae par l'eau. Ils ont montré également que l'extrait éthéré avait la même efficacité et que la substance de sporulation pouvait être enlevée par l'éther de sa solution aqueuse.

En ce qui me concerne, j'ai pratiqué l'épreuve des souches microbiennes par la méthode de la dilution; le calcul des unités a été effectué comme précédemment.

\section{$2^{0}$ Résultats obtenus avec le pollen en pelotes récolté dans les trappes à pollens.}

a) Action antibiotique D'extraits DE POLLENS MÉLANGés D'ORIGINE BOTANIQUE VARIÉE.

Les mélanges de pollens sont toujours actifs sur les 30 souches bactériennes du tableau I. L'action de l'extrait du pollen en pelote vis-àvis de 28 souches est consignée sur le tableau XI. J'ai essayé également 1'antibiotique des pollens sur Bacillus larvae et Bacillus alvei; ces deux bactéries sont inhibées, principalement Bacillus alvei. Nous verrons au chapitre VII ce qu'il faut penser de cette action sur les agents des maladies des abeilles.

Si l'on analyse le tableau XI on remarque aussi l'intense activité en ce qui concerne certaines souches de Proteus et de Salmonella. L'activité est très forte également sur les différentes souches d'Escherichia coli, plus même que le tableau ne la laisserait supposer, car ma méthode d'épreuves n'était pas très précise, puisqu'elle allait seulement du simple au double, et dans le cas des Escherichia les concentrations limites ont été très sévèrement évaluées (1a culture bactérienne était encore très peu développée à la dilution double de la limite prise comme base des calculs dans le cas des Escherichia).

J'ai porté également des résultats sur un graphique (figure 26) pour comparer l'activité de l'extrait du pollen à l'extrait d'abeille sur 
I3 souches bactériennes. Toutes les valeurs sont calculées d'après l'étalon $\mathrm{U}=$ Io pour Bacillus subtilis souche Caron aussi bien pour un extrait que pour 1'autre. On remarque que les deux antibiotiques ont des traits communs, par exemple :

Activité très grande sur Salmonella gallinarum, Proteus vulgaris.

Activité à peu près de la même valeur que sur Bacillus subtilis pour

TABLEAU XI

\begin{tabular}{|c|c|c|c|c|c|c|c|c|}
\hline \multirow[b]{2}{*}{ Souches essayées } & \multicolumn{8}{|c|}{ Action antibiotique de l'extrait pollen } \\
\hline & $2 \mathrm{~cm}^{3}$ & $\mathrm{I} \mathrm{cm}^{3}$ & $0,5 \mathrm{~cm}^{3}$ & $\begin{array}{l}0,25 \\
\mathrm{~cm}^{3}\end{array}$ & $\begin{array}{l}0,15 \\
\mathrm{~cm}^{3}\end{array}$ & $\begin{array}{l}\mathrm{O}, \mathrm{IO} \\
\mathrm{cm}^{3}\end{array}$ & $\begin{array}{l}0,05 \\
\mathrm{~cm}^{3}\end{array}$ & $\begin{array}{l}\text { témoin } \\
2 \mathrm{~cm}^{3} \\
\mathrm{H}_{2} \mathrm{O}\end{array}$ \\
\hline 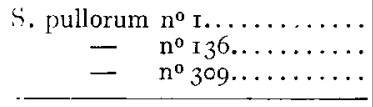 & 二 & 二 & $\overline{-}$ & $\overline{-}$ & $\begin{array}{l}+ \\
+ \\
+ \\
\end{array}$ & $\begin{array}{l}+ \\
+ \\
+\end{array}$ & $\begin{array}{l}+ \\
+ \\
+\end{array}$ & $\begin{array}{l}+ \\
+ \\
+\end{array}$ \\
\hline $\begin{array}{cc}\text { S. Gallinarum } & n^{\mathbf{o}} 38 \ldots \ldots \ldots \ldots \\
- & n^{\mathbf{0}} 47 \ldots \ldots \ldots \ldots \\
- & n^{\mathbf{0}} 237 \ldots \ldots \ldots \\
\end{array}$ & $\bar{z}$ & $\overline{-}$ & $\bar{z}$ & $\begin{array}{l}- \\
\bar{z}\end{array}$ & + & $\begin{array}{l}+ \\
+ \\
+\end{array}$ & $\begin{array}{l}+ \\
+ \\
+\end{array}$ & $\begin{array}{l}+ \\
+ \\
+ \\
\end{array}$ \\
\hline $\begin{array}{cc}\text { S. type Dublin } n^{0} 4-54 \ldots \ldots \\
- & n^{0} 5-54 \ldots \ldots \cdots \\
- & n^{0} 7-54 \ldots \cdots \cdots\end{array}$ & $\bar{z}$ & $\overline{-}$ & + & $\begin{array}{l}+ \\
+ \\
+\end{array}$ & $\begin{array}{l}+ \\
+ \\
+ \\
+\end{array}$ & $\begin{array}{l}+ \\
+ \\
+ \\
\end{array}$ & $\begin{array}{l}+ \\
+ \\
+\end{array}$ & $\begin{array}{l}+ \\
+ \\
+\end{array}$ \\
\hline 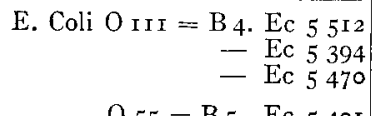 & $\overline{-}$ & $\overline{-}$ & $\begin{array}{l}+ \\
+ \\
+ \\
+\end{array}$ & $\begin{array}{l}+ \\
\dot{+} \\
+\end{array}$ & $\begin{array}{l}+ \\
+ \\
+\end{array}$ & $\begin{array}{l}+ \\
+ \\
+\end{array}$ & $\begin{array}{l}+ \\
+ \\
+\end{array}$ & t- \\
\hline $\begin{aligned} \mathrm{O}_{55}= & \text { B } 5 . \\
& -\mathrm{Ec} 540 \mathrm{I} \\
& -\mathrm{Ec} 5423 \\
& \text { Ec } 543^{8}\end{aligned}$ & $\overline{-}$ & $\overline{-}$ & $\begin{array}{l}+ \\
+ \\
+\end{array}$ & $\begin{array}{l}+ \\
+ \\
+\end{array}$ & $\begin{array}{l}+ \\
+ \\
+\end{array}$ & $\begin{array}{l}+ \\
+ \\
+\end{array}$ & $\begin{array}{l}+ \\
+ \\
+\end{array}$ & $\begin{array}{l}+ \\
+ \\
+\end{array}$ \\
\hline 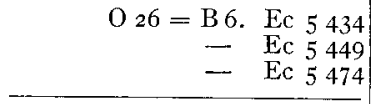 & $\overline{-}$ & 二 & $\begin{array}{l}+ \\
+ \\
+\end{array}$ & $\begin{array}{l}+ \\
+ \\
+\end{array}$ & $\begin{array}{l}+ \\
+ \\
+\end{array}$ & $\begin{array}{l}+ \\
+ \\
+\end{array}$ & $\begin{array}{l}+ \\
+ \\
+\end{array}$ & $\begin{array}{l}+ \\
+ \\
+\end{array}$ \\
\hline 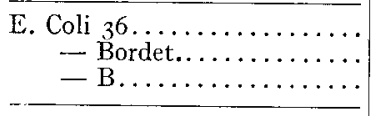 & $\bar{z}$ & 二 & + & $\begin{array}{l}+ \\
+ \\
+\end{array}$ & $\begin{array}{l}+ \\
+ \\
+\end{array}$ & $\begin{array}{l}+ \\
+ \\
+ \\
+\end{array}$ & $\begin{array}{l}+\dot{+} \\
+ \\
+\end{array}$ & $\begin{array}{l}+ \\
+ \\
+ \\
+\end{array}$ \\
\hline 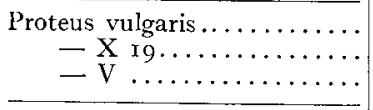 & $\overline{-}$ & $\overline{-}$ & $\begin{array}{l}- \\
- \\
-\end{array}$ & $\overline{-}$ & 二 & \pm & $\begin{array}{l}+ \\
+ \\
+\end{array}$ & $\begin{array}{l}+ \\
+ \\
+\end{array}$ \\
\hline B. Subtilis souche Caron...... & - & - & $-\because$ & + & + & + & + & + \\
\hline 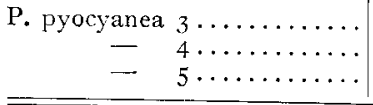 & $\bar{E}$ & $\overline{-}$ & + & $\begin{array}{l}+ \\
+ \\
+\end{array}$ & $\begin{array}{l}+ \\
+ \\
+\end{array}$ & $\begin{array}{l}+ \\
+ \\
+\end{array}$ & $\begin{array}{l}+ \\
+ \\
+\end{array}$ & $\begin{array}{l}+ \\
+ \\
+\end{array}$ \\
\hline
\end{tabular}

En ce qui concerne l'action antibiotique de l'extrait de pollen, le développement bactérien est représenté par le signe + et l'inhibition par le signe - Les deux signes juxtaposés signifient un développement très lent et presque nul. Chaque colonne verticale correspond à une quantité d'extrait (en centimètres cubes) ajoutée au milieu $\left(\mathrm{Io}_{\mathrm{cm}} \mathrm{cm}^{3}\right)$ le milieu est simplement de l'eau peptonée (peptone "Rhône Poulenc ") additionnée de gélose. Les résultats des tests sont nuls ou inversés avec diverses peptones (peptone Merck par exemple). $100 \mathrm{~cm}^{3}$ de solution correspondent à I 00 unités subtilis.

Remarques:

Les 6 premières souches ( $S$. pullorum et $S$.gallinarum) ont un développement lent, même les témoins. En ce qui concerne $E$. coli, avec $0,5 \mathrm{~cm}^{3}$ d'extrait, il y a très peu de développement (sauf Ec 5470 et Ec 5423). B. subtilis souche Caron nous a fourni les mêmes résultats, que l'extrait de pollen soit autoclavé ou non avant l'ensemencement. 
les souches: Salmonella type Dublin, plusieurs Escherichia, Pseudomonas pyocyanea et Bacillus larvae.

Des différences notables sur les bactéries suivantes : Escherichia coli Bordet et Bacillus alvei.

En conclusion, je puis dire que les deux antibiotiques sont actifs sur les mêmes souches bactériennes (3o souches), mais très différents du point de vue quantitatif. Ils se ressemblent beaucoup par leur grande activité sur Salmonella gallinarum $n^{0} 38$ et sur Proteus vulgaris. Cependant, l'essai sur Bacillus alvei montre une inversion des courbes, l'extrait de pollen étant deux fois moins actif que celui de l'abeille. Il ne s'agit donc pas de la même substance antibiotique ou du même mélange d'antibiotiques.

D'autre part, si l'on considère l'activité de l'extrait de pollen et ceux de la propolis et de la cire ou du miel, il apparaît de très grandes différences et l'antibiotique du pollen est de loin le plus intéressant sur de nombreuses souches bactériennes (voir figure 27).

J'ai également exécuté des essais par les méthodes plus classiques de la diffusion, à l'aide de disques imbibés d'antibiotique et déposés sur des plaques de gélose ensemencées. Les résultats sont assez satisfaisants et les auréoles d'inhibition nettes. Je n'ai pas toujours appliqué cette technique, car bien souvent, et principalement au début de mes recherches mes liqueurs d'extraction insuffisamment purifiées étaient souillées de dérivés résineux ou lipidiques qui gênaient la diffusion dans le milieu et l'interprétation des résultats.

b) ACTION ANTIBIOTIQUE D'EXT'TRATS DE POLLENS D'UNE SEULE ESPÈCE.

Je viens de montrer qu'un mélange de divers pollens est toujours actif dans les épreuves auxquelles je les ai soumis. Nous allons voir qu'il n'en est pas de même si l'on trie les pelotes de pollen et que l'on essaye les pollens de plantes variées séparément; ceux-ci manifestent une action antibiotique bien différente, de l'un à l'autre. Les essais ont porté sur I3 pollens récoltés par les abeilles. 20 grammes de pollens purs étaient extraits par $50 \mathrm{~cm}^{3} \mathrm{~d}$ 'alcool absolu à ébullition à reflux pendant $\mathrm{I} / 4 \mathrm{~d}$ 'heure L,e tout est ensuite filtré au Büchner, repris par $50 \mathrm{~cm}^{3}$ d'alcool absolu bouillant, filtré à nouveau, évaporé au bain-marie et repris par ro $\mathrm{cm}^{3}$ d'eau. L'extrait en milieu aqueux est essayé par la méthode de la dilution.

Les résultats obtenus sont reportés dans le tableau XII. Les extraits de pollens n'ont pas tous la même activité ; certains sont très riches en substances antibiotiques, par exemple le pollen de maïs, de châtaignier, de pissentit, de trèfle incarnat. D'autres pollens ont une activité moyenne ou faible tels que le pollen de ciste et d'Erica arborea. Enfin, certains en sont dépourvus totalement: colza, prumus (mélanges divers), lierre, coquelicot. 


\section{TABLEAU XII}

Action antibiotique de différents pollens en pelotes sur Bacillus subtilis.

\begin{tabular}{|c|c|c|c|}
\hline Ispèce botanique & pII de l'extrait & $\begin{array}{l}\text { Résidu sec pour } \\
\text { I g. de pollen extrait }\end{array}$ & Unités antibiotiques \\
\hline 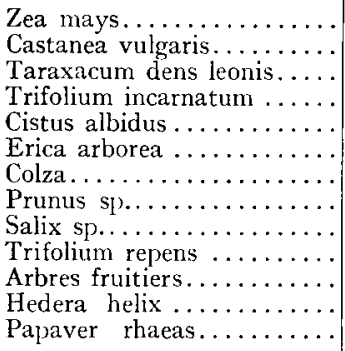 & $\begin{array}{l}2,5 \\
3 \\
4 \\
5 \\
5 \\
5 \\
4 \\
5 \\
5,5 \\
3,5 \\
5 \\
5 \\
5\end{array}$ & $\begin{array}{l}0,135 \\
0,214 \\
0,240 \\
0,188 \\
0,235 \\
0,759\end{array}$ & $\begin{array}{c}\mathbf{I}, 85 \\
\mathbf{I}, \mathbf{I} \\
\mathbf{I} \\
0,9 \\
0, \mathbf{I} \\
0,06 \\
\\
\\
\text { Pas d'action } \\
\text { antibiotique }\end{array}$ \\
\hline
\end{tabular}

Que faut-il déduire de ces résultats?

Tout d'abord dans le pollen en mélange trouvé dans les trappes placées sur les ruches, il existe toujours une substance antibiotique. On peut supposer que les abeilles font un choix dans leur récolte. L,OUvEAuX (I958) a montré que ce choix existait en ce qui concerne la valeur alimentaire et que les ouvrières récoltaient en fonction des besoins physiologiques de la colonie. Je pense que ce choix existe également en ce qui concerne la valeur antibiotique des pollens mais peut-être est-ce simplement un résultat statistique tenant à ce que les plantes à antibiotique sont en moyenne assez fréquentes.

I1 est difficile d'attribuer au pH des extraits leur action antibactérienne. En effet, le tableau XII nous montre qu'il n'y a pas de relation simple entre ces deux facteurs. Par exemple, l'extrait de trèfle incarnat, $\mathrm{pH}-5$ est antibiotique, alors que le trèfle blanc, $\mathrm{pH}-3,5$ ne l'est pas. D'autre part, la neutralisation des liqueurs n'amène pas de différence dans les résultats obtenus. Nous ne trouvons pas de relation non plus suivant qu'il s'agit d'une famille botanique ou d'une autre, de plantes arbustives ou de plantes herbacées, de floraisons printanières ou plus tardives. Il n'y a pas non plus de rapport entre le poids du résidu sec et brut obtenu pour I gramme de pollen et le nombre d'unités antibiotiques présentes. Par exemple, le résidu sec obtenu avec le pollen d'Erica arborea est très important (les $3 / 4$ du poids initial) et l'activité antibiotique est extrêmement faible.

Actuellement, je tends à penser que les substances antibiotiques sont voisines dans les divers pollens et que nous avons affaire simplement à des différences quantitatives. 
c) CoMparaison ENTRE LA VALEUR ANTIBIOTIQUe DU POLLEN RÉCOLTÉ PAR LES ABEILLES ET DU POLLEN RÉCOLTÉ A LA MAIN.

La substance antibiotique vient-elle des abeilles? C'est la question que je me suis posée après les résultats obtenus avec les pollens récoltés dans les tiroirs des pièges à pollens. En effet, nous avons vu dans les généralités que les abeilles lors de la récolte du pollen en agglutinent les grains à l'aide d'une régurgitation gastrique, et l'on n'ignore pas qu'elles y ajoutent ainsi diverses substances, des sucres et des diastases en particulier. On peut se demander alors si les principes antibiotiques ne proviennent pas, en tout ou en partie, des ouvrières et non du pollen. A vrai dire, une telle hypothèse est rendue improbable du fait des très grandes différences de

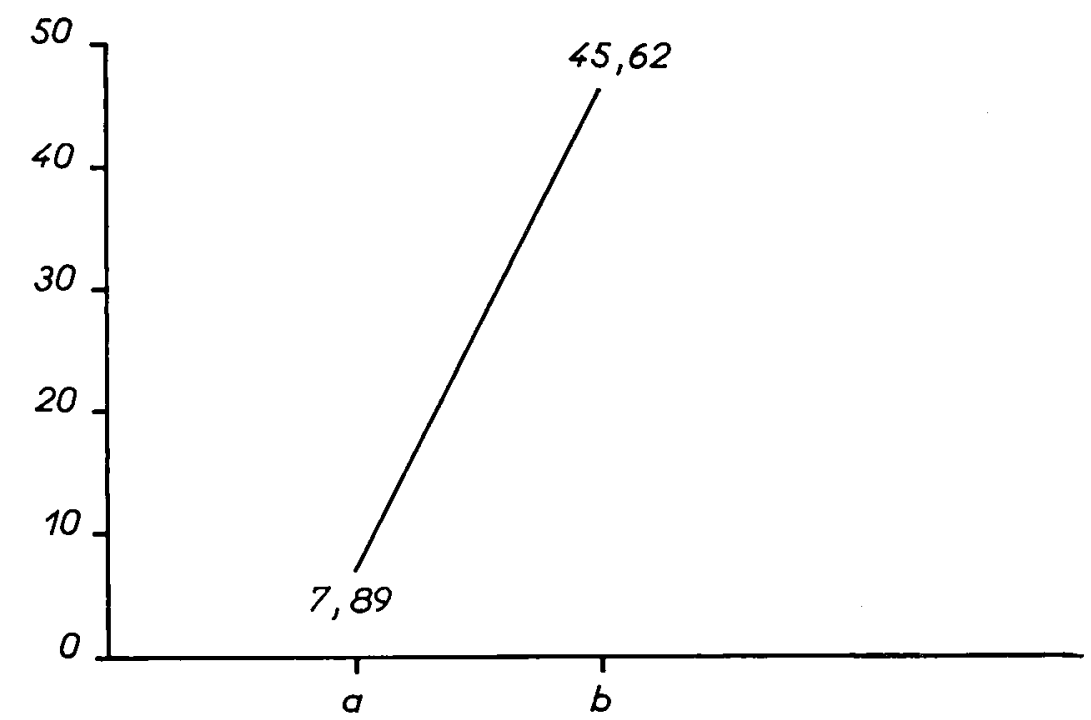

FIG. 28. - Compraraison de la valeur antibiotique entre un pollen récolté à la main et un pollen récolté par les abeilles (épreuve B. subtilis).

$a:$ pollen de maïs récolté ì la main.

$b:$ pollen de maïs récolté par les abeilles.

pouvoir antibiotique des différents pollens en pelotes. Il faudrait supposer, fort arbitrairement, que les abeilles régurgitent plus ou moins de substance antibiotique suivant l'espèce de pollen ou même n'en régurgitent pas du tout, puisque certains sont privés de tout pouvoir antibiotique. Mais il est possible de récolter certains pollens en quantité suffisante sur les plantes elles-mêmes, sans l'aide des abeilles. C'est le cas des pollens de conifères, de Corylus avellanea et églement de Zea Mays. J'ai comparé le pollen de Corylus récolté à la main et celui qui est récolté par les abeilles; ce dernier est plus actif que le pollen récolté à la main, mais je ne dispose pas de mesures précises. Au contraire, j'ai étudié d'une manière approfondie le pollen de maïs. Or le pollen récolté à la main contient moins 
d'unités antibiotiques que le pollen des trappes. I a figure 28 montre la différence dans l'épreuve sur Bacillus subtilis, dans ce cas l'action du pollen récolté à la main est 5 à 6 fois plus faible que l'action antibiotique du pollen en pelotes. TODD et BRETHERICK (I942), ont montré également que le pollen des trappes était toujours plus actif biologiquement sur l'abeille que le pollen récolté à la main. Je pense donc que si le pollen recèle des antibiotiques, ceux-ci doivent être complétés par l'action des substances que l'abeille y ajoute et il est possible également qu'ils y soient modifiés ou démasqués.

\section{$3^{0}$ Résultats obtenus avec les extraits de pollen emmagasinés dans les cellules.}

\section{a) Action DU POLLEN STOCKÉ.}

Le pollen stocké dans les rayons à l'intérieur de la ruche et prélevé comme je l'ai expliqué plus haut donne des extraits qui sont toujours plus actifs (même neutralisés) que le pollen en pelote venant d'être récolté

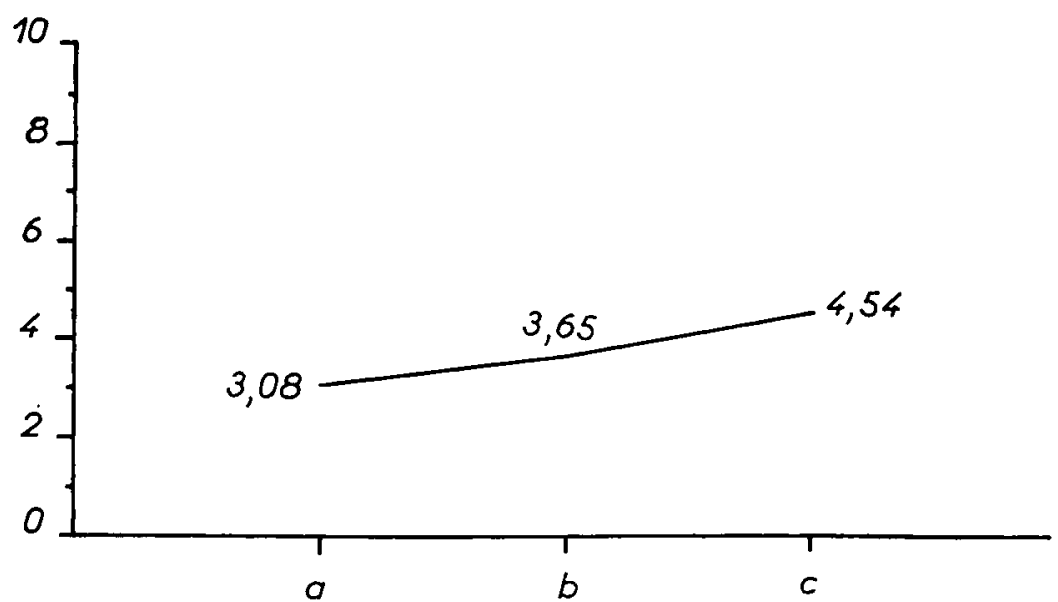

FIG. 20. - Maturation du pollen et valeur antibiotique de ces extraits (épreuve $B$. sublilis). $a:$ pollen en pelotes.

$b$ : pollen en cellules mais frais.

$c$ : pollen en collules depuis I an.

Ces résultats ont été vérifiés sur un grand nombre de souches bactériennes. L'activité du pollen d'une même ruche (et à l'intérieur de celle-ci) non seulement se maintient, mais s'accroît en fonction du temps. La figure 29 résume les mesures en ce qui concerne l'épreuve sur Bacillus subtilis. (Il s'agit là de pollens variés et non d'un pollen bien déterminé.) Or nous savons d'après Svoboda (I940) et HirchCoOck (I956) que le pollen stocké dans les cellules subit une fermentation lactique. Cependant nous sommes, encore mal renseignés sur l'importance et le processus de cette fermenta- 
tion. Il se produit également dans le pollen des phénomènes d'hydrolyse. Enfin, nous avons déjà vu que le pollen stocké dans la ruche perd son pouvoir germinatif. On peut alors admettre que l'addition de plusieurs substances en quantité variable ou les fermentations renforcent la valeur antibactérienne des pollens. Il faut noter aussi que WHITE (Igo6) a isolé de l'intestin de l'abeille et du pollen de rayons une même bactérie le Bacillus " $B$ " que l'on trouve fréquemment et qui participe peut-être aux fermentations.

b) Comparaison de l’activité dU POLLEN STOcké et DU POLLEN EN PELOTES (SUR UNE SEULE ESPÈCE DE POLLEN).

J'ai comparé 1'action d'un même pollen, celui de Castanea vulgaris, suivant qu'il est récolté dans les trappes à pollen et frais ou qu'il est stocké dans la ruche depuis une huitaine de jours. J'ai choisi ce pollen à

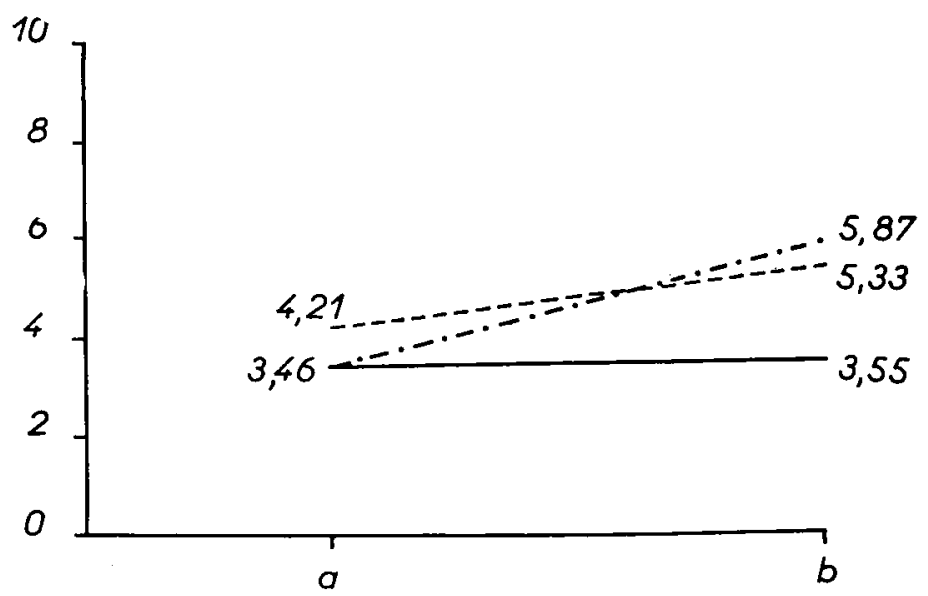

Fir. 30. - Comparaison de la valeur de deux extraits de pollens de Castanea anlgaris récoltés par ( $a$, pollen en pelotes et $b$, pollen stocké dans les cellules).

- B. subtilis Caron.

-.-.-. Proteus rulgaris. -... B. aloei.

cause de son action antibiotique assez nette, de la possibilité de le récolter en quantité suffisante et de sa détermination facile. Il a été recueilli sur la même ruche au moment de la floraison du châtaignier. J'ai essayé les extraits alcooliques de ces échantillons sur trois souches bactériennes, Bacillus subtilis, Proteus vulgaris, et Bacillus alvei. La figure 30 montre l'activité des deux extraits sur ces bactéries. On remarque que les substances antibiotiques sont toujours plus actives dans le pollen stocké à l'intérieur de la ruche bien que celui-ci ne soit entreposé que depuis quelques jours. La différence est particulièrement très nette pour Proteus vulgaris. En ce qui concerne Bacillus subtilis, les chiffres sont pratique- 
ment les mêmes, mais dans d'autres expériences répétées avec des pollens en mélange, j'ai souvent trouvé des valeurs supérieures pour les pollens stockés et jamais celles-ci n'ont été inférieures à la valeur du pollen des trappes.

Il est donc certain que le pollen de la plante, puis récolté par l'abeille et enfin ensilé dans la ruche montre une activité antibiotique de plus en plus marquée.

\section{$4^{\circ}$ Vieillissement et évolution des substances antibiotiques présentes dans les pollens récoltés par l'abeille et conservés à différentes températures au laboratoire.}

\section{a) Conservation a température ORdinaire.}

L'extraction d'un lot de pollens variés récoltés à la trappe à pollen et conservé 9 mois sans précautions spéciales et à la température du laboratoire à Montfavet m'a montré que l'antibiotique obtenu était plus actif, et de loin, que la moyenne des substances présentes dans les pollens en pelote venant d'être récoltés. Des expériences complémentaires ont toujours confirmé ces résultats, l'antibiotique est de plus en plus actif au cours du vieillissement du pollen à température ordinaire.

\section{b) Conservation aU froid.}

Après toute une série d'expériences préliminaires, j'ai voulu savoir si les facteurs antibiotiques présents dans les pollens se modifiaient encore à des températures assez basses.

J'ai choisi pour ces essais le pollen de Castanea vulgaris d'une part en pelote et de l'autre stocké en rayons.

Io Du pollen de châtaignier est prélevé dans des trappes disposées à l'entrée de la ruche dès que les abeilles viennent de l'abandonner. Ce pollen est immédiatement placé et conservé $\dot{a}-35^{\circ} \mathrm{C}$, afin de bloquer toutes les transformations biochimiques possibles. Si l'on soumet ce pollen à l'extraction alcoolique (même 4 à 5 mois plus tard) en vue de mesurer son pouvoir antibiotique sur Bacillus subtilis, et Proteus vulgaris, on s'aperçoit qu'il ne contient que peu d'Unités actives ( 1,68 pour subtilis et 2,52 pour Proteus). Ce pollen n'a pratiquement pas évolué à $-35^{\circ} \mathrm{C}$. Le tableau XIII montre ces résultats et ceux obtenus avec le même pollen conservé ensuite 20 jours et 60 jours à $+I^{\circ} \mathrm{C}$. Ces expériences m'ont permis de constater que même à $\mathrm{o}^{\circ}$ le pollen en pelote évolue et que son facteur antibiotique augmente d'activité en fonction du temps.

$2^{\circ}$ Du pollen de châtaignier est prélevé dans les rayons de la même ruche que précédemment, c'est-à-dire qu'il est stocké dans les cellules depuis quelques jours. Ce pollen est immédiatement placé et conservé à $-35^{\circ} \mathrm{C}$ au réfrigérateur (comme pour le pollen en pelote et pendant 4 à 
TABLEAU XIII

Étude du vieillissement au laboratoire du pollen de châtaignier en pelotes.

\begin{tabular}{|c|c|c|c|}
\hline Extraits (date d'extraction) & Souches éprouvées & $U$ & U par $g$ de pollen \\
\hline $\begin{array}{l}\left.1^{\circ}\right) \text { le } 20 / \text { I } 1 / 5^{8} \\
\text { à la sortie du réfrigérateur à }-35^{\circ} \mathrm{C} \text {. }\end{array}$ & $\begin{array}{l}\text { Bacillus subtilis souche Caron } \\
\text { Proteus rulgaris }\end{array}$ & $\begin{array}{l}1,68 \\
2,52\end{array}$ & $\begin{array}{l}0,33 \\
0,50\end{array}$ \\
\hline $\begin{array}{l}2^{\circ} \text { ) le } \mathrm{IO} / \mathrm{I} 2 / 5^{8} \\
20 \text { jours après (conservé à } \\
\mathrm{I}^{\circ} \mathrm{C} \text { ). }\end{array}$ & $\begin{array}{l}\text { Bacillus subtilis souche Caron } \\
\text { Protens inlgaris }\end{array}$ & $\begin{array}{l}3,03 \\
6,06\end{array}$ & $\begin{array}{l}0,66 \\
1,32\end{array}$ \\
\hline $\begin{array}{l}\left.3^{\circ}\right) \mathrm{le} 20 / \mathrm{I} / 59 \\
60 \text { jours après (conservé à } \perp \mathrm{I}^{\circ} \mathrm{C} \text { ). }\end{array}$ & $\begin{array}{l}\text { Bacillus subtilis souche Caron } \\
\text { Proteus rulgaris }\end{array}$ & $\begin{array}{l}5,80 \\
8,70\end{array}$ & $\begin{array}{l}1,38 \\
2,08^{3}\end{array}$ \\
\hline
\end{tabular}

5 mois). Le tableau XIV donne les résultats obtenus sous le rapport de l'activité antibiotique selon que ce pollen vient de sortir du réfrigérateur à $-35^{\circ} \mathrm{C}$ ou qu'il a séjourné ensuite 20 à 60 jours à $0^{\circ} \mathrm{C}$. On s'aperçoit que l'activité antibiotique du pollen stocké crô̂t en fonction du temps, même à $\mathrm{o}^{\circ}$, comme pour le pollen en pelote, mais moins rapidement que celui-ci. D'autre part, dès la sortie du réfrigérateur à $-35^{\circ}$, le pollen en rayon est déjà beaucoup plus actif que le pollen en pelote placé dans les mêmes conditions (tableaux XIII et XIV). Ce dernier fait est sans doute dû à la maturation préalable subie par le pollen en rayons pendant les quelques jours où il a séjourné dans la ruche avant sa conservation à $-35^{\circ}$.

\section{TABLEAU XIV}

Étude du vieillissement au laboratoire du pollen de châtaignier stockéen rayons

\begin{tabular}{|c|c|c|c|}
\hline $\begin{array}{l}\text { Extraits } \\
\text { (date d'extraction) }\end{array}$ & Souches éprouvées & $\mathrm{U}$ & $\mid \begin{array}{l}\mathrm{U} \\
\operatorname{par} g \text { de pollen }\end{array}$ \\
\hline $\begin{array}{l}\mathrm{I}^{\circ} \text { le } 20 / \mathrm{II} / 58 \text { à la sortie du réfri- } \\
\text { gérateur à }-35^{\circ} \mathrm{C} \text {. }\end{array}$ & $\begin{array}{l}\text { Bacillus subtilis souche Caron } \\
\text { Protcus mulgaris }\end{array}$ & $\begin{array}{l}2,92 \\
5,84\end{array}$ & $\begin{array}{l}0,714 \\
1,43\end{array}$ \\
\hline $\begin{array}{l}2^{\circ} \text { le } 10 / \mathrm{I}_{2} / 5^{8} \text { 20 jours après (con- } \\
\text { servé à } \pm \mathrm{I}^{\circ} \mathrm{C} \text { ). }\end{array}$ & $\begin{array}{l}\text { Bacillus subtilis souche Caron } \\
\text { Proteus volguris }\end{array}$ & $\begin{array}{l}4,25 \\
5,67 \\
\end{array}$ & $\begin{array}{l}1,0,3 \\
I, 36\end{array}$ \\
\hline $\begin{array}{l}3^{\circ} \text { le } 10 / 1 / 5960 \text { jours après (con- } \\
\text { servé a } \pm 1^{\circ} \mathrm{C} \text { ). }\end{array}$ & $\begin{array}{l}\text { Bacillus subtitis souche Caron } \\
\text { Proteus vnlgaris }\end{array}$ & $\begin{array}{l}4,+0 \\
8,80\end{array}$ & $\begin{array}{l}1,36 \\
2,7^{2}\end{array}$ \\
\hline
\end{tabular}

\section{c) Conclusion.}

L'augmentation de la valeur antibiotique s'observe pendant plusieurs mois dans la ruche ou au laboratoire dans des conditions artificielles. Mais le processus est fonction de la température et s'accélère quand elle augmente. Il est possible, aux environs de $-35^{\circ} \mathrm{C}$, d'empêcher l'évolution et le vieillissement des pollens.

Cet ensemble de changements qui intervient dans la conservation du pollen dans la ruche est certainement dû, comme nous l'avons déjà 
exposé ci-dessus, à des transformations biochimiques telles que, hydrolyse, oxydation, fermentation, etc. Il est possible que cette augmentation soit due en partie à l'accroissement considérable et bien connu du taux d'acide lactique dans les pollens au cours du stockage, surtout s'ils ont été conservés dans les rayons. D'ailleurs, au point de vue de l'activité sur les ovaires des ouvrières, PAIN (1959) a montré que des pollens récoltés et non stockés dans les rayons n'ont pas la même action que ceux qui ont été emmagasinés dans les cellules à l'intérieur de la ruche.

On peut admettre également, dans certains cas, l'action de champignons qui se développent dans les cellules à pollen et peuvent modifier la valeur antibiotique du produit.

\section{$5^{\circ}$ Fractionnement de la substance antibiotique du pollen.}

Les extraits alcooliques repris par l'eau ont tout d'abord été centrifugés à 2200 tours minute pendant ro minutes pour éliminer le précipité. Il se trouve que le culot de centrifugation repris par l'eau ou l'alcool est totalement inactif. D'autre part, l'extrait filtré, mais non centrifugé et l'extrait après centrifugation renferment le même nombre d'unités sur Bacillus subtilis. Partant de ce principe, nous avons adopté la méthode suivante pour fractionner la substance active dans les pollens.

Lorsqu'on veut purifier l'extrait alcoolique, il est bon de laisser la solution, filtrée au Büchner à la glacière pendant vingt-quatre heures. Il se dépose alors un abondant précipité inactif. La solution est concentrée à sec au bain-marie et reprise par l'eau bouillante ; une grande partie passe en solution ou en suspension; la solution a une couleur blanc jaunâtre trouble et s'éclaircit après quelques jours de glacière en laissant déposer un précipité inactif ; si l'on est parti d'une extraction par l'eau bouillante au lieu d'alcool, on peut ajouter à l'eau huit fois le volume d'acétone, ce qui permet de séparer un abondant précipité inactif : la solution concentrée à sec, est reprise par l'alcool bouillant, l'alcool évaporé et le résidu repris par l'eau bouillante.

On peut leur faire subir une purification ultérieure en ajoutant de l'extrait de Saturne jusqu'à cessation du précipité. Les principes actifs restent en solution et, après avoir chassé le plomb en excès par l'acide st1lfhydrique, on obtient une liqueur jaune foncé très active. Cette liqueur, chromatographiée sur alumine, fournit une zone jaune foncé éluable par l'ammoniaque, avec au-dessous une zone jaune en contiguité immédiate et qui descend rapidement quand on ajoute de l'eau sur la colonne. Mais les liqueurs chronatographiées sont inactives, et l'alumine semble détruire ou absorber de manière durable le principe actif. Nous n'avons pu trouver jusqu'ici de matière adsorbante convenable qui fixe le corps antibiotique sans l'altérer. Alors que les solutions initiales sont fortement acides, les extraits purifiés sont neutres. 


\section{C. - Conclusion du chapitre III.}

\section{$I^{\circ}$ Comparaison avec les résultats d'autres auteurs.}

L,es travaux concernant l'antibiotique présent dans le pollen récolté par les abeilles sont peu nombreux et nous les avons déjà analysés. En résumé on peut dire qu'on y voit peu de divergences avec mes résultats. Tout d'abord le facteur de sporulation, thermostable a été trouvé actif sur Bacillus lavjae (SMiTH, BECK et ANDERSON) mais également sur Bacillus alvei. De même, j'ai confirmé in vitro l'action antibiotique in vivo chez la souris (Chauvin, Defromont, Louveaux, Vergé) sur Bacillus subtilis, Escherichia coli. J'ai contrôlé comme tous cesaut eurs que l'extraction éthérée de pollens avant l'entraînement par 1'alcool donne des résultats négatifs. D'autre part, le pollen n'a pas de propriété antifongique et un milieu gélosé additionné de pollen entier permet le développement des levures et des moisissures. Mais, en ce qui concerne la valeur antibiotique du pollen sur Bacillus alvei, j'ai toujours trouvé à l'inverse des précédents auteurs une activité antibactérienne très nette par la méthode de la dilution. Je suppose que ces derniers ont pratiqué les épreuves sur des fractions éthéropétroliques; or, nous venons de voir que cette méthode d'extraction n'est pas très bonne et donne des résultats très inégaux.

Chauvin (I957) a étudié un facteur de croissance vis-à-vis des jeunes mammifères, contenu dans les pollens récoltés par les abeilles. J'avais pensé retrouver certaines analogies entre le facteur antibiotique et le facteur de croissance chez la souris blanche; mais l'examen détaillé de ces travaux ne permet pas de rapprocher l'un à l'autre. Le tableau XV montre côte à côte certains résultats obtenus sur des pollens d'espèces

\section{TableaU XV}

Comparaison de l'activité antibiotique et du facteur de croissance chez des pollens récoltés par les abeilles.

\begin{tabular}{|c|c|c|}
\hline & & Facteur de croissance \\
\hline Espece botanique & $\begin{array}{l}\text { Unité antibiotique } \\
\text { (sur Bacillas subtilis) }\end{array}$ & $\begin{array}{l}\text { Avantage par rapport au Témoin en } \% \\
\text { du poids de départ pour une concentration } \\
\text { exprimée en pollen de } 25 \%\end{array}$ \\
\hline 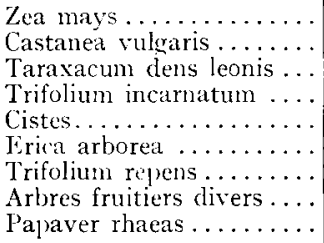 & $\begin{array}{l}\mathrm{r}, 85 \\
\mathrm{I}, \mathrm{I} \\
\mathrm{I} \\
0,9 \\
0, \mathrm{I} \\
0,06 \\
0 \\
0 \\
0\end{array}$ & $\begin{array}{l}0 \\
7.5 \% \\
0 \\
0 \\
12 \% \\
15 \% \\
00 \\
22 \% \\
64 \%\end{array}$ \\
\hline
\end{tabular}


définies. On peut se rendre compte que les pollens contenant le plus d'unités antibiotiques ne sont pas obligatoirement ceux qui renferment la substance de croissance. On remarque par exemple que les pollens d'arbres fruitiers et de coquelicot contiennent le facteur de croissance en grande quantité. Ils ne sont pas antibiotiques à l'égard de Bacillus subtilis; au contraire, les pollens de châtaignier et de maïs, riches en substances antibiotiques, n'ont qu'une action moyenne sur la croissance de la souris. L'on voit qu'il existe de grandes différences d'un pollen à un autre. Dans une même famille, deux plantes peuvent différer totalement, de même que des espèces très éloignées peuvent avoir des valeurs antibiotiques voisines. En résumé, le principe antibiotique du pollen récolté par les abeilles paraît différer du principe accélérateur de la croissance étudié par Chauvin jusqu'à plus ample informé.

Je noterai aussi après CHAuvin, l'action régulatrice du pollen dans les fonctions intestinales humaines, car cette action est peut être en rapport direct ou indirect avec le principe antibiotique que j'ai étudié. Cette activité du pollen se manifeste par un arrêt rapide des diarrhées persistantes d'origine basse, même résistantes aux antibiotiques courants et également par l'amélioration des constipations chroniques et l'action sur certaines colibacilloses. I, pollen pur, dans les cas de constipation avec anomalie de la flore colique, a donné de très bons résultats et toujours meilleurs que ceux obtenus au moyen d'extraits de pollen. Tous les pollens ne sont pas actifs au même degré et certains n'ont pas d'action. Malgré ces résultats et ceux qui ont été obtenus sur les souris nourries au pollen, je ne puis attribuer encore (ou attribuer exclusivement) à l'antibiotique 1'action si nette sur 1'intestin. Des expériences nombreuses sont encore nécessaires pour vérifier ces hypothèses; cependant, il semble qu'il $\mathrm{y}$ ait là une direction intéressante à suivre, car il ne faut pas oublier l'activité très grande du pollen sur les Proteus, bacilles, particulièrement difficiles à combattre dans certaines affections.

\section{$2^{\circ}$ Valeur et signification de l'antibiotique du pollen récolté par l'abeille.}

L'action du pollen sur les bactéries pathogènes de la ruche est très importante. Déjà en I920 Sturtevant affirmait que, dans le cas de la loque européenne, l'influence des conditions extérieures et notamment celle de la nutrition était primordiale. D'après lui, l'apport de pollens frais, en grande quantité, dans la colonie empêche parfois l'extension de la maladie. Depuis quelques années, il est bien établi que non seulement un apport considérable de nourriture extérieure enraye bien souvent l'épizootie, mais qu'un déplacement des ruches d'une miellée à une autre arrête parfois 1'infection. C'est peut-être une différence qualitative de la 
récolte qui entre en jeu et non pas uniquement un apport quantitatif comme l'ont pensé White et Sturtevan's. Si l'on croit SturTevant, c'est le pollen frais qui serait uniquement inhibiteur de Bacillus aliei, or j'ai démontré que le pollen stocké 20 jours voyait son activité antibiotique sur Bacillus alvei passer de 4,2I à ro,86. En ce qui concerne l'action sur Bacillus larvae, agent pathogène de la loque américaine, les extraits de pollens sont deux fois moins actifs que sur Bacillus alvei. Nous avons déjà vu que le pollen contenait un facteur de sporulation envers Bacillus larvae (SMITH, BECK et ANDERSON). J'ai montré que les extraits alcooliques de pollen possédaient un effet retardateur sur le développement de Bacillus larvae et Bacillus alvei lorsqu'ils sont ajoutés au milieu de culture en quantité inférieure à la dilution limite nécessaire à l'inhibition. Cette propriété n'existe pas vis-à-vis de Bacillus alvei chez les autres antibiotiques de la ruche. I,e pollen doit donc avoir une grande importance dans la lutte contre les maladies du couvain, peut-être grâce à sa valeur nutritive, mais certainement encore plus par son principe antibiotique actif sur Bacillus alvei, et Bacillus larvae.

Lors de son vieillissement le pollen subit des transformations. Certains facteurs, d'après MAURrzIo, sont très labiles (substances actives sur le développement des glandes et des ovaires des ouvrières) et peuvent disparaître très facilement. La substance de croissance trouvée dans le pollen (Chauvis) est deux à trois fois plus active dans le pollen frais que dans le pollen desséché sans précaution. Le pollen récolté par l'abeille et emmagasiné plus de 5 jours dans la ruche est incapable de germer. Cette action antigerminative, étudiée par Maurizio, peut être due à plusieurs causes (voir plus haut), mais le pollen après certaines transformations biochimiques (fermentation etc.) peut devenir de luimême incapable de germer. Nous avons vu qu'il subit aussi une fermentation lactique, signe de transformations encore incomplètement connues. 'Tous les auteurs sont également d'accord sur le fait que les pollens récoltés sur les plantes sont biologiquement moins actifs que ceux récoltés par les abeilles. Cependant, les stocks de pollens dans la ruche ne sont pas toujours considérables et les abeilles vivent parfois au jour le jour sans provisions importantes de pollen. Chauvis (I950) a montré que des remaniements journaliers ont lieu dans les cellules à pollen en période active de ponte et qu'il n'y a pas toujours de réserves importantes. Dans ces conditions, le pollen n'aurait pas toujours dans la colonie d'abeilles la même valeur alimentaire et biologique par suite de la durée très irrégulière du stockage. En ce qui me concerne j'ai montré que le facteur antibiotique royait son activité vavier au cours du temps. Voici un résumé de ces résultats :

Le pollen en pelote ou en rayon subit des transformations que l'on peut bloquer $\dot{a}-35^{\circ} \mathrm{C}$. 
Le pollen en rayons a déjà subi des transformations par rapport à celui des pelotes. Mais il peut subir encore des remaniements, puisqu'il se modifie même à $+\mathrm{I}^{\circ} \mathrm{C}$.

Le pollen en pelote conservé à température du laboratoire pendant plusieurs mois est plus actif que le même pollen conservé à $+\mathrm{I}^{\circ} \mathrm{C}$. Les transformations sont plus rapides dans la ruche à température $\left(34^{\circ} \mathrm{C}\right)$ et hygrométrie constantes et élevées.

D'autre part, le facteur antibiotique est stable pendant de très longues périodes, puisqu'il est possible de le retrouver I an après la récolte, dans le milieu si particulier de la ruche. Cette conservation est principalement intéressante pour l'élevage du couvain au début du printemps lorsque la récolte extérieure de pollen frais est encore insuffisante pour pallier aux besoins de la colonie. Cependant, il ne faut pas perdre de vue que le pollen n'est pas antifongique et qu'il est attaqué très souvent par des moisissures, dans les rayons inoccupés par les abeilles en période hivernale.

Je ne m'étendrai pas maintenant sur la comparaison de la valeur antibiotique du pollen, avec les autres produits de la ruche mais seulement dans le chapitre VII après avoir exposé les autres résultats concernant les antibiotiques de la colonie d'abeilles. Toutefois l'examen de la figure 27 nous permet de voir d'un seul coup d'œil les différences assez importantes qui existent entre le pollen et les matières que nous avons déjà étudiées, à savoir la propolis et la cire, principalement en ce qui concerne leur action antibiotique sur Salmonella gallinarum et Proteus vulgaris. Nous avons rencontré une activité antibiotique très variable suivant les différents pollens récoltés par les abeilles. Cette variabilité est due peut-être en partie à la valeur des pollens eux-mêmes, mais je pense que l'addition de substances par l'abeille, en quantités diverses suivant les pollens, renforce encore cette disparité. Certains pollens (Taraxacum) sont riches en lipides (I4,4 p. Ioo) et il faut peut-être moins de nectar pour les agglutiner sur les poils del'insecte. Ces faits expliqueraient en partie que les abeilles ajoutent plus ou moins de substances diverses lors de la récolte de tel ou tel pollen et que, par là-même, ceux-ci seraient assez différents du point de vue antibiotique. Quoi qu'il en soit le pollen est profondément modifié par rapport à son état initial dans la nature.

Je terminerai en signalant que le pollen est d'une grande importance dans la vie de l'abeille. Nous avons vu que la ruche en consomme 25 kilogrammes par an en moyenne. Je puis donc admettre que la quantité d'antibiotique ayant pour origine le pollen est considérable et que le pollen joue un rôle de premier plan dans l'hygiène de la ruche et dans la lutte contre les microbes pathogènes.

Enfin, n'y a-t-il pas lieu de mettre en évidence à nouveau l'équilibre qui règne dans la colonie d'abeilles lors de la récolte du pollen. L'échantillon moyen de pollen contient toujours le facteur antibactérien dans des 
proportions semblables, alors que triés isolément certains pollens sont très antibiotiques tandis que d'autres sont totalement inactifs. Les abeilles récoltent les uns et les autres en faisant un choix comme l'a vu Louveaux et ce choix n'est pas obligatoirement axé seulement sur la valeur nutritive, le facteur de croissance, le facteur réglant le développement des glandes ou le principe antibiotique; la récolte équilibrée faite par l'insecte répond plutôt à un ensemble de besoins, variable suivant le développement du groupe et la saison. 


\section{L'ANTIBIOTIQUE DU MIEL I'APIS IELLIFICA L.}

\section{A. - Généralités.}

Le miel entreposé dans les ruches provient d'une transformation et d'une concentration du nectar récolté sur les fleurs. Cette substance hydrocarbonée est l'aliment énergétique de l'abeille et lui permet notamment de produire de la chaleur et d'hiberner dans de bonnes conditions par les froids les plus rigoureux. La composition moyenne d'un miel peut se résumer par les chiffres suivants :

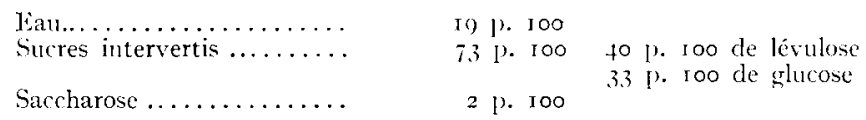

Le reste étant formé de dextrines, protéines, cendres, acides et environ $3,5 \mathrm{p}$. Ioo de constituants indéterminés. I e $\mathrm{pH}$ varie entre 3,6 et 4,2 .

Il est évident que la composition d'un miel varie suivant son origine florale ou géographique, sa teneur en eau et son temps de conservation. En effet, nous savons par exemple que plus un miel est vieux et moins il contient de saccharose (moitié moins 5 ans après la récolte). Cette transformation est due à l'influence de l'invertine; mais il en existe évidemment bien d'autres. Ia teneur en eau varie plus que les autres constituants. Les constituants non déterminés sont très importants du point de vue physiologique et font actuellement l'objet d'études très poussées.

Les hommes ont depuis toujours utilisé le miel non seulement comme nourriture, mais aussi pour ses propriétés antiseptiques, comme médicament, comme substance servant à la conservation des fruits et des graines, et jadis dans les embaumements (Egypte, Ancien Empire). L'utilisation du miel dans les embaumements humains repose sur l'observation de son action antiputréfiante. Ires notions empiriques de la valeur antiseptique du miel ont été confirmées scientifiquement ces dernières années. On a d'ailleurs essayé de donner de nombreuses explications à ce phénomène. On a invoqué la présence d'acide formique, mais on sait maintenant que celui-ci n'existe qu'à l'état de traces. D'après LochHEAD (I93I), c'est l'acidité du miel et la présence d'une quantité importante de sucres qui conféreraient au miel sa valeur antiseptique. STURTEVANT prétendait, de son côté, que c'est l'affinité du miel pour l'eau qui tue les microorganismes en les déshydratant. BUCHHEISTER pense qu'il s'agit 
d'une action leucotrope, tandis que Gonzenbach et Hofmana expliquent 1a guérison des plaies par une action physico-biologique du miel amenant un afflux de lymphe de 1'intérieur des tissus. Zaiss (I934) le premier a attiré l'attention sur le fait que le miel est un produit naturel comme le lait et qu'il peut posséder comme lui une action bactéricide propre. D'autre part, WHITE (IgO6) avait déjà montré que le miel provenant d'une ruche saine est toujours stérile et qu'on n'y rencontre jamais de bactéries sous forme végétative. D'après GABBERT (I936), on ne trouve dans le miel que des spores de bactéries en raison de l'inhibition des formes végétatives. C'est aux environs des années I930 que l'étude du facteur antibiotique du miel prend véritablement son essor. Dans cette littérature très abondante et où bien souvent les auteurs travaillent sur les mêmes sujets, on peut dégager quelques travaux de base que je vais étudier en détail.

GUNDEL et BlatTNer (I936) démontrent l'action cicatrisante du miel sur les plaies (très anciennement connue comme nous l'avons vu) et dissocient cette activité d'une action possible de ses diastases (catalase et oxydase).

Le miel a été étudié par I'RANCo et SARTORI (r940) et Franco (r94I). Ces auteurs ont établi son activité antibactériostatique et bactéricide sur divers microbes. Ils ont proposé comme explication soit l'action de la teneur en sucre, soit la présence du pollen transformé, soit le rôle du $\mathrm{pH}$, bien que ces divers éléments séparés ou combinés par l'expérimentateur soient moins actifs que le miel naturel. Ils ne pensent pas que la substance bactériostatique puisse provenir de l'abeille elle-même.

L'on peut admettre que les recherches les plus importantes sur le sujet ont été réalisées en Allemagne. Les travaux les plus marquants sont ceux de DoLD aux environs de I930, travaux poursuivis en collaboration avec divers auteurs pendant une dizaine d'années. DoLD (I932) montre que le miel renferme de l'inhibine, subtance sécrétée principalement par 1'organisme humain et présente dans la salive, le lait, les sécrétions bronchiques et nasales. DoL,D, Du et Dziao (1938) ont trouvé que l'inhibine $d u$ miel est responsable de l'activité antibactérienne et que cette activité n'est pas due aux acides, ni à la concentration en sucres, puisque des mélanges de ces substances (miel artificiel) n'ont pas la même action. De même, les diastases, inulase, invertase et catalase, ne possèdent pas d'activité antibiotique. Le $\mathrm{pH}$ du miel a été également mis hors de cause. Les auteurs n'ont pas trouvé de lysozyme dans le miel. L'inhibine serait thermolabile et photolabile.

Elle est détruite partiellement :

- en 2 minutes à $100^{\circ} \mathrm{C}$;

- en 5 minutes à $80^{\circ} \mathrm{C}$;

- en Io minutes à $70^{\circ} \mathrm{C}$;

- en 30 minutes à $5^{\circ} \mathrm{C}$; 
et inactivée après exposition en couches minces pendant I heure à la lumière solaire.

En revanche le facteur antibiotique se conserve longtemps dans le miel à l'obscurité et à température ordinaire. Il faut noter que sa destruction est rapide en dilution à $50 \mathrm{p}$. Ioo avec du sérum physiologique. L'inhibine s'extrait par l'acétone. Elle est aussi retenue par filtration poussée et adsorbée sur kaolin. Ėnfin un grand nombre de bactéries sont arrêtées dans leur croissance et même partiellement détruites par l'inhibine qui est inactive sur les champignons et les levures. DoLD et KNAPP (I949) ont effectué des recherches plus poussées sur la valeur antibactérienne du miel sur Corynebacterium diphteriae, agent pathogène responsable de la diphtérie. Il existerait deux facteurs dans le miel 1'un thermolabile, qui est inhibiteur du bacille (inhibine) et 1'autre qui serait thermostable et photostable qui provoquerait seulement une modification morphologique. Le miel artificiel est sans action inhibitrice sur le bacille d'Eberth, mais il possède les propriétés modificatrices du miel naturel. En outre, des essais cliniques in vivo ont confirmé que le miel appliqué en badigeonnage sur les amygdales ou instillé en solution dans le nez provoque une destruction des bacilles. En I955, DoLD et Witzenhausen codifient et publient la méthode de dosage de l'inhibine dans le miel que l'on mesure grâce au développement du Staphylococcus pyogenes aureus. Récemment DUISBERG et WARNECKE (I958) ont dosé l'inhibine dans 600 miels avec cette dernière méthode. Les résultats obtenus montrent qu'il n'y a aucun rapport entre la présence de l'inhibine et la couleur ou la consistance des miels. Au contraire, il y a un rapport assez net entre teneur en saccharase et inhibine, les miels à faible teneur en saccharase ayant une faible teneur en inhibine. A l'inverse, ils n'ont pas trouvé de rapport avec la teneur en amylase. Cependant, dans ce travail, on n'a pas étudié l'action de la chaleur sur l'inhibine.

D'un autre côté, SAckETT aux Ftats-Unis montre que le miel est actif sur les bacilles de la fièvre typhoïde, de la dysenterie et des diarrhées variées. Il trouve aussi que Proteus vulgaris est détruit en 4 jours et le Bacillus typhosus responsable de la fièvre typhoïde en 48 heures.

En France, LEmay (I947) étudie quelques échantillons de miel du point de vue bactériologique. Il y trouve quelques germes banaux du type Subtilis, pas d'Entérobactériacées, rarement des diplocoques, non pathogènes. De plus il établit l'action bactériostatique et même bactéricide du miel envers le colibacille et les staphylocoques. En I95 I, VERGÉ étudie à la Station de Recherches Apicoles de Bures et à 1'Institut Pasteur 1'action de l'inhibine du miel. Il retrouve les résultats des auteurs déjà cités et obtient des résultats positifs avec des solutions 
aqueuses de miel, mais aussi avec des extraits alcooliques, éthérés et acétoniques. Il résulte de ces expériences que les Salmonella sont moins sensibles que les autres souches essayées, d'autre part que les extraits acétoniques ont une action inhibitrice prédominante et enfin que les divers extraits subissent une décroissance d'activité en fonction de l'exposition à la lumière et de la chaleur. I'auteur pense également que le passage à 1'autoclavage du miel dilué est responsable de la destruction de la substance antibiotique et thermolabile.

En 1956, HELLEU qui étudie 1'action du miel sur Escherichia coli, Eberthella typhosa et Staphylococcus aureus trouve que le miel n'est pas actif sur ces souches; nous verrons plus loin que $E$. coli était d'ailleurs mal choisi pour ce travail. Dans le même ordre d'idées, IAANGLADE, Hinglais (H.) et Hinglais (M.) en I957 n'ont pas trouvé dans le miel un germicide actif sur le Bacille de Koch.

Nous savons d'autre part depuis peu (MAurizio, I958) que le miel naturel même bouilli est un puissant inhibiteur de la germination du pollen. Cette inhibition n'est pas due uniquement à l'acidité ni à la présence des sucres, elle est complétée par un autre facteur. Faut-il rapprocher cette action de l'activité antigerminative du miel sur les graines, bien connue depuis longtemps? J'analyserai plus loin tous ces résultats en les comparant avec mes essais personnels. Le miel sert également dans la conservation des fruits et déjà Columelle en conseillait l'emploi dans son ouvrage "De Arboribus ". Faut-il voir dans cette dernière utilisation, encore prônée dans certaines provinces, une action antibactérienne ou antigerminative du miel? La question n'est pas résolue à 1'heure actuelle.

\section{H. - Travaux personnels.}

I Matériel et méthodes.

J'ai repris l'étude de la valeur antibactérienne des miels mais avec une méthode bien différente de celle de DoLD et WITZENHAUSEN (I955). Voici tout d'abord un résumé de la technique de ces derniers.

On prépare un milieu à la gélose à 3 p. Ioo (milieu hématine-agar) que l'on distribue stérilement en tubes. Les échantillons de miel sont dilués à 50 p. Ioo avec du sérum physiologique stérile. Le miel est ensuite ajouté au milieu chauffé : le tout, mis en boîte de Pétri de manière à obtenir, pour chaquelot I5 $\mathrm{ml}$ de milieu au total, les concentrations en miel dans le milieu étant de 25 p. IOO, 20 p. IOO, I5 p. IOO, IO p. IOO et $5 \mathrm{p}$. roo. On laisse refroidir avant d'ensemencer. Le Staphylococcus pyogenes aureus a été choisi pour l'épreuve. On prépare une suspension 
bactérienne diluée : on ensemence en surface le milieu le mieux possible et l'on porte à $37^{\circ}$. La lecture des résultats à lieu 24 heures après. On note de o à 5 pour la valeur inhibitrice d'après le tableau suivant :

Inhibition totale pour $5 \mathrm{p}$. Ioo de miel $=$ note 5 .

Inhibition totale pour ro p. roo de miel $=$ note 4 .

Inhibition totale pour $I_{5}$ p. Ioo de miel $=$ note 3 .

Inhibition totale pour $20 \mathrm{p}$. Ioo de miel $=$ note 2 .

Inhibition totale pour $25 \mathrm{p}$. Ioo de miel $=$ note $\mathrm{I}$.

Pas d'inhibition

$=$ note 0 .

Les miels sur lesquels j'ai travaillé moi-même sont des miels bien identifiés et le plus souvent récoltés par les ruches du laboratoire, conservés à température ordinaire et à l'obscurité. Je n'appellerai pas inhibine le facteur antibiotique que j'ai trouvé dans les miels. En effet, d'après tous les auteurs qui m'ont précédé, l'inhibine est considérée comme thermolabile et photolabile. De mon côté, de nombreux essais répétés m'ont montré que le principe antibiotique que j'ai mis en évidence est photolabile mais thermostable. Il s'agit peut-être d'un même facteur, modifié par les méthodes d'extraction et d'épreuves différentes, mais rien ne le prouve à l'heure actuelle. L'antibiotique du miel s'extrait par l'eau, mais il s'extrait d'une manière plus facile par l'acétone à froid. C'est la méthode que nous avons employée dans la majorité de nos essais. L'extrait éthéré est complètement inactif ; d'ailleurs KITZES, ScHUETTE et ELVEHJEM (I943) ont déjà signalé l'absence d'activité des extraits éthérés de miel (aussi bien du point de vue bactériostatique que bactériostimulant). Mes extraits acétoniques ont porté sur du miel pur et non dilué dans le sérum physiologique comme dans les essais de Doud. Le miel est trituré à froid au mortier (après légère macération) par 3 fois son volume d'acétone, de façon à bien extraire la totalité de la substance active. Après un tel traitement, il est impossible en renouvelant l'opération d'obtenir un supplément de substance active. L'extrait acétonique, presque incolore, est évaporé, au bain-marie et le résidu repris par l'eau. Ën solution aqueuse, l'extrait possède une odeur de miel très forte et qui dure de longs mois. Cette liqueur est alors ajoutée en quantité croissante à des milieux de culture gélosés pour être éprouvée par la méthode de la dilution. I,es unités antibiotiques sont calculées de la même façon que précédemment en milieu aqueux; cette substance est très stable au réfrigérateur (à $0^{\circ}$ ) et à l'obscurité. Un extrait de miel de colza a vu ses Unités subtilis passer de II,9 à 9,7 après deux ans de conservation, c'est-àdire que la perte d'activité antibiotique est négligeable. Au contraire, les extraits de miel sont photolabiles et détruits par l'exposition à la lumière solaire en peu de temps. 


\section{$2^{\circ}$ Résultats obtenus.}

J'ai tout d'abord essayé les extraits de miel d'origines diverses sur Bacillus subtilis souche Caron. Ces miels nous ont montré en général une similitude d'actions antibiotiques. Il y a très peu de différence en général d'un miel à l'autre, cependant il est possible d'enregistrer parfois des variations assez importantes sans que je puisse en fournir ici l'explication. La figure 3I montre 1'activité antibiotique de quelques miels sur Bacillus subtilis. On y remarque qu'un miel de lavandin est pauvre en substance inhibitrice, alors qu'un miel mélangé de la même année et de la même région est au contraire beaucoup plus efficace.

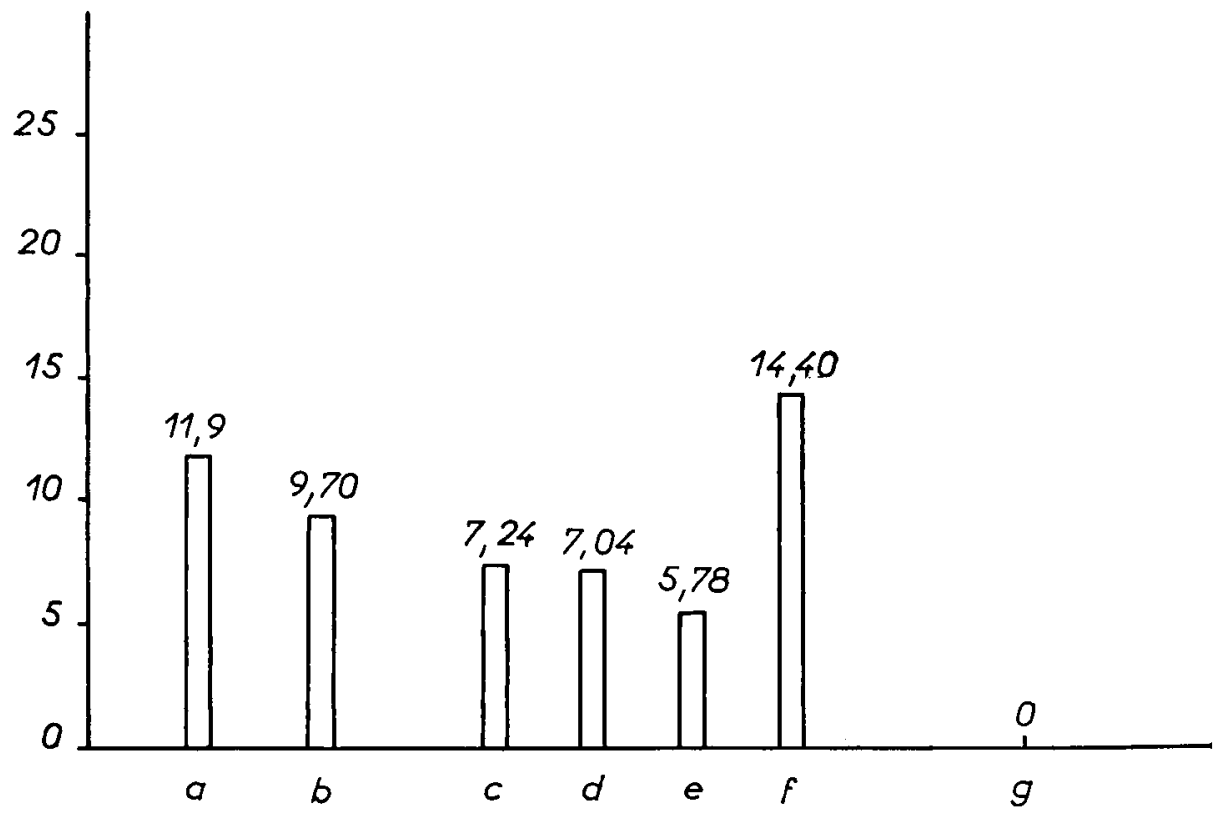

FIG. 31. - Valeur antibiotique de divers miels (épreuve B. subitis).

$$
\begin{aligned}
& a: \text { miel de colza frais. } \\
& b \text { : miel de colza (extrait de } 2 \text { ans.) } \\
& c: \text { miel de la Crau. } \\
& d: \text { miellat de sapin (Jura). } \\
& c: \text { miel de lavandin. } \\
& f: \text { miels divers én mélange. } \\
& g: \text { extraits éthérés de miels et miellats. }
\end{aligned}
$$

Dans la figure 27, est notée l'action du principe antibiotique du miel par rapport à ceux du pollen, de la cire et de la propolis. Les unités antibiotiques sont calculées comme je l'ai déjà expliqué en prenant comme base la valeur $\mathrm{U}=$ Io pour l'action obtenue sur Subtilis de façon à pouvoir comparer les extraits entre eux. Il est facile de se rendre compte que l'action du principe antibiotique du miel est principalement marquée sur : Bacillus alvei, Proteus vulgaris et Bacillus subtilis. Cette action est 
moitié moindre sur les Salmonella, les Escherichia coli Bordet et Escherichia coli B et le Bacillus larvae. L'activité inhibitrice sur Psendomonas pyocyanea est très faible, mais si l'on opère en dessous de la dilution limite nécessaire à cette inhibition, on met en évidence une action retardatrice $d u$ développement de la souche. J'ai éprouvé aussi l'extrait acétonique de miel sur une dizaine de souches d'Escherichia coli, dont trois seulement sont notées sur la figure 27. I'action sur Escherichia coli est nulle, quelle que soit la concentration employée (souches no Io à Ig inclus du tableau I). A l'inverse de ce que j'ai remarqué pour les autres bactéries, j'ai constaté d'une manière habituelle que l'extrait ajouté à faibles doses favorisait le développement des Escherichia coli par rapport aux témoins. Il est possible qu'il existe dans l'extrait acétonique du miel un facteur de croissance actif sur Escherichia coli qui neutralise l'action de l'antibiotique. Schuler et Voger (I956) ont montré de leur côté que l'inhibine favorisait le développement des bactéries de la gastro-entérite. Au contraire, j'ai trouvé que cette substance de croissance est thermostable et non thermolabile comme le soutiennent ces deux derniers auteurs qui travaillent vraisemblablement avec la méthode de Dor,D.

Afin de contrôler l'influence de la chaleur sur le facteur antibiotique du miel, j'ai essayé des extraits acétoniques de miels chauffés au préalable à des températures et pendant des temps définis, sur deux souches bactériennes. Les résultats sont assez difficiles à interpréter, je les ai portés sur le tableau XVI. Cependant, malgré quelques chiffres aberrants, il semble se dégager les faits principaux suivants :

ro Tous les miels chaufés sans exception fournissent des extraits contenant un facteur antibiotique.

$2^{\circ}$ Ce facteur inhibiteur ne faiblit pas si l'on augmente la température (entre la température ordinaire du laboratoire et $100^{\circ} \mathrm{C}$ ), même après chauffage de 30 minutes.

$3^{0}$ Mais si 1'on chauffe I heure aux mêmes températures que ci-dessus, 1'action inhibitrice sur Bacillus subtilis baisse assez sérieusement, tandis qu'elle augmente vis-à-vis de Proteus vulgaris.

$4^{\circ}$ D'autre part, il existe un rapport direct entre le poids de l'extrait sec obtenu après extraction et le temps de chauffage du miel. Tous les extraits de miels ayant été chauffés 30 minutes, sont plus riches en poids sec par centimètre cube que ceux chauffés I heure et ceux-ci plus que ceux chauffés $12 / 24$ ou 36 heures.

$5^{\circ}$ Le chauffage du miel à $120^{\circ}$ pendant I $_{5}$ minutes à 1'autoclave ne détruit pas le pouvoir inhibiteur des extraits acétoniques.

$6^{\circ}$ Le chauffage prolongé à $80^{\circ}$ pendant 12,24 ou 36 heures montre une action accrue sur Proteus vulgaris. L'action sur Bacillus subtilis étant moitié moindre.

Ces résultats sont très différents de ceux qui ont été obtenus par mes 
prédécesseurs, mais il ne faut pas oublier que j'ai chauffé des miels et non des solutions de miel dans le sérum physiologique. I,a différence de méthode est importante, car le miel dilué à 50 p. Ioo perd son activité assez rapidement même à température ordinaire.

TABLEAU XVI

Influence du chauffage sur le miel.

\begin{tabular}{|c|c|c|c|c|}
\hline \multirow{2}{*}{ Temps de chauffage } & \multirow{2}{*}{$\begin{array}{l}\text { Température de } \\
\text { chauffage en de- } \\
\text { grés centigrades }\end{array}$} & \multirow{2}{*}{$\begin{array}{l}\text { Poids sec en mg } \\
\text { dans I cl d'extrait }\end{array}$} & \multicolumn{2}{|c|}{$\begin{array}{c}\text { Action des extraits obtenus en Unités } \\
\text { antibiotiques }\end{array}$} \\
\hline & & & sur l3acillus subtilis & sur Proteus vulgaris \\
\hline \multirow[t]{3}{*}{ Témoin non chauffé } & & $8 r$ & 6,2 & 6,2 \\
\hline & $50^{\circ}$ & 82 & 6,0 & $\mathrm{I} 2, \mathrm{I}$ \\
\hline & $64^{\circ}$ & 98 & I 0,2 & 10,2 \\
\hline \multirow[t]{3}{*}{30 minutes } & $75^{\circ}$ & 97 & ro, 3 & 10,3 \\
\hline & $90^{\circ}$ & 94 & Io,5 & 10,5 \\
\hline & $98^{\circ}$ & 92 & 10,8 & 10,8 \\
\hline \multirow{5}{*}{ I heure } & $5^{\circ}$ & 82 & 5,2 & I0,5 \\
\hline & $64^{\circ}$ & 75 & 6,6 & I 3,3 \\
\hline & $75^{\circ}$ & 74 & 6.7 & $\mathrm{I} 3,5$ \\
\hline & $90^{\circ}$ & 58 & 8,6 & I 7,2 \\
\hline & $9^{8^{\circ}}$ & 68 & 7,3 & I 4,5 \\
\hline I 2 heures & $80^{\circ}$ & 45 & 9,8 & 19,6 \\
\hline $3^{6}$ heures & $80^{\circ}$ & 50 & 9,9 & I 9,8 \\
\hline
\end{tabular}

J'ai pratiqué l'épreuve suivant les méthodes de DoL,D, sur un miel récolté par nos soins. Ce miel est dilué dans du sérum physiologique et on prépare des milieux avec addition de gélose de manière à ramener les teneurs en miel à : 5 p. IOO, Io p. IOO, I5 p. I00, 20 p. I00, 25 p. IOO de miel. L.es mêmes dilutions ont été réalisées après un chauffage à $80^{\circ}$ pendant 30 minutes. Les résultats obtenus sur Bacillus subtilis sont les suivants :

Io Le miel non chauffé nous a donné (suivant la notation de DoLD et de Witzenhausen) une valeur inhibitrice de 3,5.

$2^{\circ}$ Le même miel chauffé nous a donné une valeur inhibitrice de 2 .

$3^{\circ}$ Un milieu témoin contenant $25 \mathrm{p}$. Ioo de saccharose permet le développement normal de Bacillus subtilis.

J'ai donc montré que s'il y avait une baisse de l'activité de la substance antibiotique $d u$ miel par le chauffage, il n'y a pas une destruction totale même pour un miel chauffé à $80^{\circ}$ et pendant une demi-heure. 
$3^{\circ}$ Comparaison entre mes résultats et ceux des autres auteurs.

Il est toujours très difficile de rapprocher des résultats obtenus par différentes méthodes, cependant un certain nombre de souches bactériennes éprouvées sont les mêmes, ce qui facilite la comparaison.

Tout d'abord, nous avons déjà vu la similitude des résultats de Schuler et VOGEL et des miens en ce qui concerne le facteur de croissance présent dans le miel qui accélère et favorise la croissance de certaines Entérobactériacées.

J'ai trouvé également comme VERGÉ (I95I), KITZES, ScHUETTE et EIVEHJEM que les extraits éthérés de miel sont inactifs.

\section{TABLEAU XVII}

Action antibactérienne des facteurs présents dans le miel d'après quelques auteurs.

Miel naturel non chauffé.

\begin{tabular}{|c|c|c|c|c|c|c|c|}
\hline \multirow{3}{*}{$\begin{array}{c}\text { Souches bactériennes } \\
\text { essayées }\end{array}$} & \multirow{3}{*}{ Franco } & \multicolumn{3}{|c|}{ Dold, Du et Dziao } & \multirow{3}{*}{ Vergé } & \multirow{3}{*}{ Schuler et Vogel } & \multirow{3}{*}{ Lavie } \\
\hline & & \multicolumn{3}{|c|}{$\begin{array}{c}\text { Concentration en miel } \\
\text { dans le milieu }\end{array}$} & & & \\
\hline & & I $7 \%$ & I $3 \%$ & 10 $\%$ & & & \\
\hline Bacillus subtilis....... & & & + & +- & + & & + \\
\hline \multicolumn{2}{|l|}{ Bacillus alvei ......... } & & \multirow[b]{2}{*}{$-\overline{+}$} & \multirow{2}{*}{+-} & + & & + \\
\hline Pseudomonas pyocyanea & \multirow{2}{*}{$\begin{array}{l}+ \\
+ \\
+\end{array}$} & 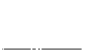 & & & \multirow{2}{*}{$\begin{array}{l}+ \\
+\cdots \\
+\cdots\end{array}$} & 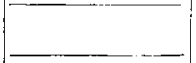 & +- \\
\hline Salmonellae .......... & & \multirow{2}{*}{+-} & + & +- & & & +- \\
\hline Entérobactériacées ..... & + & & $\longrightarrow$ & - & + & - & - \\
\hline \multirow{2}{*}{ Observations } & \multirow{2}{*}{ 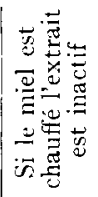 } & \multirow{2}{*}{\multicolumn{3}{|c|}{$\begin{array}{c}30 \text { minutes à } 5^{\circ} \\
\text { suffisent à détruire } \\
\text { le facteur antibiotique }\end{array}$}} & \multirow{2}{*}{ 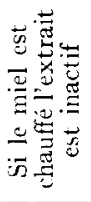 } & \multicolumn{2}{|c|}{$\begin{array}{l}\text { L'effet sur les entérobactériacées } \\
\text { est opposé }: \text { il existe un facteur } \\
\text { de croissance }\end{array}$} \\
\hline & & & & & & $\begin{array}{l}\text { Si le miel est } \\
\text { chauffé l'extrait } \\
\text { est inactif }\end{array}$ & $\begin{array}{l}\text { Si le miel est } \\
\text { chauffé l'extrait } \\
\text { reste actif }\end{array}$ \\
\hline
\end{tabular}

SACKE'T'T avait montré comme moi-même l'action bactériostatique et même bactéricide des extraits de miels sur Proteus vulgaris. I,e tableau XVII permet de confronter quelques résultats. Nous retrouvons une action nette sur Bacillus subtilis et Bacillus alvei, une activité plus faible sur les Salmonellae et le Pseudomonas pyocyanea. En ce qui concerne les Entérobactériacées, je retrouve comme SchulER et VogEL, un facteur qui favorise la croissance pour ces bactéries. DoLD trouve sur les mêmes très peu d'activité inhibitrice sauf s'il ajoute I7 p. roo de miel à son milieu. S’il ajoute seulement 13 p. Ioo, ou ro p. Ioo de miel il 
n'obtient aucune inhibition. VERGÉ et F'RANCO ont trouvé une action antibiotique sur Escherichia coli, mais je ne possède pas le protocole de leurs expériences. Au contraire, pour l'ensemble de mes résultats, je ne suis d'accord avec aucun des auteurs précités sur la destruction du principe antibiotique par le chauffage du miel. En effet DOLD, DU et DzIAO admettent qu'un chauffage à $56^{\circ}$ pendant 30 minutes, à $70^{\circ}$ pendant ro minutes ou encore à $80^{\circ}$ pendant 5 minutes, suffit à détruire le facteur antibactérien du miel. Les travaux récents de Schade, Marsh et Ëcker'T (1956) ont montré que l'inhibine était plus sensible que les diastases à la chaleur et qu'elle était totalement détruite au bout de I $_{5}$ jours de conservation à $55^{\circ}-60^{\circ}$. Cependant, les auteurs ne sont pas d'accord avec les travaux allemands sur le principe que le dosage de l'inhibine puisse servir à reconnaître un miel chauffé.

\section{$4^{\circ}$ Origine du facteur antibiotique du miel.}

Cette question n'est pas encore résolue totalement, cependant comme nous l'avons vu (et je suis loin de les avoir tous cités) les travaux sur le miel du point de vue antibiotique sont fort nombreux. DoLd (I938) a recherché si l'origine de l'inhibine du miel était végétale ou au contraire si elle provenait de l'abeille elle-même. Les quelques essais faits à cette époque notamment sur certains végétaux n'ont pas éclairé le problème; l'auteur a trouvé de l'inhibine chez la pomme de terre et il a même établi que les jeunes tubercules étaient plus riches que les vieux.

On peut supposer que le facteur inhibiteur du miel provient soit des sécrétions salivaires de 1'abeille, soit de la présence au contact du miel de la cire, de la propolis ou du pollen. L'examen de la figure 27 , par exemple, ne permet pas pourtant de trouver des relations directes entre le miel et les autres produits de la ruche. Les extraits de miel sont très différents des extraits de pollens du point de vue antibiotique; d'autre part le pollen est présent dans le miel en quantité si faible qu'il ne pourrait modifier sa valeur antibactérienne. La comparaison des extraits de propolis avec ceux du miel ne donne pas non plus d'explications satisfaisantes. Les extraits qui se ressemblent le plus du point de vue antibiotique sont certainement ceux de la cire et ceux du miel. Principalement, les liqueurs extraites de la cire par l'eau bouillante (voir tableau $\mathrm{X}$ ). I es courbes de la figure 27 sont presque parallèles. Ia seule différence notable est que les extraits de cire sont inactifs sur Pseudomonas pyocyanea. On pourrait aisément concevoir une action de la cire sur le miel puisqu'il est entreposé dans les cellules. De plus, la taille des cellules de cire fait que le miel d'un rayon est en contact avec une très grande surface de cire et il se pourrait qu'il y ait une diffusion de la substance (hydrosoluble) dans le miel. D'autre part, si l'on compare l'action des extraits de niel et celle des 
extraits de l'abeille, on y trouve de grosses différences en ce qui concerne l'inhibition des Escherichia coli, l'extrait d'abeille étant actif sur les Io souches éprouvées à l'inverse de l'extrait de miel qui ne l'est jamais. D'autre part, l'extrait d'abeille est deux fois plus actif sur Salmonella pullorum et Salmonella gallinarum que sur Bacillus subtilis, tandis que les extraits de miel sont deux fois moins actifs sur ces deux Salmonella que sur Bacillus subtilis. Il me semble donc que les deux substances sont différentes.

J'ai essayé d'éclaircir le problème de l'origine de l'antibiotique du miel de deux manières.

Tout d'abord, j'ai éprouvé la valeur antibiotique du nectar sur Bacillus subtilis. I a difficulté principale de ces essais vient de ce qu'il est en général difficile de récolter une grande quantité de nectar sur des fleurs correspondant à un échantillon de miel de la même origine. L'addition de 30 p. Ioo à 50 p. Ioo d'un nectar de lavandin (contenant 40 p. I00 de matière sèche) à un milieu de culture n'a pas permis de déceler une action antibiotique. Au contraire, le nectar prélevé sur Salvia sclarea I. (contenant 40 p. Ioo de matière sèche) inhibe le développement de Bacillus subtilis si la concentration est de $45 \mathrm{p}$. Ioo dans le milieu de culture. Cette action, en tenant compte de la teneur en eau plus élevée, que pour un miel, permet suivant la méthode de DoL, de noter l'activité de ce nectar à $I, 5$ ce qui est malgré tout assez faible vis-à-vis d'un miel normal. I'activité antibiotique d'un nectar de lavandin, venant d'être entreposé dans les cellules et contenant encore 30,5 p. Ioo d'eau, se situe dans l'échelle de Dold avec la note 3,5 bien que n'ayant pas encore atteint la concentration normale en eau d'un miel. J'étais obligé de conclure dès ces premiers essais que l'abeille ajoutait certainement une substance antibiotique au nectar et qu'un simple passage dans le jabot augmentait dans de grandes proportions la valeur antibiotique de la matière sucrée récoltée sur les fleurs.

C'est alors que j'ai réalisé les expériences ci-dessous qui démontrent d'une façon catégorique que l'abeille ajoute au nectar des substances antibiotiques, ou bien qu'elle ajoute des substances qui transforment ce nectar. Du sirop de sucre (6r,5 p. Ioo de matière sèche) est éprouvé par la méthode de DoL, sur Bacillus subtilis; il n'existe aucune action antibiotique, note o. Ce même sirop est ensuite donné en nourrissement à une colonie d'abeilles en période de disette ayant son corps de ruche déjà garni d'abeilles et dotée d'une hausse vide, sans aucune trace de miel. J'ai fait prendre très rapidement ce sirop aux abeilles et en grande quantité (3o litres). Dès que ce sirop est stocké en hausse, il est extrait, sans séjourner dans la ruche. I'épreuve de ce sirop ( $78 \mathrm{p}$. Ioo de matière sèche) qui a subi un passage dans le jabot de l'abeille montre qu'il est légèrement antibiotique sur Bacillus subtilis; note 0,5 . Il était possible également de nourrir à nouveau des abeilles avec le sirop actif une 
ou plusieurs fois et d'essayer chaque fois sur notre bactérie sa valeur antibiotique. Après un deuxième passage dans le jabot (8r p. Ioo de matière sèche) la note suivant DoLD est de 3 et après un troisième passage ( $82 \mathrm{p}$. Ioo de matière sèche) la note 3,5 est atteinte pour la valeur antibiotique de ce sirop. J'ai donc montré que l'abeille ajoute au sirop de sucre (et par conséquent au nectar) des substances antibiotiques et que chaque passage dans le jabot augmente l'activité antibactérienne de ce sirop. J'ai essayé en même temps la valeur des extraits acétoniques de ces différents sirops de sucre sur Bacillus subtilis et les résultats obtenus vont dans le même sens.

Cependant, la présence de substances antibiotiques dans certains nectars montre que l'origine de l'antibiotique des miels n'est pas toujours unique.

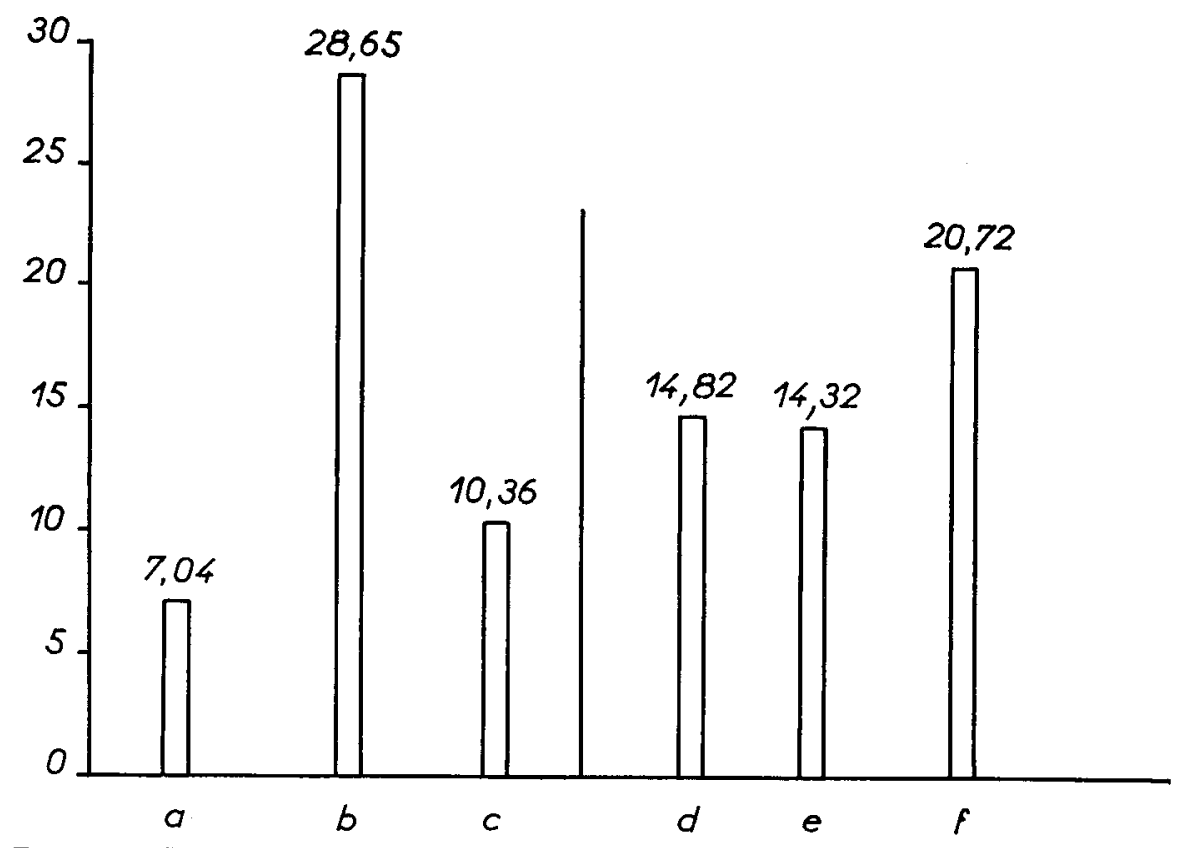

Fig. 32. - Comparaison de la valeur antibiotique de miellat de sapin et de différentes parties de l'arbre $(a, b$, et $c$ épreuves sur $B$. Subilits, $d$, e et $f$ épreuve sur Proteus vulgaris $)$.

$a$ et $d$ : extrait de miellat.

$b$ et $e$ : extrait de bourgeons.

$c$ et $f$ : extrait d'aiguilles.

\section{$5^{\circ}$ Valeur antibiotique du miellat.}

Le miellat est une déjection sucrée de pucerons qui est récoltée par l'abeille sur les aiguilles de sapins (Abies pectinata) dans certaines conditions de climat et d'altitude. Son origine étant très différente du miel, puisqu'il ne s'agit pas de nectar de fleur, il m'a semblé intéressant d'étudier cette substance du point de vue antibiotique. La figure 32 montre les résultats obtenus sur Bacillus subtilis et sur Proteus vulgaris avec des 
extraits acétoniques de miellat de sapin et de plus avec des extraits de bourgeons et des extraits d'aiguilles de sapin. I a substance extraite du miellat a une activité antibiotique comparable aux miels et notamment se rapproche de l'extrait de miel de Crau (voir figure 3I). I'extrait d'aiguilles d'A bies montre une action parallèle au miellat quoique légèrement plus forte que ce dernier. Les extraits des bourgeons de sapin ne réagissent pas du tout de la même manière et ces derniers sont principalement actifs sur Bacillus subtilis. Il est cependant difficile d'affirmer que le principe antibactérien présent dans le miellat a pour origine 1'aiguille de sapin. Il est vraisemblable que le puceron lui aussi modifie les substances lors du passage dans son tube digestif. En résumé, je puis dire seulement que le miellat semble se comporter comme les miels du point de vue de leur teneur en principes antibiotiques. D'autre part, les extraits éthérés de miellat, pas plus que ceux de miels, ne possèdent d'activité antibactérienne.

\section{$6^{\circ}$ Action antibiotique du miel sur les bactéries responsables des loques.}

L'action du facteur antibiotique du miel a été étudiée en rapport avec deux souches bactériennes pathogènes de la ruche; Bacillus alvei et Bacillus larvae. L'extrait de miel sur Bacillus alvei est trois fois plus actif que sur Bacillus larvae et une fois et demie plus actif que sur Bacillus subtilis. Ce facteur antibiotique est plus actif que l'extrait d'abeille ( 2 fois pour alvei et 3 fois pour larvae), mais beaucoup moins intéressant que les extraits de pollens et principalement de cire et de propolis. J'ai constaté sur Bacillus larvae un effet retardateur dans le développement avec l'extrait acétonique du miel lorsque l'on se trouve en dessus de la dilution limite qui amène l'inhibition totale. Ce retard est de 36 heures avec roo $\mathrm{mg}$ d'extrait sec dans Io cl de milieu et de $\mathrm{x} 2$ heures avec moins de Ioo $\mathrm{mg}$ d'extrait sec dans ro cl de milieur.

\section{$7^{\circ}$ Valeur antifongique du miel.}

I'action antifongique des extraits de miel a été éprouvée. Il n'existe pas de facteur antifongique dans les miels. J'ai pu remarquer que des moisissures diverses (Penicillium, Aspergillus, Mucor) se développaient très bien sur des milieux préparés avec l'extrait acétonique de miel pour l'épreuve de germination des graines. Mais, dans la ruche, les champignons ou les levures ne se développent pas sur le miel dans les conditions normales, sauf dans un miel trop riche en eau, peu concentré et qui de ce fait arrivera à fermenter. C'est la concentration en sucre du miel qui empêche en temps normal le développement fongique et non le facteur que l'on trouve dans la fraction antibiotique. Les travaux de DoLD (1938) ont d'ailleurs égale- 
ment confirmé ce point de vue. Des épreuves ont été pratiquées sur Penicillium, Aspergillus mucor, et la levure de bière au moyen de milieux synthétiques à base de miel. Il n'existe pas d'inhibition vis-à-vis du développement de ces champignons même si l'on ajoute jusqu'à I7 p. roo de miel dans le milieu de culture.

\section{$8^{\circ}$ Action antigerminative du miel sur les graines.}

Nous avons vu dans les généralités que le miel était utilisé jadis pour conserver les graines et les fruits : j'ai voulu contrôler l'action de l'extrait acétonique $\mathrm{d} u$ miel (repris en phase aqueuse) sur les graines. Je me suis servi de graines de riz disposées sur du coton hydrophile ou du papier filtre dans des boîtes de pétri par lots de roo. Un extrait acétonique de miel était évaporé et repris par l'eau dans les mêmes proportions que celles utilisées pour les essais antibiotiques. La liqueur servait ensuite à 1'humidification des graines en vue de leur germination ; les graines témoins étant arrosées à l'eau pure. I,e résultat est que l'extrait contenant le facteur antibactérien du miel contient également un facteur d'inhibition de la germination des grains de riz. La faible quantité d'extrait ajoutée à l'eau d'arrosage met hors de cause l'action du pH de la liqueur : d'autre part, il n'y a pas de sucres dans l'extrait. Cette inhibition de germination est due à un facteur qui est peut-être le même que 1'antibiotique. Je crois être le premier à avoir démontré scientifiquement (et à l'aide d'un extrait de miel) le bien-fondé de l'antique méthode de conservation des graines dans le miel. L'emploi de l'extrait démontre que les sucres ne peuvent être les seuls agents responsables.

\section{C. - Conclusion du chapitre IV.}

Il est établi à l'heure actuelle que le miel est une substance quasi dépourvue de microorganismes en activité, antibactérienne, antigerminative pour les grains de pollens et pour certaines graines. I,es travaux les plus récents ont balayé les vieilles hypothèses concernant les modes d'action antibactérienne du miel ( $\mathrm{pH}$, concentration en sucres, effet de déshydratation, etc.). On a démontré qu'il existe dans le miel un ou plusieurs facteurs antibiotiques qui, suivant le mode opératoire sont thermostables ou thermolabiles, mais toujours photolabiles. I,es mêmes extraits sont inhibiteurs sur le développement des bactéries et sur la germination des graines. L'extrait acétonique de miel n'a pas été essayé sur la germination du pollen, c'est le miel lui-même qui était ajouté au milieu (Maurizio).

Il résulte de cet ensemble de recherches que la valeur du miel est bien établie en ce qui concerne le pouvoir antibiotiqute. Je rappelle rapidement 
les utilisations médicales du miel qui sont innombrables et sans doute bien justifiées (voir revue de ChAuvis).

Les travaux de GUNDEL et BLAT'TNER ont montré l'efficacité du miel dans la guérison des plaies superficielles. I,es recherches de DoLD et KNAPP (I949) ont prouvé d'une manière certaine l'action bactéricide du miel sur l'agent pathogène de la diphtérie (Corynebacterium diphteriae). Enfin de nombreux auteurs et moi-même avons établi la valeur antibiotique du miel vis-à-vis de nombreuses bactéries pathogènes pour l'homme et les animaux domestiques. Clement et ICHEs (I9I2) citent les observations cliniques de DERNADr. Ce dernier aurait provoqué grâce à l'absorption de miel, l'arrêt de diarrhées rebelles et la reprise du poids dans des cas de tuberculoses pulmonaires. Les mêmes auteurs signalent l'action du miel sur les gastro-entérites humaines, sur le bacille diphtérique, sur les aphtes et sur la diarrhée des volailles. Des exemples innombrables ont depuis corroboré ces faits. SPÖTTEL (I950) dans un ouvrage assez complet dresse la liste des actions bénéfiques du miel. Il cite des cas de guérison de malades porteurs de germes de bacilles diphtériques, des cas de guérison de blessures et d'abcès ouverts. Mais, d'autre part, le miel stimule la croissance de la flore bactérienne de l'intestin. Donc, l'effet bactéricide du miel provient d'un autre facteur. L'origine du miel serait très importante et gouvernerait son activité spécifique. Je n'ai pas trouvé de mon côté de grandes différences entre divers miels du point de vue antibiotique, mais Dol, D, Du et DzIAo ont prétendu que l'inhibition n'était pas constante dans les miels et même qu'elle pouvait manquer totalement. Ces résultats ont été retrouvés par WARNEKE et DuisBERG (I958) lors de l'analyse de nombreux échantillons de miels.

\section{Importance biologique pour l'abeille.}

Dans la colonie d'abeilles, l'action antibactérienne du miel ne semble intéressante que pour sa propre conservation. En effet, le facteur antibiotique du miel est bien actif sur Bacillus larvae et surtout Bacillus alvei, mais beaucoup moins que les antibiotiques présents dans les autres produits de la ruche. D'autre part, la nourriture larvaire est soit de la gelée royale, soit une bouillie larvaire où le miel n'est pas le seul constituant; et son rôle antibiotique éventuel lors de la nutrition des larves est dépassé par les facteurs similaires de la gelée royale, des abeilles adultes et principalement des pollens stockés dans la ruche. Il faut noter également que le miel contient peu d'unités antibiotiques actives par rapport au poids initial de matériel extrait. Cependant, à l'intérieur de la ruche, il est possible que l'activité antibiotique du miel, renforcée par son $\mathrm{pH}$ et sa haute teneur en sucres soit d'un intérêt non négligeable. 
ChapiTRE $\mathrm{V}$

\title{
L'ANTIBIOTIQUE DE LA GELÉE ROYALE
}

\author{
A.' - Généralités. \\ I Origine, signification, composition.
}

Comme la cire, la gelée royale est une sécrétion del'abeille. De ce fait, cette substance pourrait sembler dès l'abord très différente des produits de la ruche récoltés sur les végétaux. Cependant, la gelée royale est en rapport direct avec la consommation de pollen, matière première protéique de la ruche. Nous avons vu dans la première partie que les nourrices possédaient des glandes pharyngiennes très développées, capables d'une production importante de gelée royale. Cette substance sert à la nourriture des très jeunes lavves. Après trois jours les larves d'ouvrières sont nourries avec "le pain d'abeilles", mélange mal défini de miel, pollen, sécrétions salivaires, de composition variable, et seules les larves royales destinées à l'obtention des reines continuent à recevoir uniquement la substance primitive (d'où ce nom de gelée royale). Il se produit donc chez la larve d'ouvrière, après le troisième jour, une véritable castration alimentaire qui empêche le développement normal des ovaires. La gelée royale contiendrait aussi une substance capable d'induire le développement ovarien mais cette substance est instable. Von RHeIN (I933) n'a jamais pu obtenir de reines par élevage in vitro parce qu'il se servait de gelée vieillie. WEAVER (I955) a réussi, au contraire, l'expérience en nourrissant les larves avec de la gelée royale fraîche prélevée toutes les deux heures. Nous verrons plus loin que le principe antibiotique contenu dans la gelée royale est capable, lui aussi de se modifier au cours du vieillissement de la substance. D'autre part GoILLOT (I957) a montré que les constantes physiques de la gelée variaient en fonction du temps et de la température de conservation. La larve, dans sa cellule, reçoit constamment de la gelée royale fraîche, toutes les deux ou trois minutes (KUWABARA, I947). On peut conclure de tout ceci que la gelée royale est une substance contenant des facteurs très labiles, et que la larve doit toujours avoir à sa disposition des provisions très fraîches, pour pouvoir se développer normalement.

La gelée royale est très acide, son $\mathrm{pH}$ se situe entre 3,6 et 3,7. Après neutralisation, elle fermente rapidement (VERGÉ, 1951). En effet, on 
y trouve de nombreuses spores de champignons et de levures qui sont inactivées, mais non détruites. Le $\mathrm{pH}$ de la gelée contribuerait à la stabiliser dans une certaine mesure.

La récolte et l'utilisation de cette matière par l'homme est très récente, mais la gelée royale est devenue rapidement très recherchée pour des raisons plus ou moins valables. Je n'entrerai pas dans les détails concernant ce sujet et le lecteur intéressé pourra se reporter à d'excellentes mises au point de Chauvin (1955, I956, 1958) concernant la récolte, la composition, l'action pharmacodynamique et l'utilisation de la gelée royale. Cependant avant d'aborder la question qui nous concerne ici, je pense qu'il n'est pas inutile d'exposer rapidement les propriétés physiques et la composition chimique de la gelée royale.

\section{PropriÉTÉs - COMPOSITION.}

La gelée royale a l'aspect d'une bouillie épaisse dont la couleur varie du blanc au jaune selon l'origine. Son goût est acide et son odeur légèrement phénolique, sa densité est environ de $\mathrm{I}, 4$. Cette substance se récolte entre le deuxième et le troisième jour, au moment du dépôt maximum et avant que la larve ne soit trop grosse. La gelée royale après sa récolte doit être mise à l'abri de l'air, de la lumière et de la chaleur. En effet, il peut s'y produire des oxydations, un brunissement de la matière, une perte d'eau et même des développements de moisissures. Au contact d'un métal, il se forme des sels qui peuvent être toxiques. La température de conservation habituelle est $\mathrm{de}+\mathrm{I}^{\circ} \mathrm{a}+3^{\circ} \mathrm{C}$.

Sa composition biochimique est relativement constante et les protéines y prédominent. Sa teneur en glucides et lipides est faible et seule cette dernière peut varier d'une manière importante. D'après Gontarski (I949), la gelée royale est chez l'abeille l'aliment larvaire le plus concentré, car elle contient moins d'eau que la nourriture des ouvrières et des mâles. D'après GorL_OT (1955) la gelée royale du troisième jour contient $70 \mathrm{p}$. Ioo d'eau. Le tableau XVIII reproduit les résultats d'analyse de la gelée royale par HAYDAK et Vivino (I943) en fonction de l'évolution de l'âge des larves ; il suffit à rendre compte de l'importance des substances azotées. Cet azote est présent sous forme de protéines, mais principalement sous forme d'acides aminés libres. Les glucides représentent $20 \mathrm{p}$. Ioo à $25 \mathrm{p}$. Ioo du poids sec et ce sont les mêmes sucres que ceux du miel. Les lipides extractibles par l'éther représentent de Io $p$. IOO à $I_{5} \mathrm{p}$. roo du poids sec et leur teneur varie beaucoup suivant 1'âge de la larve. Mais la caractéristique principale de la gelée royale réside dans sa richesse en vitamines (sauf les vitamines A et D qui sont peu ou pas représentées). L,e groupe B s'y trouve au complet et en abondance. La gelée royale est la source naturelle la plus viche en acide pantothénique. Il s'en trouve vingt fois plus que dans le pollen et l'on suppose 
que les jeunes abeilles le concentrent à partir de celui-ci. Les autres vitamines se rencontrent en pourcentages sensiblement égaux dans le pollen ou dans la gelée royale. Je terminerai cette revue en signalant la présence de quelques autres facteurs mal définis et souvent labiles, tel que celui qui agit sur le développement des ovaires. Pour plus de détails sur ces questions, il suffit de se reporter aux revues de Chauvin et de JOHANSSON (I958).

\section{TABLEAU XVIII}

Tableaux de la composition de la gelée royale d'après Haydak et Vivino (r943).

p. Ioo du poids frais de la gelée des reines.

\begin{tabular}{|c|c|c|c|c|c|c|c|}
\hline $\begin{array}{l}\text { Age en } \\
\text { jours }\end{array}$ & $\%$ eau & $\begin{array}{l}\text { Mat. } \\
\text { sèche }\end{array}$ & Azote & $\mid \begin{array}{c}\text { Protéines } \\
(\mathrm{N} \times 6,25)\end{array}$ & Graisses & Cendres & $\mathrm{pH}$ \\
\hline $1 \ldots$ & 65,37 & 34,63 & 2,24 & $\mathrm{I}_{4}$ & 2,63 & I,I9 & 4, I 5 \\
\hline $2 \ldots \ldots \ldots$ & $69, \mathrm{I} 7$ & 30,83 & 2,41 & I 5,06 & $\mathrm{I}, 73$ & $0,9 \mathrm{I}$ & 4,10 \\
\hline $3 \ldots \ldots \ldots \ldots$ & 69,88 & 30,12 & 2,44 & I 5,25 & 4,86 & 0,79 & 4,2 \\
\hline $4 \ldots \ldots \ldots$ & 69,7 & 30,3 & 2,24 & 14 & 5,68 & 0,70 & $4, \mathrm{I} 5$ \\
\hline $5 \ldots$ & 67,58 & 32,42 & 2,58 & $16, \mathrm{I}_{3}$ & 4,92 & 0,76 & 4,19 \\
\hline 6 operculé & $68,3^{2}$ & $3 \mathrm{I}, 68$ & 2,94 & I 8,38 & 3,99 & 0,75 & 4,15 \\
\hline
\end{tabular}

p. Ioo du poids sec de la gelée royale.

\begin{tabular}{|c|c|c|c|c|}
\hline Age & Azote & $\begin{array}{l}\text { P'rotéines } \\
(N \times 6,25)\end{array}$ & Graisses & Cendres \\
\hline 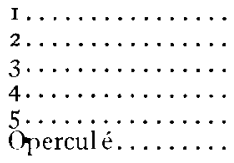 & $\begin{array}{l}6,47 \\
7,82 \\
8,1 \\
7,39 \\
7,93 \\
9,28\end{array}$ & $\begin{array}{l}40,43 \\
48,85 \\
50,63 \\
46,2 \\
49,75 \\
58, \text { OI }\end{array}$ & $\begin{array}{r}7,58 \\
5,61 \\
16,13 \\
18,74 \\
15,18 \\
\text { I } 2,59\end{array}$ & $\begin{array}{l}3,34 \\
2,95 \\
2,63 \\
2,3 \\
2,34 \\
2,37\end{array}$ \\
\hline
\end{tabular}

\section{$2^{\circ}$ Valeur antibiotique de la gelée royale.}

Après cette brève revue d'ensemble, nous allons examiner maintenant les quelques travaux ayant trait au principe antibactérien de la gelée royale.

En I939 Aввот'r remarque que la gelée ne se décompose pas à l'air et qu'elle s'y dessèche seulement. L'auteur pense que cela est dû en partie à la haute concentration en sucres et à un acide gras antiseptique. Mac ClESKey et MELAMPy (I938 et I939) montrent les premiers que la gelée royale possède des propriétés bactériostatiques et bactéricides. Le principe actif est insoluble dans l'eau, on peut l'entraîner par l'éther ou l'acétone qui laissent déposer après évaporation des cristaux doués de pouvoir bactéricide. Ce facteur est thermostable à $120^{\circ}$ pendant 20 minutes. 
D'après ces auteurs Escherichia coli et Salmonella typhi sont inhibées par une concentration à I/5o de gelée royale dans le bouillon et le Staphylococcus aureus avec I/IOO seulement. La température aurait une action importante sur le pouvoir germicide. Quand $\mathrm{I} \mathrm{ml}$ de bouillon était ajouté à $\mathrm{I} \mathrm{ml}$ de gelée diluée à $\mathbf{I} / 5$, à une température de $45^{\circ}$ les organismes étaient détruits en $I_{5}$ secondes, à une température de $25^{\circ}$, ils survivaient de ro à 30 minutes et à $5^{\circ}$, ils survivaient 2 jours. Les auteurs a méricains ont montré également que le pouvoir bactéricide baissait après neutralisation de l'acidité. VERGÉ (I95I) trouve que la gelée royale inhibe le développement de Escherichia coli et Bacillus prodigiosus. Mais, la croissance est à peu près normale après neutralisation du milieu avec tampon phosphaté. Vergé pense que l'acide qui lui a paru responsable du pouvoir antibiotique est en rapport avec le corps cristallisé recueilli par MAC Cleskey et Melampy. Hellev (1956) reprend les travaux sur le facteur antibiotique de la gelée royale. Il a notamment effectué ces essais avec de la gelée neutralisée préalablement et éprouvé les souches suivantes : un colibacille (non catalogué) et le Staphylococcus aureus (souche I ondres). Il a utilisé des gelées royales fraîches et lyophilisées. Les solutions n'ont pas été stérilisées de peur que le principe antibiotique soit thermolabile. Cet auteur a obtenu une action antibactérienne très nette sur le Staphylocoque. Au contraire, il n'a pas pu mettre en évidence un pouvoir antibiotique de la gelée neutralisée (dilution de I/20 à I/320) sur le colibacille, contrairement, dit-i1, aux résultats de MAC CLESKEY et MELAMPY. Mais ces derniers ont travaillé avec de la gelée non neutralisée et c'est, je pense, une différence suffisante entre les deux méthodes pour expliquer ces divergences. A certaines concentrations assez basses en gelée royale dans le milieu de culture (I/320) Hesleu trouve une stimulation de la croissance du Staphylocoque. Enfin il montre qu'une concentration du I/20 au I/80 est nécessaire pour empêcher tout développement ultérieur de la bactérie. Cet auteur pense, en conclusion que l'acidité de la gelée royale renforce ses propriétés antibactériennes mais qu'elle n'en est pas la cause essentielle et qu'il existe un facteur antimicrobien spécifique. Hinglais H., Hinglais M. et Gaurherie (I956) ne sont pas tout à fait d'accord avec HELLE: ; ils trouvent une action bactéricide de la gelée royale, mais pas de propriétés antibiotiques au sens habituel du terme : un contact de 6 heures du staplyylocoque avec la gelée royale tue définitivement les germes tandis que pour Proteus mlgaris, il suffit de I5 minutes de contact. En ce qui concerne les épreuves antibiotiques, une concentration élevée de Ioo $\mathrm{mg}$ par tube de culture $\left(2,5 \mathrm{~cm}^{3}\right.$ de milieu) n'a pas empêché la prolifération des deux souches étudiées. LANGLADE, Hinglais H., Hingla is M., (I957) ont étudié également la valeur microbicide de la gelée royale sur le Bacille de Koch. Ces essais ont montré que le principe bactéricide vis-à-vis de Mycobacterium tuberculosis est entière- 
ment soluble dans l'eau et dans l'alcool à $95^{\circ}$. L,es résidus insolubles sont inactifs. Dernièrement, MORELLINI (I958) a aussi essayé l'activité bactéricide de la gelée royale. Il a montré que la dose de $7 \mathrm{mg}$ par $\mathrm{cm}^{3}$ de milieu inhibait la croissance de Escherichia coli, Staphylococcus aureus, Bacillus megatherium et Proteus X I9. Mais l'action sur les levures est nulle ou faible, puisque cet auteur n'obtient qu'une inhibition partielle (avec $25 \mathrm{mg}$ de gelée par $\mathrm{cm}^{3}$ ) vis-à-vis de Saccharomyces cerevisiae.

L'ensemble des travaux effectués depuis 1939 sur le sujet montre de grandes divergences. C'est alors que j'ai commencé à effectuer mes essais sur la valeur antibiotique de la gelée royale. J'ai obtenu personnellement des résultats difficiles à interpréter, variant suivant les essais et d'un échantillon de gelée royale à un autre. L'analyse de tous ces essais préliminaires m'a donné une des clefs du problème : La gelée royale vieillit dans le temps en fonction de la température et la substance antibiotique présente n'est pas stable pas plus que celle qui préside au développement des ovaires des reines. Ceci posé et après deux ans de tâtonnements, j'ai pu éprouver la valeur antibiotique de la gelée royale et étudier son vieillissement.

\section{B. - Travaux personnels.}

\section{Io Matériel et méthodes.}

La gelée royale qui m'a servi pour les épreuves a été prélevée soit au laboratoire, soit chez des apiculteurs, mais, dans ce dernier cas, la récolte était contrôlée, ou pratiquée par nous-mêmes directement. La gelée était récoltée de 2 jours et demi à 3 jours après le greffage des cellules et placée immédiatement à la température convenable (variable suivant l'expérience donnée) soit sous forme de matière brute, soit en solutions diverses dans l'eau ou dans un solvant approprié. Nous avons déjà vu que le facteur antibiotique s'extrait bien par agitation à froid de la gelée dans l'eau ou dans l'alcool. Dans la plupart des cas, j'ai préféré opérer d'une autre manière de façon à entraîner le moins possible de corps non actifs (les sucres par exemple). J'ai extrait la gelée royale en la triturant au moyen de l'éther à froid. La solution obtenue est ensuite évaporée et le résidu repris par l'eau tiède. L,es résidus insolubles dans l'éther et dans l'eau sont inactifs. L'extrait de gelée royale est stable dans l'éther, mais son activité antibactérienne varie s'il est conservé en milieu aqueux, même à $\mathrm{o}^{\circ} \mathrm{C}$, comme je le montrerai par la suite. A la température ordinaire, en milieu aqueux, les modifications sont déjà sensibles au bout de quelques heures. Ia solution aqueuse incolore est ajoutée au milieu de culture synthétique habituel, suivant des doses croissantes. Les épreuves ont porté sur les 30 souches bactériennes du tablean I. Pour certains essais, sur le vieillissement par exemple, les épreuves ont été 
effectuées sur deux souches seulement, Bacillus subtilis souche Caron et Proteus vulgaris. Les milieux de culture ont été neutralisés ou non suivant les cas, mais je n'ai trouvé aucune différence significative pour une variation $d u p H$ du milieu. Le calcul des unités antibiotiques a été établi comme je l'ai déjà exposé dans les chapitres précédents. Le facteur antibiotique est thermostable et n'est pas détruit par l'autoclavage à $I 20^{\circ}$ pendant I5 minutes.

\section{$2^{\circ}$ Résultats.}

La gelée royale est liée à la vie de la jeune larve, et pourtant j'ai trouvé que les extraits de larve d'abeille sont dépourvus de substance antibiotique. La figure 33 montre la différence existant entre l'activité antibiotique de la gelée royale pure, des larves royales non lavées c'est-à-dire enrobées de gelée royale et des larves des différentes castes débarrassées de cette gelée par un lavage avant l'extraction. Il est net que les larves royales ne sont actives sur Bacillus subtilis que si elles apportent en même temps sur leur corps de la gelée royale. Il est curieux de noter que la gelée qui remplit leur intestin devient inactive dans les extraits, dès qu'elles l'ont ingérée. Le spectre d'activité de la gelée royale sur zo souches bactériennes est résumé sur la figure 34 . Les résultats sont les suivants :

- Action antibiotique très nette sur : Bacillus subtilis, Salmonella gallinarum, Escherichia coli B, Proteus X I9, Proteus vulgaris, Proteus V, Bacillus alvei, avec une action très importante sur les Proteus et Escherichia coli B.

- Action antibiotique, mais nécessitant une quantité d'extrait double de celle qui inhibe Bacillus subtilis, sur Salmonella pullorum ( 3 souches, $n^{0}$ I, 2 et 3), Salmonella type Dublin $n^{0} 7-54$, Escherichia coli (4 souches $\mathrm{n}^{\circ} \mathrm{I} 2, \mathrm{I} 3, \mathrm{I} 4, \mathrm{I} 5$, du tableau I), E. coli 36 et Bacillus lavvae.

- Enfin, action nulle sur Salmonella Dublin ( $\mathrm{n}^{\circ} 7$ et 8 du tableau I), Escherichia coli (5 souches $\mathrm{n}^{\circ}$ I0, II, I6, I7, I8 du tableau I), E. coli Bordet et Pseudomonas pyocyanea (No 25-26-27, du tableau I).

En conclusion, on peut dire que la gelée royale possède un facteur antibiotique très actif sur les Proteus et Escherichia coli B, mais dans l'ensemble il n'a que très peu ou pas d'action sur les Escherichia coli et le Pseudomonas pyocyanea. L'action sur les Salmonella pullorum est également assez faible. Nous verrons plus loin la comparaison de ces résultats avec ceux qui ont été acquis par les différents auteurs.

\section{$3^{0}$ Influence du vieillissement de la gelée royale.}

La gelée royale est une substance de composition assez stable, comme nous l'avons vu, mais qui a cependant tendance à se modifier sous certains égards au cours du temps. Par exemple, Drxon et SHUEL 


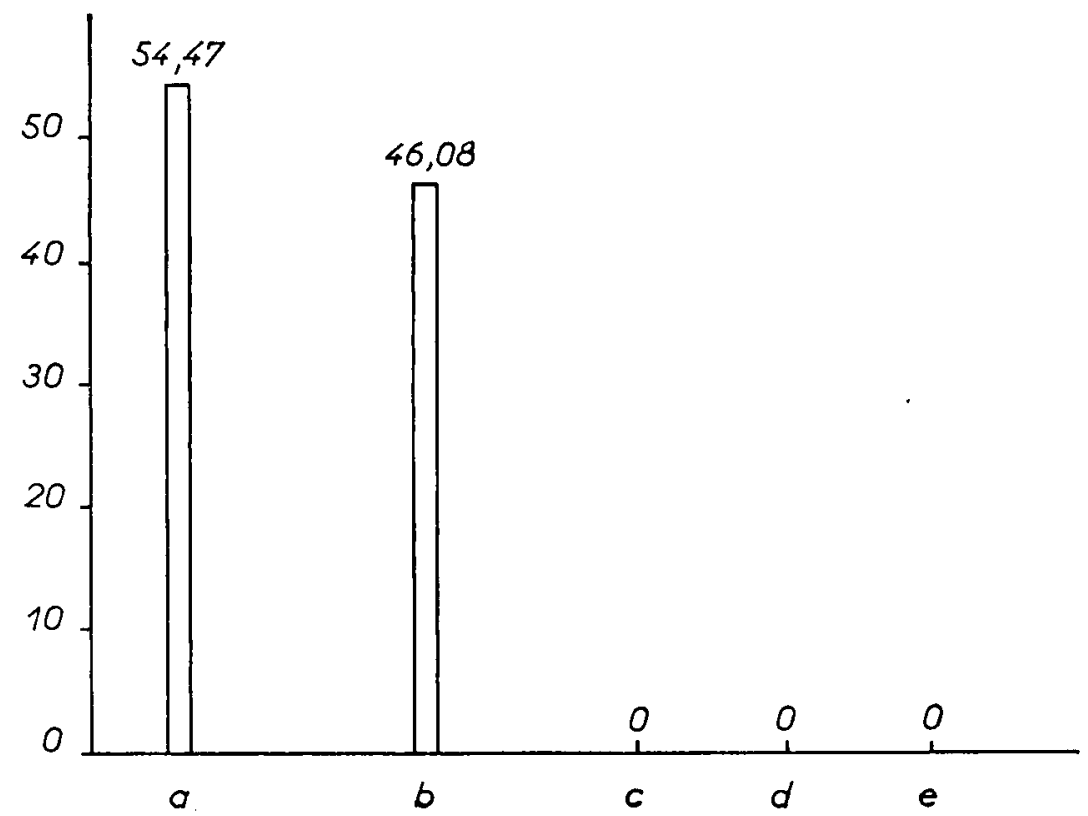

Fig. 33. - Comparaison de l'action antibiotique de la gelée royale et des larves (épreuve B. subtilis). $a$ : gelée royale pure.

$b$ : larves royales avec gelée (non lavées).

$c$ : larves royales sans gelée (lavées).

$d$ : larves d'ouvrières (lavées).

$e$ : larves de mâles (lavées).

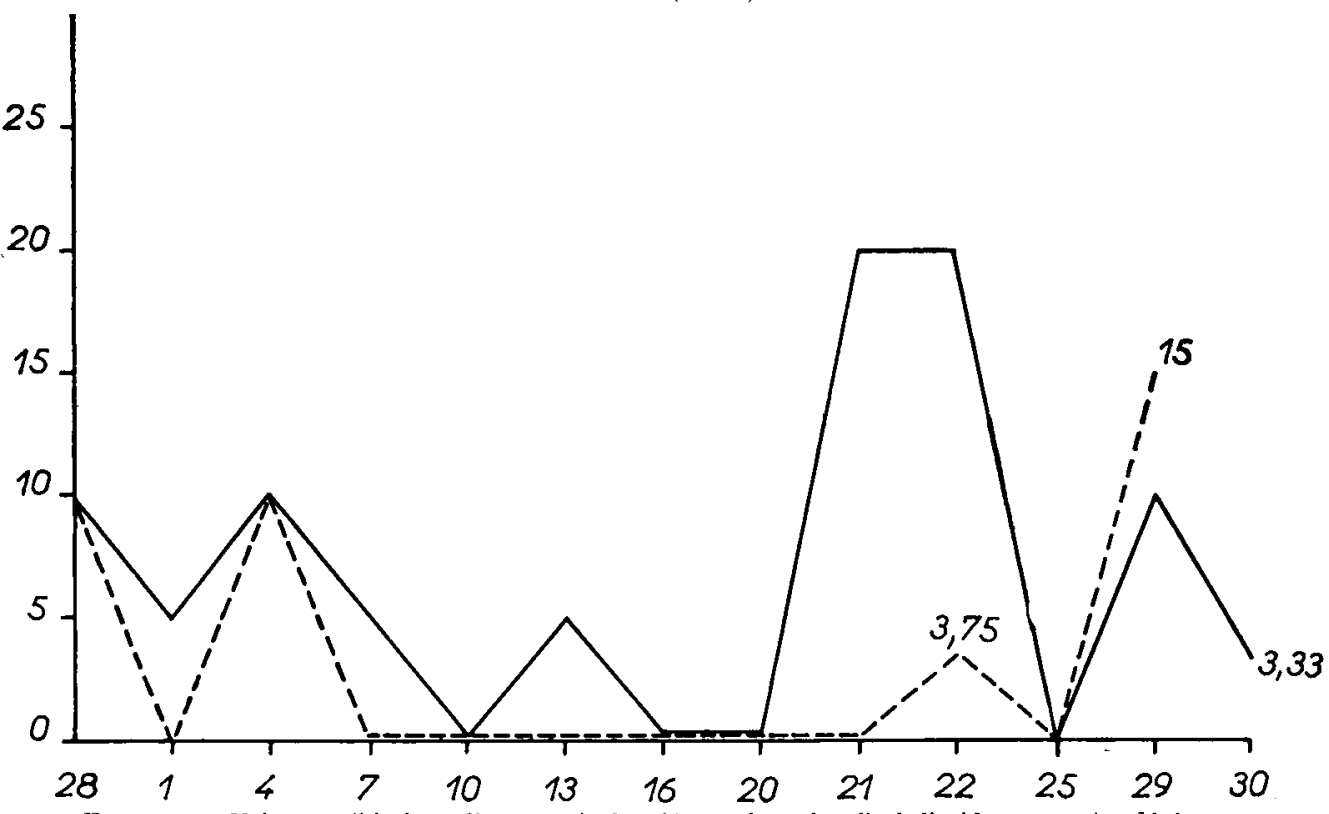

FIG. 34. - Valeur antibiotique d'un extrait de gelée royale et de celle de l'acide ro-oxy- $\mathbf{2}$ décénoique de synthèse (en abscisse les numéros des souches éprouvées).

- - extrait gelée rovale.

.. extrait de l'acide de synthése. 
(I958) trouvent que l'absorption d'oxygène mesurée au ludion ne peut être mise en évidence qu'avec de la gelée royale très frâiche. Des modifications progressives de la gelée royale ont été trouvées par Goiliot (I957) au cours de mesures électriques sur des échantillons que j'ai moimême éprouvés du point de vue de leur activité antibiotique. Rappelons d'autre part les expériences de WEAVER (I955) qui ne peut élever des reines in vitro en obtenant le plein développement des ovaires si la gelée n'est pas renouvelée toutes les 2 heures. Enfin, CHauvin (I957) montre que les solutions fraîchement préparées de gelée royale ne sont pas hyper-

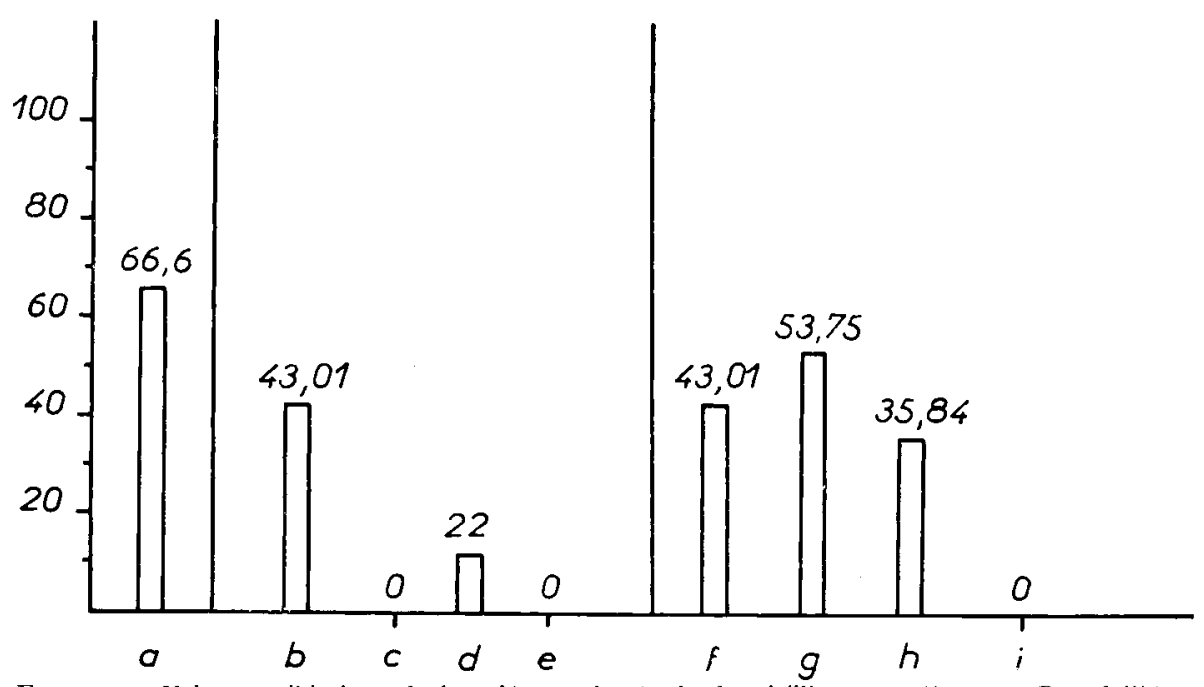

Frg. 35. - Valeur antibiotique de la grelée rovale, étucle du vieillissement (épreuve B. subilitis).

$$
\begin{aligned}
& a \text { : gelée rovale, l'émoin conservé en solution éthérée à oo. } \\
& b \text {-c-d-e : ieléc royale en solution aqueuse conservéc à } 20^{\circ}-25^{\circ} \text {. } \\
& f-g-h-i-: \text { ielée rovale en solution aqueuse conservée di } o^{\prime \prime} \text {. } \\
& b \text { et } f \text { : Solutions fraiches. } \\
& c \text { : apres } 2+\text { heures. } \\
& d \text { : aprés } 48 \text { heures. } \\
& e: \text { après } 72 \text { heures. } \\
& g \text { : après I I jours. } \\
& h \text { : apres if jours. } \\
& i \text { : apres Ig jours. }
\end{aligned}
$$

glycémiantes pour la souris, mais le deviennent après un "vieillissement" de quelques jours au réfrigérateur. En pleine concordance avec ces faits, la valeur antibiotique de la gelée royale ou de ses solutions aqueuses varie beaucoup, bien qu'assez irrégulièrement avec le vieillissement $d u$ produit. La figure 35 rend compte de l'activité de l'extrait de gelée sur $B$. subtilis souche Caron, en fonction du temps, à certaines températures de stockage. Je signale tout d'abord que l'extrait de gelée royale en solution éthérée, conservé à $0^{\circ}$, garde sa valeur pendant plusieurs mois. Seules, se modifient la gelée royale brute et les solutions aquenses. A la température ordinaire du laboratoire, lorsqu'on fait des solutions de gelée d'âges différents et 
qu'on les éprouve aussitôt sur Bacillus subtilis, on constate que la gelée n'est active que le premier jour de sa récolte. Elle est inactive le lendemain; le $3^{\mathrm{e}}$ jour apparaît une faible activité (moitié moins que le jour de la récolte) qui disparaît complètement I jour plus tard. Dans le cas d'une solution aqueuse, non plus essayée aussitôt, mais conservée à oo, l'action antibiotique qui correspond à 43 unités subtilis le premier jour, passe à 53 unités le I I jour, à 35 le $\mathrm{I} 5^{\mathrm{e}}$, pour s'annuler définitivement à partir du I $^{\mathrm{e}}$ jour. D'autre part, si la solution de gelée royale conservée à oo est placée à $20^{\circ}-25^{\circ}$, l'action antibiotique disparaît en 24 heures pour les Io souches bactériennes ( $3 E$. coli, S. type Dublin, E. coli Bordet, Proteus, 2 Salmonellae, $P$. pyocyanea et $B$. subtilis et elle est très atténuée pour Salmonella pullorum, Salmonella gallinarum et Proteus V.). En 48 heures, il n'y a plus qu'une faible action sur subtilis. Au bout de 72 heures, l'action est définitivement nulle sur toutes les souches essayées. Il est donc très net que l'élévation de température accélère le processus du vieillissement de la gelée royale. L'atmosphère si particulière de la ruche devrait faire varier constamment le taux d'antibiotique dans la gelée royale; or, les échantillons retirés de la ruche ont la même valeur moyenne : sans doute parce que la gelée royale est dégorgée par les abeilles nourrices toutes les 2 ou 3 minutes (KUWABARA, I947), si bien qu'on peut la considérer comme perpétuellement fraîche. Des expériences en parallèle avec GoIllot ne nous ont pas permis de trouver de rapport fixe entre la variation des mesures physiques et la variation de la valeur antibiotique.

Quelles transformations biochimiques se produisent dans la gelée royale? Il s'agit peut-être de phénomènes d'oxydation ou d'hydrolyse, mais nous ne disposons encore ici que d'hypothèses. Ces modifications profondes expliquent certainement les différences de résultats obtenus par les chercheurs qui ont travaillé pour la plupart sur des gelées d'âge et d'état de conservation non défini. Je pense qu'il est du plus grand intérêt dans les essais futurs de tenir compte de cet état de fait, plus important même que l'origine géographique des gelées. Si l'on veut obtenir une substance antibiotique stable, il faudrait extraire celle-ci de la gelée au moment de sa récolte et la conserver ensuite en phase éthérée ce qui éviterait toute modification ultérieure.

Cette influence du vieillissement fait penser aux modifications que le pollen récolté par les abeilles subit à l'intérieur de la ruche. Avant d'en finir avec ce problème, il faut cependant noter que cette substance si sensible à une élévation de température de $20^{\circ}$ à $30^{\circ}$ au-dessus de $0^{\circ}$ pendant quelques heures, résiste à $120^{\circ}$ (mélangée au milieu de culture) pendant I5 à 20 minutes lors de l'autoclavage. D'autre part de la gelée royale mise à $-35^{\circ} \mathrm{C}$ dès sa récolte ne se modifie pas pendant plusieurs mois. 


\section{$4^{\mathrm{o}}$ Fractionnement de la gelée royale.}

Dans presque tous les essais que j'ai pratiqués sur la gelée royale, I $\mathrm{cm}^{3}$ de la solution aqueuse finale à éprouver correspondait toujours à I $g$ de gelée fraîche, soit $0,333 \mathrm{~g}$ d'extrait sec ou encore o,ozo $g$ d'acides totaux. La dilution limite moyenne pour $B$. subtilis étant égale à $0,015 \mathrm{~g}$ d'acides $\left(0,5 \mathrm{~cm}^{3}\right.$ de solution). C'est cette fraction acide qui est active au point de vue antibiotique dans la gelée. D'autre part, Butenandt et Rembold (I957) ont isolé de la gelée royale l'acide ro-oxy $\Delta_{2}$ décénoïque que $\mathrm{j}$ 'ai pu étudier grâce à l'obligeance du professeur BUTENANDT. Le peu de matière confiée a été mélangée à Io $\mathrm{cm}^{3}$ de milieu synthétique et le tout autoclavé à $120^{\circ}$. Cette substance a inhibé complètement le développement de Bacillus subtilis souche Caron. J'ai repris ces recherches grâce à un supplément de la même substance obtenue ultérieurement pure à $83 \mathrm{p}$. Ioo environ et en quantité suffisante pour éprouver une douzaine de bactéries diverses. I a figure 34 montre les résultats comparés à ceux qui ont été obtenus au moyen des extraits de gelée pure. Les deux substances ont été arbitrairement ramenées à la valeur ro pour leur action sur Bacillus subtilis souche Caron, et les autres valeurs calculées en fonction de celle-ci. L'acide de synthèse est actif sur Bacillus subtilis avec une dose de $\mathrm{I} 8 \mathrm{mg}$ dans Io $\mathrm{cm}^{3}$. Si l'on regarde de près les deux courbes, on constate que dans les deux cas, il n'y a aucune action sur plusieurs Escherichia coli et Pseudomonas pyocyanea, il existe une activité voisine pour Salmonella gallinarum et Bacillus alvei. Mais,je trouve de grandes différences sur d'autres points, l'extrait de gelée royale est très actif sur $E$. coli B et sur Proteus vulgaris, tandis que 1'acide de synthèse est sans action sur la première souche et très peu actif sur la seconde. Il n'existe donc pas un parallélisme d'action des deux substances sur l'ensemble des souches éprouvées mais simplement des ressemblances. On ne peut déduire de ces résultats que 1'acide ıo-oxy $\Delta_{2}$ décénoïque est le seul facteur antibiotique présent dans la gelée royale. Il existe probablement plusieurs corps actifs.

\section{$5^{\circ}$ Actions d'autres facteurs présents dans la gelée royale.}

La gelée royale n'est pas antifongique. En effet, de la gelée abandonnée à 1'air et même à $o^{\circ}$ au réfrigérateur se couvre parfois de Penicillium. Les solutions aqueuses de gelée royale fermentent rapidement à la température ordinaire. Morflinin a d'ailleurs montré que Saccharomyces cerevisiae pouvait encore se développer dans un milieu contenant $25 \mathrm{mg}$ de gelée par $\mathrm{cm}^{3}$. J'ai moi-même constaté que des champignons divers se développaient sur un milieu synthétique additionné d'extrait éthéré de gelée royale (l'extrait de I g de gelée dans Io $\mathrm{cm}^{3}$ de milieu). 
D'autre part, j'ai étudié la valeur antigerminative de la gelée royale fraîche sur les pollens. L'essai de germination a été effectué avec du pollen de Papaver rhaeas, fraîchement récolté à cet effet. Un milieu gélosé et sucré à Io $\mathrm{p}$. IOO additionné de l'extrait de I g de gelée ( $5_{5} \mathrm{mg}$ d'acides totaux) pour Io $\mathrm{cm}^{3}$ empêche totalement la germination des grains de pollen. Le même milieu avec une dose 2 fois moindre d'extrait permet un début de germination d'environ Io $\mathrm{p}$. Ioo des grains de pollen qui avortent ensuite très rapidement. I,es pollens sur milieu témoin ont un développement norma1. D'ailleurs, Maurizio (1958) avait déjà montré que la nourriture larvaire est inhibitrice de la germination du pollen. Ce facteur subsiste après chauffage et ne dépend pas du $\mathrm{pH}$. I.ouveaux et Chauvin (I956) lors de travaux sur l'analyse pollinique de la gelée royale avaient déjà remarqué la présence dans cette substance de pollens ayant un aspect normal, comme ceux qui sont présents dans le miel, et d'autres grains de pollen altérés et détruits en partie, Mais, ils n'ont jamais trouvé de pollens germés dans la gelée royale. Les auteurs ont montré également que la gelée contenait des spores de champignon, des débris de mycélium, des levures, mais ils ne signalent pas la présence de corps bactériens. En ce qui concerne l'action sur la germination des pollens, j'ai pu préciser que le facteur antigerminatif passe dans l'éther et dans l'eau et qu'il ne se différencie pas du principe antibiotique de la gelée royale.

En résumé, il existe dans la gelée royale un puissant inhibiteur de la germination des grains de pollens, mais pas de principe antifongique.

\section{$6^{\circ}$ Comparaison entre les résultats obtenus et ceux des autres auteurs.}

J'ai résumé sur le tableau XIX mes résultats et ceux obtenus par les différents auteurs. On peut tirer malgré les grandes divergences quelques conclusions communes.

a) L $\mathrm{e}$ pH n'influence pas l'action du facteur antibactérien présent dans la gelée (d'après les travaux récents). I,es premiers auteurs cependant ne sont pas de cet avis.

b) I e principe actif de la gelée royale est extractible à froid par l'eau, l'alcool, l'acétone et l'éther.

c) I a valeur bactéricide de la gelée royale est admise par tous.

d) I a valeur antibiotique au sens habituel du terme est discutée, et refusée par Hingiais (H.) et Hinglais (M.), Gautherie et Ianglade.

e) I a gelée n'est pas antifongique (MORELIINI et LAVIE).

f) J'ai trouvé que le principe antibiotique de la gelée est thermostable (I5 minutes à $120^{\circ}$ ) et HriLlEu (I958) vient de montrer la même chose.

g) En ce qui concerne le vieillissement, il a été entrevu par Mac 


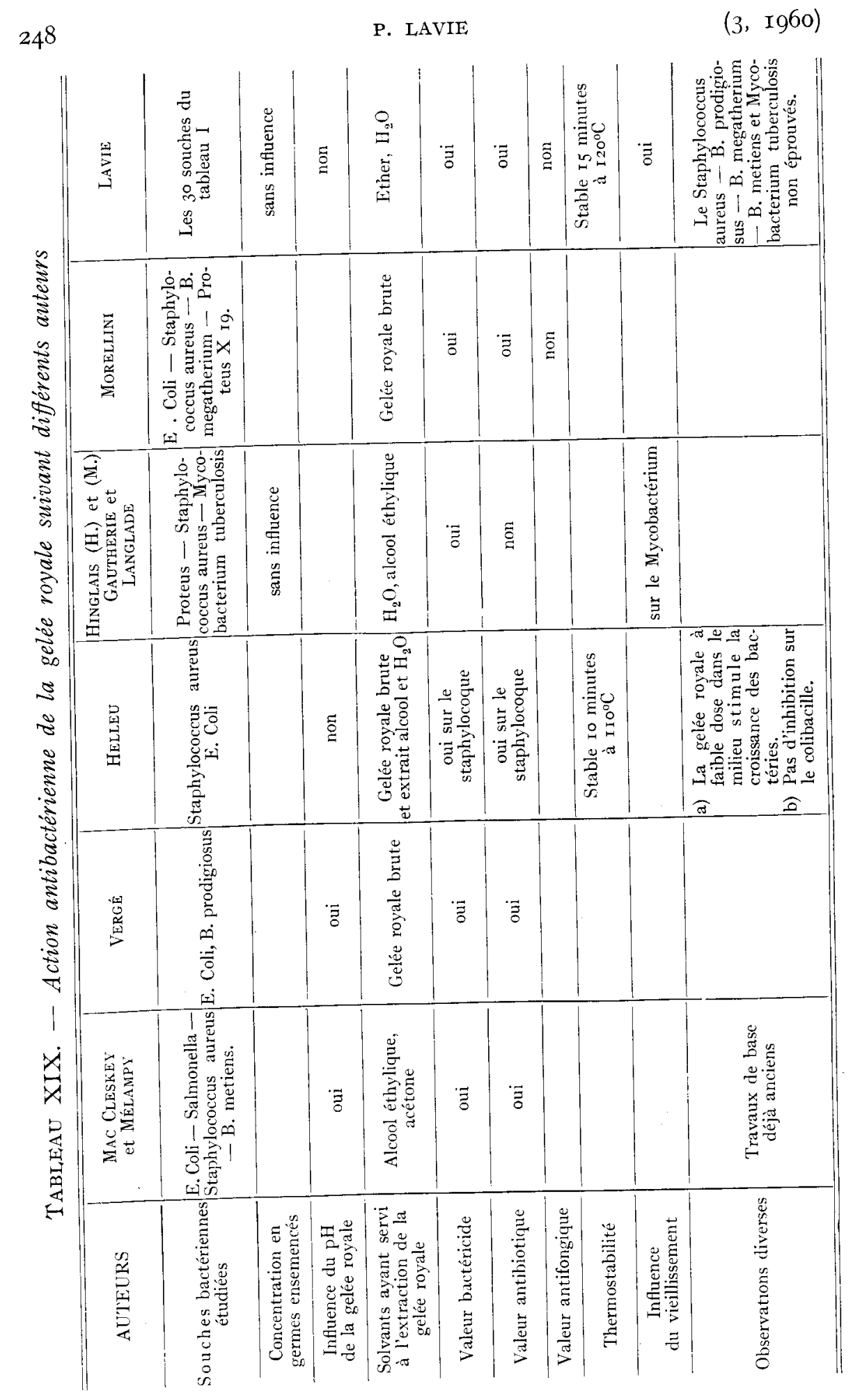


Cleskey et Melampy et je l'ai étudié en fonction de la température. Au contraire, LANGlade, Hinglais (H.) et Hinglais (M.) trouvent sur le bacille de Koch des résultats semblables avec des gelées d'origines diverses conservées de 8 jours à plusieurs mois, dont l'une conservée à $+4^{0}$ pendant 8 mois. Je persiste à penser malgré tout qu'il existe dans la gelée royale un facteur antibactérien très labile et qui varie d'activité avec 1'âge de cette substance. Ce qui se trouve d'ailleurs corroboré comme nous l'avons vu par les autres transformations physiques et biochimiques de la gelée royale. Enfin, les auteurs précités n'ont pas testé de la gelée royale de moins de huit jours ce qui explique peut-être nos divergences.

Parmi les souches étudiées par les différents auteurs et par nousmêmes se retrouvent: Escherichia coli, des Salmonella, Proteus. Le choix des Escherichia coli n'était peut-être pas très judicieux, car j'ai montré que les extraits de gelée étaient peu ou pas actifs sur ces souches. Cependant MaUrizio et GUbler (I95I) ont montré qu'un extrait de gelée par le chloroforme (chauffé ou non) était actif sur Escherichia coli. En revanche, j'ai trouvé une action antibiotique très nette sur Proteus (7,5 $\mathrm{mg}$ par ro $\mathrm{cm}^{3}$ de milieu) de même que Morelírini. Or, Hinglais et alt. ne trouvent aucune activité sur cette bactérie avec $40 \mathrm{mg}$ d'extrait par $\mathrm{cm}^{3}$ de milieu. En ce qui concerne la valeur antigerminative de la gelée, les résultats que $j$ 'ai obtenus et ceux de Maurizio concordent parfaitement et ils confirment les observations d'autres auteurs.

\section{C. - Conclusion du chapitre V.}

$I^{\circ}$ La gelée royale possède des propriétés antibactériennes et les travaux récents ont montré que la valeur très basse de son $\mathrm{pH}$ n'entrait pas uniquement en jeu dans ce mécanisme. Cependant, ce principe antibactérien est contenu dans la fraction acide de la gelée royale. Ces résultats expliquent quelques faits connus antérieurement, à savoir que la gelée royale pure malgré sa richesse en substances nutritives et en facteurs de croissance donne souvent sur des milieux ordinaires de culture des ensemencements stériles, même si elle est prélevée sans précaution d'asepsie. La gelée royale abandonnée à l'air ne se putréfie pas, mais elle se dessèche et elle peut se conserver ainsi très longtemps. D'après VERGÉ (I95I), si on neutralise une solution de gelée royale, il y a possibilité de fermentation, car les levures s'y développent alors. Mais nous avons vu que la fraction antibiotique ne s'est pas révélée antifongique. Ceci explique également le développement assez fréquent des Penicillium sur de la gelée pure même au réfrigérateur (entre $+\mathrm{I}^{0}$ et $\left.+4^{0}\right)$. D'autre part, l'action antibiotique de la gelée royale et du pollen explique en partie la stérilité du tube digestif des larves et des abeilles naissantes (BRUCF. WHITE). 
$2^{0}$ La gelée royale est une substance complexe dont de nombreux principes sont labiles. En ce qui concerne son antibiotique, j'ai montré que le "vieillissement " était important principalement dans les premiers jours à température ordinaire. D’après GoILLOT (I957), la gelée rovale pure est stable au point de vue conductivité électrique pendant 20 heures maximum pour une température de $0^{\circ}$ à $+30^{\circ} \mathrm{C}$. Ensuite, intervient une période variable, mais d'environ 400 heures pendant laquelle la gelée "vieillit intensément "suivie d'une période de "vieillissement " très lent où la conductivité croissante tend vers une limite (jusqu'au huitième mois). Cependant, l'activité antibiotique ne suit pas une courbe parallèle à celle de la conductivité électrique et je ne puis préciser actuellement pourquoi le facteur antibiotique réagit d'une manière si complexe au cours du temps.

$3^{\circ}$ L'action de la gelée royale sur les souches pathogènes envers l'abeille est faible, mais toujours présente. L'action de l'extrait éthéré de gelée royale est faible vis-à-vis de Bacillus alvei; l'inhibition est obtenue avec l'extrait de $\mathrm{I} g$ de gelée fraîche dans ro $\mathrm{cm}^{3}$ de milieu ( $\mathrm{pH}$ ajusté). Cette activité est encore plus faible vis-à-vis de Bacillus larvae puisque l'inhibition n'est obtenue qu'avec l'extrait de $3 \mathrm{~g}$ de gelée fraîche dans les mêmes conditions. Cependant, la gelée a un effet retardateur sur Bacillus larvae si l'on se place en dessous de la dilution d'inhibition; avec l'extrait de $2 \mathrm{~g}$ de gelée, on obtient un retard de 48 heures dans le développement du bacille. En résumé, l'action antibiotique de la gelée est très faible envers les souches pathogènes de la colonie d'abeilles. Cependant étant donné la fréquence de distribution de cette substance aux larves, on peut admettre que, dans une certaine mesure, la gelée royale joue un rôle dans la défense chimique contre les infections de la ruche. L'acidité de la nourriture des larves jeunes joue un rôle important dans l'étiologie des loques. Matuka et Topornik (I952) ont montré que dans un milieu à $\mathrm{pH} 3,5$ les formes végétatives de Bacillus larvae périssent en 5 heures et celles de Bacillus alvei entre une demi-heure à I heure. Toutes ces observations peuvent expliquer les nombreux échecs d'infection artificielle $\mathrm{du}$ couvain par les formes végétatives des germes qui provoquent les loques. D'autre part, ces faits expliqueraient aussi la réussite des infections sur un jeune couvain privé de nourriture. KRAsIKova (I955) a montré, d'autre part, la valeur antibactérienne de la gelée royale vis-àvis des souches responsables de la loque européenne. D'après cet auteur, la gelée royale et la nourriture des larves d'abeilles des deux premiers jours ont une valeur antibiotique vis-à-vis de Bacillus alvei et Streptococcus apis. Enfin, la gelée royale conservée 20 à 25 jours à température ordinaire serait toujours active sur les deux souches précitées. 
$4^{\circ}$ Rapport de la gelée royale avec les autres produits de la ruche.

I a gelée royale est une matière azotée produite par les glandes pharyngiennes des jeunes abeilles et son origine indirecte se trouve nécessairement dans la consommation du pollen. Il était raisonnable de penser que la substance antibiotique du pollen pouvait être voisine de celle qui est présente dans la gelée : or, l'expérience a montré qu'il n'en était rien. L'examen de la figure 26 permet de se rendre compte de ces différences. L'action du pollen est toujours plus importante que l'action de la gelée par rapport à l'action sur Bacillus subtilis pris comme unité. I,e pollen agit comme un très puissant inhibiteur sur les Salmonellae et sur les Proteus (Io fois plus que sur Bacillus subtilis) tandis que l'extrait de gelée a une action faible sur les Salmonellae et seulement double de celle sur Bacillus subtilis en ce qui concerne Proteus vulgaris. L'activité des deux substances est plus voisine sur les Escherichia coli sauf pour Escherichia coli B. De même 1'inhibition sur Bacillus larvae et Bacillus alvei est parallèle. Au contraire l'extrait de pollen agit sur Pseudomonas pyocyanea (même action que sur Bacillus subtilis) tandis que la gelée royale n'inhibe pas la croissance de cette bactérie. On peut conclure de ces résultats que les deux facteurs antibiotiques sont distincts. L'examen des figures 20 , 26 et 27 ne m'a pas permis non plus de relier l'action antibiotique de la gelée à celle des autres produits de la ruche ou de 1'abeille. La cire, qui est également une sécrétion de l'insecte, ne possède pas la même action inhibitrice sur les souches éprouvées. Malgré les écarts trouvés entre l'activité antibactérienne du pollen et de la gelée royale, rien ne prouve toutefois que ces facteurs soient très différents du point de vue chimique et qu'ils n'aient pas une origine commune. Le principe antibiotique extrait de l'abeille est aussi très différent de celui de la gelée royale. Cette dernière constatation infirmerait l'hypothèse selon laquelle l'antibiotique de l'abeille aurait pour origine la sécrétion des glandes pharyngiennes. Mais rien ne prouve que cette sécrétion glandulaire ne subit pas une transformation importante dès qu'elle parvient à l'extérieur au contact de l'air, ce qui peut modifier totalement ses propriétés.

$5^{\circ}$ La production de gelée royale dans la ruche est constante lors de la présence du jeune couvain dans la ruche et équilibrée par rapport à son extension.

Il se crée un équilibre entre le développement ou la régression du couvain et la production de gelée par les abeilles. Le facteur antibiotique sans doute très intéressant pour les jeunes larves, est toujours présent malgré son instabilité, à cause du dégorgement toujours renouvelé de gelée par les ouvrières. Ce principe antibiotique assez faible auquel s'ajoute l'acidité de la substance royale contribue cependant puissam- 
ment à maintenir le jeune couvain à 1'abri des épizooties par sa présence constante. Si la nourriture royale vient à manquer d'une façon naturelle ou artificielle, nous savons que l'infection par les souches pathogènes devient alors très facile à réaliser. Ce cas peut se présenter lors d'un développement important de la ponte et de l'élevage correspondant à une déficience inopinée des substances azotées dans la colonie. La sécrétion de la gelée royale est un phénomène social réglé par l'ensemble de l'essaim et non pas relevant du seul hasard. Si cet équilibre est rompu pour des causes diverses, il s'ensuit de graves inconvénients pour la ruche tout entière, exactement comme nous pouvons le constater dans l'organisme d'un être supérieur. 


\section{Chapitre: VI}

\section{ACTIONS DIVERSES DUES AUX SUBSTANCES PRÉSENTES DANS LA COLONIE D'ABEILLES}

Nous avons vu, au cours de l'étude de chacun des produits de la ruche, 1'action de certains sur la germination du pollen ou des graines ou sur le développement des champignons. J'ai voulu reconsidérer dans ce chapitre ces diverses actions pour en rechercher les rapports entre eux et vis-à-vis de l'action antibiotique. Le tableau XX résume l'ensemble de ces essais que je vais analyser dans les paragraphes suivants. Je signalerai ensuite assez rapidement des travaux ayant trait à la valeur antibiotique de Bacillus larvae, agent pathogène du couvain d'Apis mellifica.

\section{$\mathrm{I}^{\mathrm{O}}$ Valeur antigerminative des produits de la ruche sur le pollen.}

J'ai déjà montré que certains produits de la ruche sont inhibiteurs de la germination des pollens et des graines.

En ce qui concerne l'inhibition de la germination des pollens stockés à l'intérieur de la ruche les travaux de MaURizio ont montré de manière très nette l'influence $d u$ miel, de la nourriture larvaire, de l'antibiotique de l'abeille et de la sécrétion des glandes pharyngiennes. J'ai contrôlé ces résultats et je les ai complétés. J'ai trouvé que les extraits de propolis et de gelée royale (prélevée dans les cellules au $3^{\mathrm{e}}$ jour) étaient également inhibiteurs de la germination pollinique (la propolis très fortement). Un extrait de pollens récoltés par les abeilles (et renfermant en plus du pollen nombre de substances diverses) est faiblement inhibiteur pour la germination d'un pollen. D'après ces recherches (voir tableau $\mathrm{XX}$ ), c'est la propolis qui serait la substance la plus active, la gelée royale le miel, 1'extrait d'abeille l'étant beaucoup moins. Quant au pollen en pelotes, son action est très faible, sans doute à cause de la concentration trop peu importante en sécrétion salivaire ou en nectar. Un extrait de pollen stocké (même en dehors de la ruche) ayant subi diverses transformations serait sans doute plus actif. On voit l'importance de cette action antigerminative sur les grains de pollens, car elle permet à l'abeille de conserver le pollen à l'intérieur de la ruche sans que celui-ci en germant ne fasse éclater les cellules. I1 est évident que sans ces substances inhibitrices, 
voisines ou identiques aux facteurs antibiotiques, le pollen germerait très bien dans l'atmosphère favorable de la ruche. Or, nous savons qu'après 5 jours d'emmagasinage, il a perdu ses facultés germinatives.

\section{$2^{\circ}$ Valeur antigerminative des produits de la ruche sur les graines et les tubercules.}

I.es substances inhibitrices de la germination des graines et des tubercules sont en général des corps voisins du point de vue chimique de certains antibiotiques (CHOUARD, I956). On y trouve principalement des acides organiques, des aldéhydes, des alcaloïdes, des poisons respiratoires, des anti-auxines, des toxiques et des lactones non saturées (coumarine). Le terreau formé par la décomposition des feuilles contient des lactones non saturées qui empêchent la germination des graines d'autres espèces végétales. Ces corps n'inhibent pas la plante vivante qui les contient, car ils se trouvent souvent liés dans des combinaisons hétérosidiques qui bloquent l'activité inhibitrice. D'autre part, nous avons vu que l'iridomyrmécine est un corps à fonction lactone non saturée, que les aldéhydes sont présents dans le Populus nigra et dans l'antibiotique extrait de Melasoma populi. Il est établi également que les graines stockées par les fourmis à l'intérieur de leur nid ne germent pas tant qu'elles ne sont pas enlevées de ce milieu et qu'il ne se développe que très peu de végétation sur le dôme des fourmilières. C'est pourquoi il m'a semblé intéressant de voir si les produits de la ruche ne possédaient pas en plus de leur action antibiotique sur les bactéries et de leur valeur antigerminative sur les pollens une action inhibitrice sur la germination des graines et des tubercules.

Dans une première expérience, j'ai placé des tubercules de pommes de terre en état de germer (et après enlèvement de tous les germes) pendant trois semaines dans une ruche peuplée, les abeilles ayant librement accès aux tubercules. Un lot de pommes de terre témoins avait été placé dans une étuve ayant les mêmes conditions de milieu que la ruche (température, humidité) sauf la présence des abeilles. Au bout de ro jours les témoins commencent à germer alors que les pommes de terre dans la ruche ne présentent aucun germe. Les abeilles ont nettoyé ces pommes de terre et les ont enduites d'une légère couche de propolis (semble-t-il). Après 2 I jours dans la ruche pas un seul germe ne s'est développé sur les 8 tubercules en expérience, tandis que dans l'étuve 9 tubercules sur Io ont très bien germé. L'expérience a continué de la manière suivante : les tubercules sortis de la ruche ont été séparés en deux lots et plantés dans des pots pour voir si la substance antigerminative conservait ou non son pouvoir une fois le matériel soustrait à l'action de l'abeille. Le premier lot est planté tel quel sans aucun lavage. I,e deuxième lot avant 
d'être mis en terre, subit un lavage à l'alcool et un rinçage à l'eau dans le but d'éliminer soit la propolis, soit l'antibiotique de l'abeille à la surface des tubercules. Un troisième lot comprenait des pommes de terre identiques (dégermées), mais n'ayant pas séjourné dans la ruche. Après vingt jours les trois lots sont déterrés et examinés. Dans le lot $\mathrm{n}^{\mathbf{0}} 2$ (tubercules lavés à l'alcool) et le lot $n^{\circ} 3$ (témoin) les pommes de terre sont toutes germées, tandis que le $\operatorname{lot}^{\circ}{ }^{\circ}$ I n'a germé en aucune façon. Je puis conclure que dans la ruche les abeilles ajoutent une substance alcoolo-soluble qui inhibe la germination des pommes de terre, mais qui n'est pas germicide. A partir de ces résultats j'ai essayé vainement plusieurs fois de reproduire artificiellement l'inhibition à l'étuve, hors de la présence des abeilles. J'ai enduit d'extrait d'abeilles et d'extrait de propolis des pommes de terre qui ont été portées ensuite dans les conditions du milieu de la ruche. Tous ces essais ont échoué : les pommes de terre traitées ne subissent aucune inhibition et se développent comme les témoins. Jusqu'à présent je n'ai pas trouvé d'explication à ces échecs qui d'ailleurs ne cadrent pas comme nous allons le voir avec les expériences qui ont suivi où j'ai éprouvé les différents extraits des produits de la ruche sur la germination du riz.

\section{Tableau XX}

Modes d'actions divers des produits de la ruche.

\begin{tabular}{|c|c|c|c|c|}
\hline Extraits éprouvés & $\begin{array}{c}\text { Action } \\
\text { antigerminative } \\
\text { sur le pollen frais }\end{array}$ & $\mid \begin{array}{c}\text { Action } \\
\text { antigerminative } \\
\text { sur graines (de riz) }\end{array}$ & $\begin{array}{c}\text { Action } \\
\text { antifongique }\end{array}$ & $\begin{array}{l}\text { Action } \\
\text { antibactérienne }\end{array}$ \\
\hline Abeille & + & +- & 一 & + \\
\hline Miel & + & $+t$ & -+ & + \\
\hline Pollen en pelotes & +- & +- & -- & + \\
\hline Propolis & ++ & ++ & ++ & + \\
\hline Gelée royale & + & +- & - & + \\
\hline Cire & non essayé & + & - & + \\
\hline $\begin{array}{l}\text { Venin d'abeille } \\
\text { (Lemay) }\end{array}$ & non essayé & $\begin{array}{c}+ \\
\text { (graines non } \\
\text { précisées) }\end{array}$ & non essayé & + \\
\hline
\end{tabular}

I1 est difficile de placer dans une ruche de petites graines, car les abeilles qui nettoient sans cesse leur demeure les évacueraient facilement. J'ai donc opéré d'une autre manière. J'ai testé la valeur des extraits (en phase aqueuse) d'abeille, de propolis, de cire, de miel, de gelée royale et de pollen en pelotes sur la germination de graines de riz placées en boîtes de Pétri à l'étuve dans les conditions optimum de germination. Deux méthodes ont été employées, soit le trempage des graines dans les extraits 
pendant 24 ou 48 heures avant l'épreuve, soit l'arrosage des graines avec les extraits pendant l'opération. Ife tableau XX donne les résultats d'ensemble. On remarque que le miel et la propolis sont les plus inhibiteurs de la germination des graines de riz, aucun grain n'ayant germé I5 jours après la mise en expérience. L'extrait d'abeille et le pollen retardent la germination jusqu'au $9^{\mathrm{e}}$ jour (les plantules restent deux fois plus petites que chez le témoin). La cire donne un résultat un peu différent, la germination (60 p. Ioo des grains seulement) a lieu au bout de trois jours, mais la plantule reste minuscule et ne se développe pas. Avec la gelée royale, la germination a lieu au bout de 3 jours également, la plantule se développe ensuite mais reste d'une taille moitié moindre que les témoins. Les témoins ayant germé normalement au bout de 3 jours, je puis rapprocher de ces essais l'expérience par laquelle LEMAX a montré que le venin d'abeille s'est révélé antigerminatif pour les graines. On peut déduire de cet ensemble de résultats que les substances provenant de la ruche, antigerminatives pour le pollen et pour les graines, sont en même temps douées d'une action antibiotique vis-à-vis des souches bactériennes. Dans l'ensemble la propolis et le miel se sont révélés les plus puissants inhibiteurs.

\section{$3^{\circ}$ Valeur antifongique des produits de la ruche.}

Nous avons déjà vu que les extraits d'abeilles, les extraits de pollen récoltés par les abeilles et les extraits de gelée royale n'étaient pas antifongiques. D'autre part Tausson a montré que Aspergillus flavus peut se développer sur de la cire, Moreinini que la gelée royale n'inhibe pas le développement des levures et nous savons que le miel fermente facilement si la teneur en eau est suffisante. Il semble donc a priori que la colonie d'abeilles soit mal défendue sous ce rapport. I,es expériences précédentes sur la germination des pollens et des graines m'ont permis indirectement d'éprouver la valeur antifongique de tous les produits de la ruche. Les résultats sont portés sur le tableau XX. On remarque que seule la propolis a une activité antifongique très importante. Pendant plus d'un mois des plaques de gélose contenant des extraits de propolis et laissées à l'air libre n'ont pas été envahies par les moisissures. I a gelée royale a permis un développement assez faible et sur l'extrait de miel il a fallu iz jours pour obtenir quelques colonies de moisissures. Les extraits d'abeilles, de cire et de pollen en pelotes n'empêchent pas la croissance des champignons et une flore abondante couvre les boîtes de gélose dès le $3^{\mathrm{e}}$ jour. Il se développe des moisissures sur les témoins, mais moins que sur les deux extraits précédents, sans doute parce que les premiers manquent de substances de croissance. J'ai également éprouvé la valeur antifongique de l'extrait antibiotique de pollen en pelotes de l'abeille; les résultats montrent l'inactivité de ces substances vis-à-vis de Pericystis apis. En 
résumé, on peut dire que seule la propolis est intéressante au point de vue antifongique dans la ruche. En effet, les cadavres (souris ou gros lépidoptères) dont les abeilles ne peuvent se débarrasser à cause de leur taille sont enrobés de propolis. Ces cadavres ne se putréfient pas, mais ils ne moisissent pas non plus, bien que dans la ruche il se développe parfois des moisissures sur les rayons, les larves, les abeilles adultes et le pollen, en général dans les zones abandonnées par les abeilles. On peut donc voir là 1'utilité d'un facteur antifongique dans la propolis.

\section{$4^{\circ}$ Action antibiotique de Bacillus larvae.}

Il ne m'a pas semblé possible de passer en revue les produits de la ruche sans signaler que le Bacillus larvae, souche pathogène très spécifique du couvain d'abeilles, possède également des propriétés antibiotiques. Les différentes personnes chargées du diagnostic ou de l'étude des maladies du couvain de l'abeille ont remarqué que presque toujours le Bacillus larvae se trouve en culture pure dans les écailles desséchées au fond des cellules. HoLsT (I945) ayant noté lui aussi que les cultures sont pures dans les cadavres de larves mortes de loque américaine a pensé que Bacillus larvae devait contenir un facteur antibiotique vis-à-vis des autres souches bactériennes et en a montré l'action antibiotique sur une douzaine de souches; parni celles-ci : Escherichia coli, Staphylococcus aureus, Bacillus subtilis, Bacillus alvei. Le principe actif est soluble dans 1'eau, mais insoluble dans les solvants organiques et dans l'alcool. Sa stabilité est modérée à la chaleur, mais la stérilisation par la chaleur est toutefois possible. L'extrait est toxique pour la souris par voie intrapéritonéale et non toxique par voie buccale. L'action inhibitrice est variable suivant les souches; par exemple, l'extrait agit à la concentration de I/200o pour Staphylococcus aureus, et à I/20 ooo pour Escherichia coli. Ces travaux sont restés inachevés à cause de la mort de HoLsT, mais ils mériteraient d'être repris et complétés. Il est bon de remarquer notamment l'action inhibitrice sur Bacillus alvei responsable de la loque européenne. Les observations innombrables faites sur les colonies malades confirment en effet qu'il est excessivement rave qu' une colonie d'abeilles atteinte de loque américaine soit également atteinte par la loque européenne en mêne temps. 
ChAPI'TRE, VII

\section{CONCLUSIONS DE LA DEUXIEUE PARTIE}

J'ai montré que les différents produits de la ruche possédaient des propriétés antibiotiques à des degrés variables. Lors de l'étude de chacun d'eux, j'ai essayé de les comparer aux autres suivant leur origine, leur utilisation pour l'abeille et leur action sur les bactéries. Cependant, ces comparaisons étant fragmentaires, j'ai regroupé ici les rapports qui existent entre tous les produits de la ruche vis-à-vis des souches bactériennes étudiées, et plus spécialement vis-à-vis des souches pathogènes de la colonie d'abeilles.

\section{$I^{0}$ Comparaison de l'action antibiotique des divers produits de la ruche et de l'abeille.}

Si j'examine les courbes des figures 26 et 27 , je puis remarquer tout d'abord qu'il existe deux séries de substances.

a) Un lot très homogène, dont les courbes sont très groupées ; il s'agit de la propolis, de la cive, et du miel.

b) Un lot très disparate plus actif que le précédent comprenant la gelée royale, le pollen et l'abeille. I a gelée royale étant la substance la plus voisine du groupe précédent et le pollen étant la substance dont l'action antibiotique se différencie le plus de toutes les autres.

Cependant, quelques remarques générales sont valables pour l'ensemble des substances éprouvées. Les tableaux XXI et XXII résument l'action de ces substances sur une dizaine de souches bactériennes.

a) L'action antibiotique est faible on nulle pour les Escherichia coli et Pseudomonas pyocyanea.

b) L'activité antibiotique est plus grande sur Bacillus alvei que sur Bacillus larvae.

c) L'action antibiotique est importante sur Salmonella gallinarum (sauf pour le miel et la propolis) et sur Proteus vulgaris.

d) I'activité antibiotique du pollen est, pour toutes les souches, plus importante que les autres substances sauf pour les souches pathogènes de la ruche pour lesquelles l'extrait d'abeille est plus actif. 
e) Les deux extraits qui se ressemblent le plus sont ceux $d u$ miel et de la cire.

f) Les extraits de bourgeons de Populus ont une action très voisine de ceux de propolis. D'autre part, on retrouve dans le miel, la cire et la propolis un colorant, la chrysine qui provient des bourgeons de peuplier. Or, l'activité antibiotique de ces trois produits de l'abeille est voisine. Il est possible qu'il y ait diffusion dans le miel d'un autre corps provenant de la propolis ou de la cire (contenant elle-même de la propolis), puisque l'antibiotique de la propolis est extractible par l'eau.

\section{TABLEAU XXI}

Comparaison de l'activité antibiotique des diverses substances de la ruche.

\begin{tabular}{|c|c|c|c|c|c|c|}
\hline Souches éprouvées & Extrait abeille & $\begin{array}{c}\text { Extrait } \\
\text { miel }\end{array}$ & $\begin{array}{c}\text { Extrait } \\
\text { cire }\end{array}$ & $\begin{array}{l}\text { Extrait } \\
\text { propolis }\end{array}$ & $\begin{array}{c}\text { Extrait } \\
\text { gelée royale }\end{array}$ & $\begin{array}{c}\text { Extrait } \\
\text { pollen stocké }\end{array}$ \\
\hline S. pullorum 309 & \multirow{2}{*}{+ tous les extraits } & \multirow{2}{*}{+} & +- & \multirow{3}{*}{+-} & +- & \multirow{10}{*}{+} \\
\hline S. gallinarum $n^{0} 3^{8}$ & & & + & & + & \\
\hline S. Dublin $n^{0} \quad 7-54$ & \multirow{5}{*}{$\mid \begin{array}{l}\text { avec abeilles de } \\
3 \text { à I } 2 \text { jours } \\
\\
\text { - avec abeilles de } \\
\text { moins de - de } 3 \\
\text { jours et de }+ \text { de } \\
\text { I2 jours. }\end{array}$} & +- & +- & & +- & \\
\hline $\begin{array}{l}\text { E. Coli o III }=B_{4} \text {. } \\
\text { EC } 55 \text { I }\end{array}$ & & \multirow{3}{*}{-} & \multirow{3}{*}{-} & \multirow{4}{*}{-} & - & \\
\hline $\begin{array}{l}\text { E. Coli o } 55=B_{5} \text {. } \\
\text { Ec } 5401\end{array}$ & & & & & $+\cdots$ & \\
\hline $\begin{array}{l}\text { E. Coli } \circ 26=\text { B6. } \\
\text { Ec } 5434\end{array}$ & & & & & - & \\
\hline E. Coli Bordet & & +- & +- & & & \\
\hline Proteus vulgaris & + (tous les extraits) & + & + & + & + & \\
\hline $\begin{array}{l}\text { Pseudomonas pyo- } \\
\text { cyanea } 4\end{array}$ & $\begin{array}{l}+ \text { avec abeilles de } \\
\text { r } 2 \text { jours. } \\
\text { - avec tous les au- } \\
\text { tres extraits. }\end{array}$ & +- & - & - & $\cdots$ & \\
\hline $\begin{array}{l}\text { B. subtilis souche } \\
\text { Caron }\end{array}$ & $+($ tous les extraits $)$ & + & + & + & + & \\
\hline Otsservations & \begin{tabular}{|} 
Jamais actifs: \\
Abeilles naissantes \\
et très vieilles \\
abrilles
\end{tabular} & & & & & $\begin{array}{l}\text { L'extrait de de } \\
\text { pollen est très } \\
\text { actif sur } 5 . \\
\text { gallinarum et } \\
\text { Proteus vul- } \\
\text { garis. }\end{array}$ \\
\hline
\end{tabular}

g) Aucun rapport dans l'action antibiotique n'est visible entre le pollen et la gelée royale et, contrairement à toute attente, la gelée royale qui contient certains principes du pollen plus concentrés (acide pantothé- 
nique par exemple) est moins active que celui-ci. Au contraire, le vieillissement des deux substances qui n'existe pas pour les autres produits de la ruche est très marqué pour la gelée royale et le pollen. Ce vieillissement qui n'affecte pas la valeur antibiotique des extraits de pollen annule rapidement cette valeur dans les extraits de gelée royale.

h) Les antibiotiques de la cire et de la propolis sont très stables, celui du miel est photolabile, mais il est thermostable.

\section{TABLEAU XXII}

Comparaison de l'activité antibiotique entre diverses substances de la ruche.

\begin{tabular}{|c|c|c|c|c|c|c|}
\hline Souches éprouvées & $\left|\begin{array}{l}\text { Extrait } \\
\text { abeille }\end{array}\right|$ & $\begin{array}{c}\text { Extrait } \\
\text { miel }\end{array}$ & $\begin{array}{c}\text { Extrait } \\
\text { cire }\end{array}$ & $\begin{array}{l}\text { Extrait } \\
\text { propolis }\end{array}$ & $\begin{array}{l}\text { Extrait } \\
\text { pollen } \\
\text { stocké en } \\
\text { rayons }\end{array}$ & $\begin{array}{c}\text { Extrait } \\
\text { gelće } \\
\text { royale }\end{array}$ \\
\hline $28 \mathrm{~B}$. Subtilis souche Caron ... & + & + & + & + & + & + \\
\hline 26 Pseudomonas pyocyanea.... & + & +- & - & - & + & - \\
\hline 23 Proteus vulgaris ... & +++ & + & + & + & $+t+t$ & $+t$ \\
\hline 20 E. Coli Bordet ......... & + & +- & +- & - & ++ & - \\
\hline I 6 E. Coli o $26=$ B6. Fe 5434 & + & 一 & -- & - & + & - \\
\hline I3 E. Coli ○ $55=$ B $_{5}$. Ec $540 \mathrm{I}$ & + & - & - & 一 & + & +- \\
\hline Iо E. Coli o I I I $=B_{4}$. Ec 5512 & +- & - & - & - & + & - \\
\hline 9 S. type Dublin No $7-54 \ldots$. & + & + & +- & +- & ++ & +- \\
\hline + S. gallinarum $n^{\circ} 3^{8} \ldots \ldots$ & ++ & + & + & +- & ++++ & + \\
\hline 3 S. pullorum no $309 \ldots \ldots \ldots$ & ++ & +- & + & +- & +++ & $+\longrightarrow$ \\
\hline 29 B. alvei...... & +++ & + & + & + & $+t$ & + \\
\hline 30 B. larvae. . & + & +- & +- & +- & + & +- \\
\hline
\end{tabular}

i) Enfin, aucun des extraits, même celui de la gelée royale, n'a de ressemblance vraiment nette avec l'extrait d'Apis mellifica. L'antibiotique de l'abeille est différent des autres produits de la ruche et cependant, à part la propolis qui est d'origine végétale pour la plus grande part, on peut dire que l'abeille ajoute de ses propres sécrétions salivaires à tous ces produits nectar, pollen et cire (malaxage de cette substance lors de la construction). Quant à la gelée royale, c'est une sécrétion des glandes pharyngiennes et le rapport avec l'antibiotique de l'abeille devrait être plus net à priori. I,e tableau XXIII essaie de donner une image des rapports existant entre les différentes substances et l'abeille, mais, s'il permet de rendre compte de certaines similitudes (cire, miel et propolis) 
il marque bien certaines différences d'activité antibiotique (pollen et gelée royale ou gelée royale et abeille).

\section{TABLEAU XXIII}

Schéma montrant les rapports existant entre les différents produits de la ruche et l'abeille.

\begin{tabular}{|c|c|c|c|c|c|}
\hline Substance & Code & Origine principale & $\begin{array}{l}\text { Addition par } \\
\text { l'abeille ou } \\
\text { diffusion dans } \\
\text { la ruche }\end{array}$ & & Formule finale \\
\hline Abeille & 0 & $\begin{array}{c}\text { endogène } \\
\text { (glandespharyngiennes) }\end{array}$ & & 0 & \multirow{2}{*}{$\begin{array}{l}\text { Ces extraits sont } \\
\text { très différents au } \\
\text { point de vue } \\
\text { antibiotique }\end{array}$} \\
\hline Gelée royale & 0 & endogène & & 0 & \\
\hline $\begin{array}{c}\text { Pollen } \\
\text { (Récolté par } \\
\text { l'abeille) }\end{array}$ & $\ominus$ & exogène & +0 & $\ominus$ & +0 \\
\hline Miel & + & exogène & $O \Delta \square$ & + & \multirow{3}{*}{$\begin{array}{ll}\triangle \square & \begin{array}{c}\text { Ces extraits } \\
\text { sont voisins au }\end{array} \\
\square \bigcirc \quad \begin{array}{l}\text { point de vue } \\
\text { antibiotique }\end{array} \\
\triangle & \text { a }\end{array}$} \\
\hline Cire & $\triangle$ & $\begin{array}{c}\text { endogène } \\
\text { (glandes cirières) }\end{array}$ & $\square 0$ & $\triangle$ & \\
\hline Propolis & $\square$ & exogène (Populus) & $\square \triangle$ & $\square$ & \\
\hline
\end{tabular}

\section{$2^{\circ}$ Valeur antibiotique des divers produits de la ruche et de l'abeille vis-à-vis de Bacillus alvei et de Bacillus larvae.}

L'activité antibactérienne des produits de la ruche vis-à-vis des souches pathogènes de la colonie d'abeilles a été très peu étudiée, et seuls quelques travaux fragmentaires déjà cités plus haut ont été effectués, à savoir, l'action sur $B$. larvae ou $B$. alvei des nourritures larvaires (Toumanoff I929, Tarr I934, Matuka et Topolnik I952, Krasikova I955) de la propolis (VERGÉ, I95I) et du pollen (travaux plus complets que les précédents par SMith, Beck et ANderson (I949).

J'ai entrepris d'éprouver tous les extraits de produits de la ruche et de l'abeille sur Bacillus larvae et Bacillus alvei. Les résultats ont déjà été portés à chaque chapitre concernant l'une ou l'autre des substances. Dans ces conclusions, je veux simplement grouper l'ensemble de ces résultats sous forme de tableaux et en tirer des règles générales concernant 1'activité antibiotique de la ruche vis-à-vis des souches pathogènes pour l'abeille.

J'ai éprouvé successivement par la méthode de la dilution :

$\mathrm{I}^{0}$ L'extrait alcoolique d'abeilles saines.

$2^{\circ}$ L'extrait alcoolique d'abeilles dont la colonie était atteinte de Loque américaine (sur $B$. larvae seulement). 
$3^{\circ}$ L'extrait alcoolique de pollen en pelotes récolté par les abeilles.

$4^{0}$ L'extrait alcoolique de cire bâtie (très foncée).

$5^{\circ}$ L'extrait alcoolique de propolis.

$6^{\circ}$ L'extrait acétonique de miel de colza.

$7^{\circ}$ L'extrait éthéré de gelée royale.

Suivant la technique habituelle, les extraits en phase aqueuse furent ajoutés aux milieux de culture employés pour les témoins. Pour Bacillus

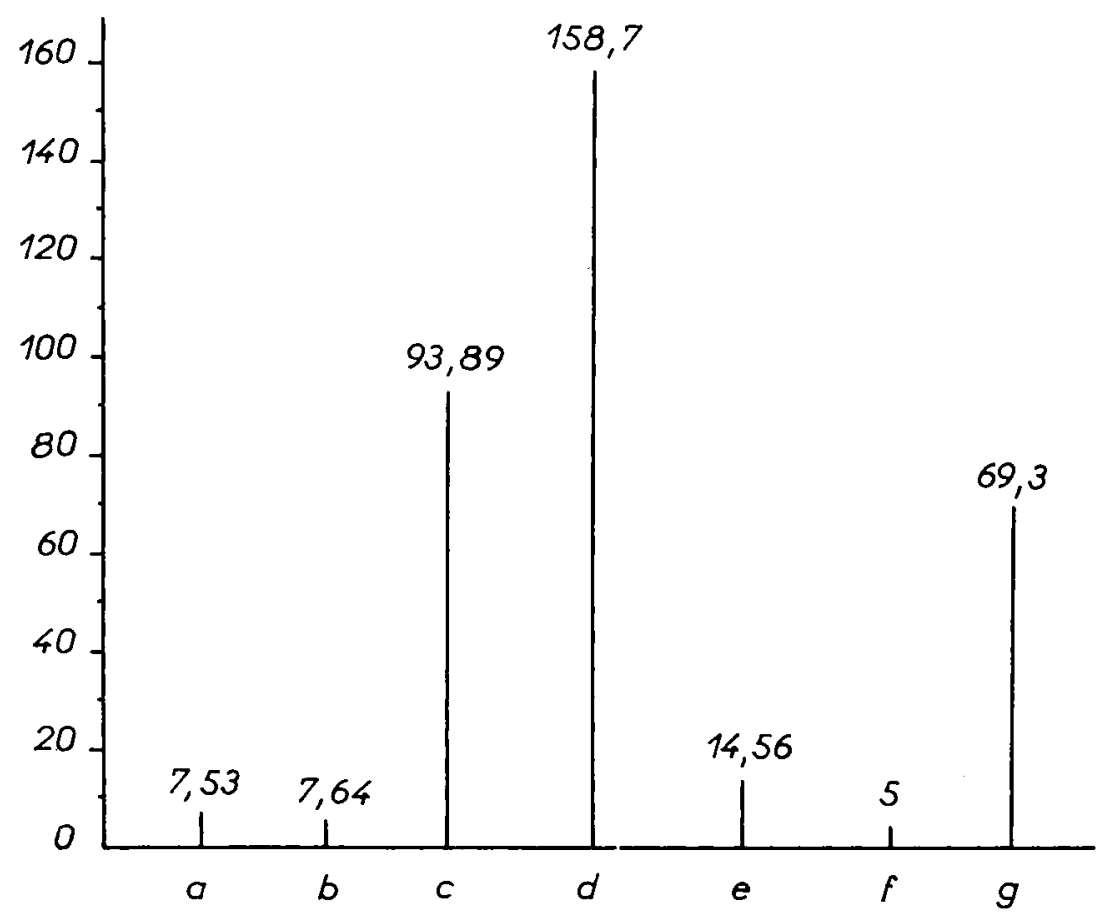

Fig. $3^{6}$. - Action de quelques substances antibiotiques de la ruche sur Bacillus alvei.

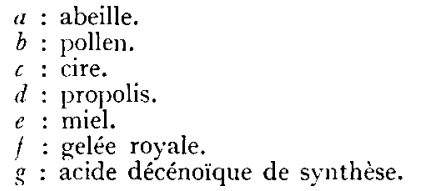

alvei, le milieu de culture est un milieu à Io p. I ooo de peptone, gélosé à 25 p. I ooo ; Bacillus larvae, plus difficile à cultiver, a été ensemencé, sur milieu de Dubos au " tween 80 ".

Si j'interprète les résultats des figures 36 et 37 , et des table aux XXIV et XXV, je constate les faits suivants :

I $^{\text {o }}$ 'action antibiotique des extraits d'abeilles ou de divers produits de la ruche est sensiblement parallèle pour Bacillus larvae ou Bacillus alvei : action très importante des extraits de propolis et de cire sur ces deux bacilles, tandis que les autres extraits sont beaucoup moins actifs. 
$2^{\circ}$ L'action antibiotique des divers extraits est plus marquée envers Bacillus alvei qu'envers Bacillus larvae dans tous les cas, et très souvent double ou triple.

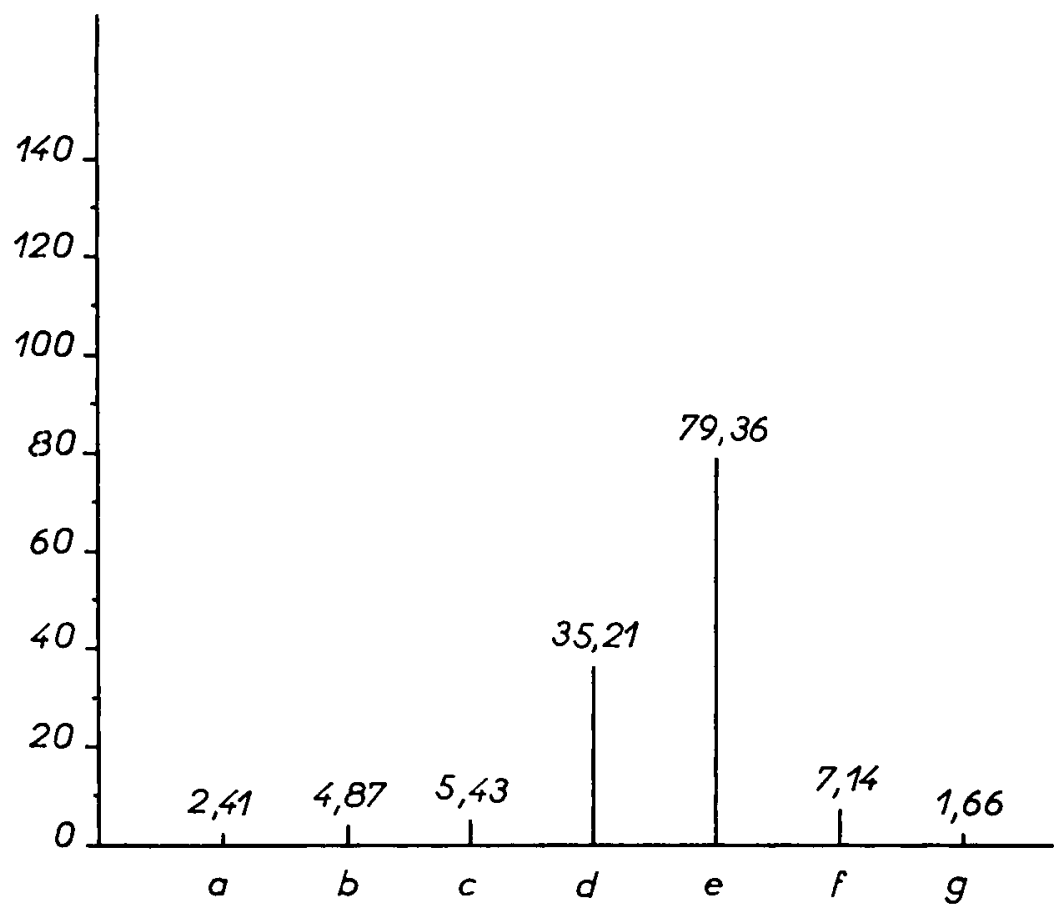

Fig. 37. - Action de quelques substances antibiotiques de la ruche sur Bacillus lariae. $a$ : abeilles saines.

$b$ : abeilles dont le couvain est atteint de la loque américaine.

$c$ : pollen.

$d:$ cire.

$e$ : propolis.

$f$ : miel.

$g$ : gelée royale.

\section{TABLEAU XXIV}

Action de quelques substances antibiotiques de la ruche sur Bacillus alvei.

\begin{tabular}{|c|c|c|c|}
\hline Matériel utilisé & Mode d'extraction & $\mathrm{U}$ & Observations \\
\hline 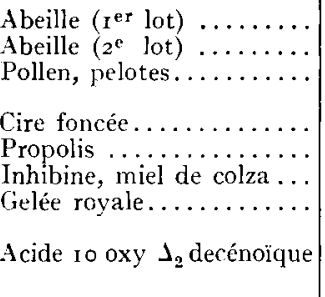 & $\begin{array}{l}\text { Alcool } \\
\text { Alcool } \\
\text { Alcool } \\
\text { Acétonte } \\
\text { Alcool } \\
\text { Acétone } \\
\text { Éther }\end{array}$ & \begin{tabular}{|c|}
7,53 \\
7,24 \\
I 0,86 \\
93,89 \\
I 58,7 \\
I 4,56 \\
Action avecl'extrait de \\
Ig G. R. fraiche \\
Action avec I 4,4 \\
mg d'acide.
\end{tabular} & $\begin{array}{l}\qquad- \\
\text { Retard juste avec } \\
\text { la dilution limite. } \\
- \\
- \\
-\end{array}$ \\
\hline
\end{tabular}


$3^{\circ}$ L'action des divers extraits sur Bacillus alvei consiste en une inhibition à partir d'une certaine concentration et un développement normal au-dessous de cette concentration. Seul l'extrait de pollen possède en plus un effet retardateur sur le développement de la souche lorsqu'il est ajouté au milieu même en quantité inférieure à la dilution limite nécessaire à l'inhibition.

\section{TABLEAU XXV}

Actions de quelques substances antibiotiques de la ruche sur Bacillus larvae.

\begin{tabular}{|c|c|c|}
\hline Matériel utilisé & I & Observations \\
\hline $\begin{array}{l}\text { Abeille saine } \\
\qquad \text { (Extrait alcool) } \ldots\end{array}$ & $2,4 \mathrm{I} \quad(\mathrm{I}, 5 \mathrm{mII})$ & $\begin{array}{l}\text { Même extrait que celui éprouvé sur B. alvei ( } 2^{\mathrm{e}} \text { lot). } \\
\text { Le témoin se développe en } \mathrm{z} \text { h. Trouble en } 48 \mathrm{~h} \\
\text { en dessous de la limite ( } \mathrm{ml} \text { ). }\end{array}$ \\
\hline $\begin{array}{l}\text { Abeille loque américaine } \\
\text { (Extrait alcool) } \ldots . . .\end{array}$ & $4,87(4 \mathrm{ml})$ & $\begin{array}{l}\text { A } 3 \mathrm{ml} \text { développement en } 36 \mathrm{~h} \text {. } \\
\text { A } 2 \mathrm{ml} \text { développement en } 24 \mathrm{~h} \text {. } \\
\text { témoin en I } 2 \mathrm{~h} \text {. }\end{array}$ \\
\hline $\begin{array}{l}\text { Pollen en pelote } \\
\text { (Extrait alcool) }\end{array}$ & $5,43(2 \mathrm{ml})$ & $\begin{array}{l}\text { Léger retard } \\
\text { Anneau bactérien } 36 \mathrm{~h} \text { avec } \mathrm{I}, 5 \mathrm{ml} \\
\text { Anneau bactérien } 24 \mathrm{~h} \text { avec } \mathrm{I} \mathrm{ml} \\
\text { Anneau bactérien } \mathrm{I} 2 \mathrm{~h} \text { avec témoin }\end{array}$ \\
\hline $\begin{array}{l}\text { Cire foncée } \\
\text { (Iixtrait acétone) }\end{array}$ & $35,2 \mathrm{I}(2 \mathrm{ml})$ & $\begin{array}{l}\text { Développement } 36 \mathrm{~h} \text { avec } \mathrm{I}, 5 \mathrm{ml} \\
\text { Développement } 24 \mathrm{~h} \text { avec } \mathrm{I} \mathrm{ml} \\
\text { Développement i } 2 \mathrm{~h} \text { avec témoin }\end{array}$ \\
\hline $\begin{array}{l}\text { Propolis } \\
\text { (Extrait alcool). }\end{array}$ & $79,36(2 \mathrm{ml})$ & $\begin{array}{l}\text { Développement } 36 \mathrm{~h} \text { avec } \mathrm{I}, 5 \mathrm{ml} \\
\text { Développement } 24 \mathrm{~h} \text { avec } \mathrm{i} \mathrm{ml} \\
\text { Développement } \mathrm{i} 2 \mathrm{~h} \text { avec témoin }\end{array}$ \\
\hline $\begin{array}{l}\text { Inhibine de miel de colza } \\
\text { (Lxtrait acétone) } \ldots .\end{array}$ & $7, \mathrm{I} 4$ & $\begin{array}{c}\text { Développement } 36 \mathrm{~h} \text { avec } 100 \mathrm{mg} \text { d'extrait sec } \\
\text { Développement } 12 \mathrm{~h} \text { avec moins de roo } \mathrm{mg} \\
\text { d'extrait sec }\end{array}$ \\
\hline $\begin{array}{l}\text { Gelée royale } \\
\text { (Extrait éthéré) }\end{array}$ & $\begin{array}{l}\text { Action a vec l'extrait de } \\
3 \mathrm{~g} \text { de G. R. fraîche }\end{array}$ & $\begin{array}{c}\text { Avec l'extrait de } 2 \mathrm{~g} \text { de } \mathrm{G} . \mathrm{R} \text {. retard de } 48 \mathrm{~h} \\
\text { dans le développentent }\end{array}$ \\
\hline
\end{tabular}

$4^{\circ}$ Au contraire, 1'action des divers extraits sur Bacillus larvae est différente. En effet, tous les extraits possèdent une action retardatrice sur le développement du bacille, lorsque ceux-ci sont employés en dessous de la dilution inhibitrice.

$5^{\circ} \mathrm{L}_{1}$ 'extrait d'abeilles provenant de colonies atteintes de la I,oque américaine contient deux fois plus d'unités antibiotiques sur Bacillus larvae que l'extrait d'abeilles saines, comme si une sécrétion antibiotique "de défense" se produisait à ce moment.

$6^{\circ}$ L'action de la gelée royale est très faible sur Bacillus alvei et encore plus faible sur Bacillus larvae. Pour Bacillus larvae, il a fallu 30 p. Ioo de gelée royale dans un milieu pour obtenir l'inhibition totale (pH ajusté) et pour Bacillus alvei ro p. Ioo seulement. 
- En conclusion, on peut dire que l'intérieur de la ruche parait entièrement tapissé de substances antibiotiques qui montrent une certaine activité vis-à-vis des maladies bactériennes du couvain. Les provisions (pollen et miel), et même dans une plus faible mesure la gelée royale, participent à cette défense chimique contre les infections. Cependant l'importance réelle de cette défense reste à déterminer de manière plus précise.

\section{$3^{0}$ Signification des facteurs antibiotiques présents dans la colonie d'abeilles.}

L'origine des substances présentes dans la ruche est, nous l'avons vu, très variée. Certains corps sont récoltés sur les végétaux et entreposés directement, d'autres sont modifiés avant d'être emmagasinés, d'autres enfin sont des sécrétions de l'insecte lui-même. Certaines substances sont utilisées à édifier le nid, d'autres servent à la nourriture. A travers cette diversité nous n'avons pas réellement trouvé de rapports très nets du point de vue de la valeur antibiotique, comme je 1'ai montré ci-dessus. Les substances diverses de la ruche ont des effets antibactériens parfois complémentaires et suivant les besoins, il doit se produire un équilibre. Ces besoins peuvent être saisonniers et suivre le développement du couvain; ils ont peut-être aussi un rapport avec les maladies et avec le stockage des produits récoltés. L' "antibiotique " présent partout dans la ruche permet à celle-ci de conserver ses denrées, de lutter contre les maladies et d'embaumer avec succès les gros cadavres. Par exemple, la cire des rayons paraît revêtue tout entière d'une " pellicule " antibiotique, formée de la propolis dont les abeilles la vernissent. Une certaine asepsie est maintenue dans la colonie non seulement grâce aux matériaux de construction (cire et propolis), mais encore grâce aux provisions ellesmêmes (miel et pollen) et, dans une plus faible mesure, grâce à la gelée royale constamment dégorgée dans les cellules du couvain.

Je ne puis m'empêcher de comparer cette défense chimique contre les infections à celle que l'on rencontre d̀ l'intérieur d'un organisme vivant. 


$$
\text { . }
$$




\section{TROISIÈME, PAR'TIE}

\section{LES SUBSTANCES ANTIBIOTIQUES CHEZ DIVERS INSECTES.}

\section{Généralités.}

Nous savons que de nombreux insectes possèdent des substances antibactériennes, antifongiques et même insecticides vis-à-vis des autres espèces. Ces notions ne sont solidement établies que depuis quelques années grâce aux recherches de PAvan et de Koinsumi dont nous avons analysé les principaux travaux. Après avoir obtenu des résultats positifs sur l'abeille, j'ai éprouvé également la valeur antibactérienne de quelques insectes afin de savoir si la présence d'antibiotique était un phénomène général ou réservé seulement aux insectes sociaux et à certains groupes d'animaux privilégiés. Les premiers essais ont consisté à plonger les animaux entiers, sans précautions spéciales d'asepsie, dans des milieux de culture synthétiques appropriés et stérilisés. Les milieux sont examinés ensuite I2 heures ou 24 heures après cet ensemencement pour voir s'il existe un développement microbien. Les premières épreuves ont porté sur Musca domestica, Calliphora vomitoria, Chrysopa perla, Drosophila melanogaster et Forficula auricularia. J'ai obtenu un développement bactérien intense dans tous les cas. Des répétitions nombreuses ont donné les mêmes résultats. Ces premières expériences ont été reprises d'une manière plus complète, comme nous le verrons plus loin. Mais avant d'exposer mes recherches personnelles, il est utile, je pense, de montrer que le sujet n'est pas neuf et que de nombreuses observations et travaux existent là-dessus dans la littérature. Cependant, je passe rapidement sur toutes les observations sujettes à caution concernant notamment l'utilisation d'insectes comme médicaments, que nous pouvons trouver dans 1'ancienne pharmacopée.

Tout d'abord le célèbre médecin Ambroisf Paré avait remarqué vers 1579 l'effet bénéfique de certaines larves de diptères pour la guérison des plaies. Le chirurgien LARREY (I803) médecin des armées napoléoniennes avait précisé l'action des larves de diptères myasogènes sur les plaies lors des campagnes d'Egypte et de Syrie. Des essais systématiques sur 1'homme furent réalisés pendant la guerre I9I4-I9I8, par BAER (I93I). Ces traitements furent réalisés au moyen de larves de Phaenicia sericata 
(Meigen) et également de Phormia regina (Meig.) et de Lucilia caesar (I..). $L_{1}$ es résultats obtenus déclenchent alors une foule de travaux sur ce sujet. Mais il faut attendre 1934 pour que MAURice réussisse à montrer qu'un extrait aqueux de larve de $P h$. sericata est aussi actif que la présence des larves elles-mêmes. Enfin, Gwatkin et Fallis (I938) montrent que l'eau de lavage des larves de Calliphora possède des propriétés antibactériennes. Bösiger (renseignement oral, non publié, 1957) m'a signalé qu'il avait remarqué lors de travaux sur Drosophila melanogaster que les larves possédaient des propriétés antibiotiques. Nous avons déjà vu l'action antibactérienne des extraits de larves de Melasoma populi $\mathrm{I}_{1}$. (PAvan, I953) due à l'aldéhyde salicylique ainsi que l'action bactéricide du sang d'Agriotis pronubana (mis en évidence par PAILLOT) et du sang d'insectes divers (GLASER I9I8), mais sans que cette action soit constante. Plus proches de nous et plus importants, sont les travaux de Pavan sur divers insectes et principalement sur Iridomyrmex pruinosus humilis, que j'ai analysés dans la première partie de cette thèse. De même, des substances antibiotiques ont été signalées chez les fourmis par RAIGNIER (I947) et STTMPPER (I950). RAIGNIER signale des faits se rapportant à la valeur fongostatique des nids de Formica rufa et pense que le $\mathrm{pH}$ joue un grand rôle dans les fourmilières. STUmper, de son côté, trouve que le carton des nids (non abandonnés) de Lasius fuliginosus contient des substances antibiotiques. Cependant, il semble que ces facteurs seraient plutôt antifongiques, puisqu'ils inhibent le développement des moisissures. D'autre part, PAVAN avait déjà trouvé que Lasius recèle une substance antibiotique principalement dans la tête et l'abdomen. Je pense que ces quelques exemples suffisent à montrer la présence de ces facteurs antibiotiques ou antifongiques chez les insectes, aussi bien chez les larves, que chez les adultes et aussi bien chez ies insectes sociaux que les autres sans distinction de famille ou de groupe.

Je reviendrai maintenant à quelques notions générales concernant l'infection chez les insectes.

D'après PaILLOT, les insectes adultes sont moins atteints par les épizooties que les larves ou les chenilles. Il en est de même chez les abeilles. Peut-être l'histolyse des tissus lors des métamorphoses facilite-t-elle le passage des bactéries ou des champignons dans le sang. Chez l'insecte adulte, les blessures externes du tégument favorisent également l'infection en laissant pénétrer dans la cavité générale les éléments microbiens (VAGO, 1959). D'après de nombreux auteurs, les insectes s'infectent davantage par le tégument que par le tube digestif. Les maladies bactériennes des insectes sont plus souvent des septicémies que d'autres affections. J'ai vu chez l'abeille que l'âge de l'insecte adulte avait une grande importance pour la production del'antibiotique; il existerait une véritable maturation du tégument. Bourn et VELU ont montré également l'influence de l'âge 
des insectes sur les infections microbiennes. I,eurs travaux ont été réalisés sur Schistocerca peregrina avec Coccobacillus acridiorum. Enfin la connaissance approfondie des constituants de la cuticule des insectes, dont $j$ 'ai déjà exposé dans la première partie ce qui nous intéresse ici, aide à la compréhension des mécanismes de défense biochimique vis-à-vis des microbes pathogènes. C'est la présence d'acides gras libres dans la cuticule qui permet, dans une certaine mesure, par leur valeur antifongique ou antibiotique, la lutte contre les infections microbiennes. Nous avons vu que l'abeille blessée, même légèrement, meurt très rapidement. On peut supposer que sa cuticule lui sert de manteau protecteur contre les invasions bactériennes. Les insectes très sensibles aux mutilations (comme certains lépidoptères ou coléoptères) sont peut-être comme Apis mellifica et possèdent à la surface de leur corps une substance antibiotique protectrice. Au contaire les frelons et les guêpes hyménoptères, peu sensibles aux blessures et aux mutilations utilisent sans doute un moyen de défense différent. 
ChapITRE PREMIER

\section{LES ANTIBIOTIQUES CHEZ LES INSECTES SOCIAUX}

\section{I $^{0}$ Les antibiotiques chez les Trigones et les Mélipones.}

Grâce à 1'obligeance de Virgilio de Portugal. Araujo qui avait expédié au Laboratoire une colonie de Trigona bocandei vivante pour étudier son acclimatation en Europe, j'ai pu éprouver la valeur antibiotique de ces insectes, ainsi que celle de leurs produits.

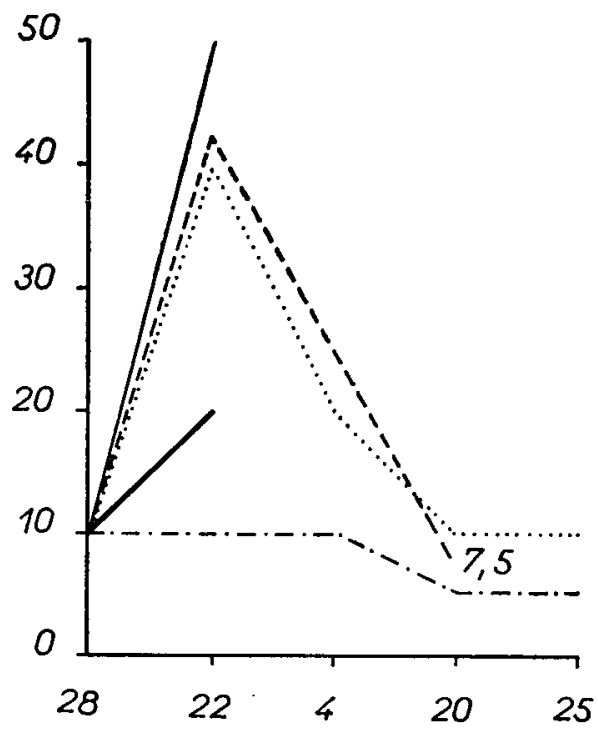

FIG. 38. - Comparaison de l'action antibiotique de l'extrait d'abeille et ceux de quelques insectes (en abscisse les numéros des souches éprouvées).

- Formica rufa.

-.. Vespa crabro.

...... Apis mellifica.

-.... Extrait de Bombus, Arctia caja, coléoptères.

-.... Meliponula bocandei.

Avec 1'extrait alcoolique à chaud et l'insecte lui-même, nous avons obtenu sur Bacillus subtilis, une action deux fois moindre qu'avec l'extrait d'abeille. J'ai essayé l'antibiotique de trigone sur cinq souches bactériennes. La figure 38 montre les résultats obtenus. Si l'on prend la valeur Io comme unité arbitraire pour Bacillus subtilis, on constate que l'activité est identique pour Proteus vulgaris et Salmonella gallinarum, tandis qu'elle 
est moitié moindre sur Escherichia coli Bordet et Pseudomonas pyocyanea. Ces résultats ne montrent pas de concordance avec ceux obtenus chez Apis mellifica, les extraits alcooliques d'abeilles étant très actifs sur les Proteus et les Salmonella.

J'ai éprouvé également des extraits de cire, de propolis et de pollen stocké. J'ai pratiqué l'extraction par l'acétone bouillant (pendant I heure à reflux) de la cire de trigones. Cette cire foncée provenait des " pots " ou grosses cellules à miel et à pollen et je 1'ai soigneusement récoltée et

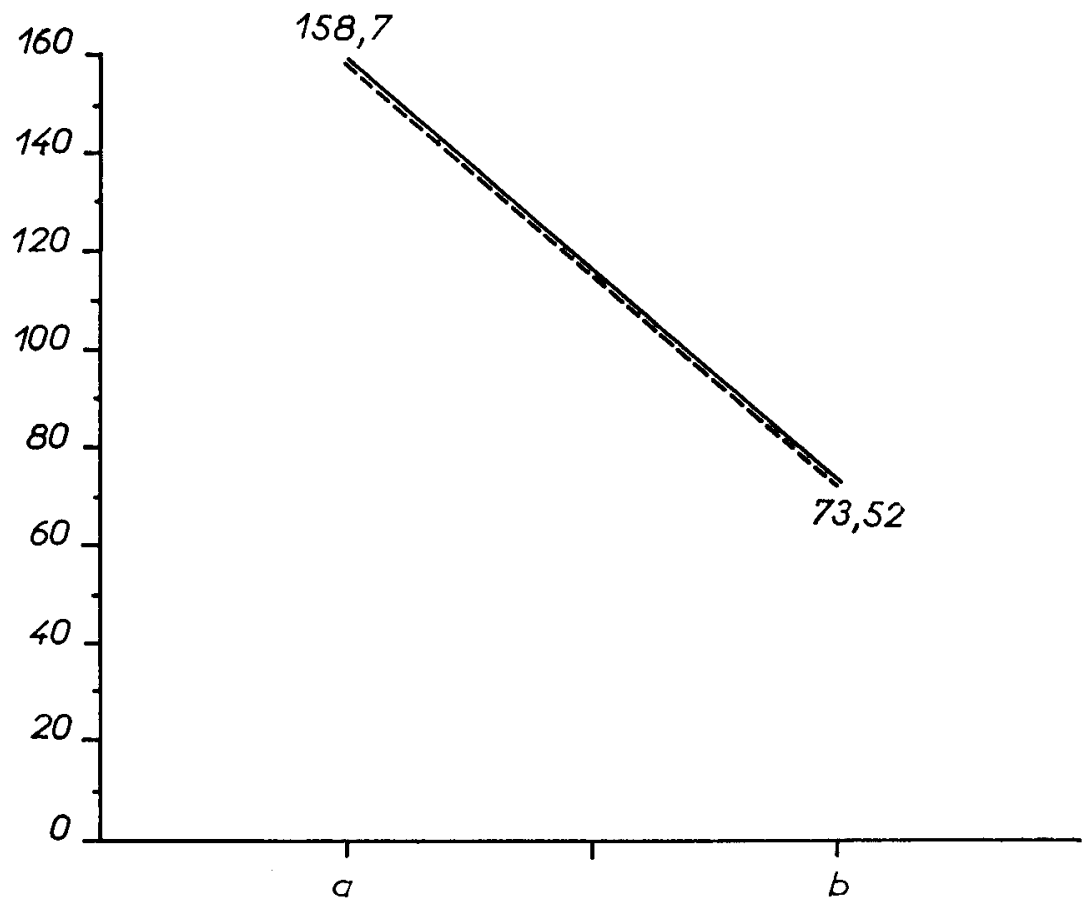

FIG. 39. - Comparaison de l'action antibiotique entre la propolis d'abeille et la résine récoltée par Meliponula bocandei.

- épreuve Proteus vulgaris.

-. épreuve $B$ Subtilis.

$a$ : Propolis récoltée par l'abeille.

$b$ : " résine " récoltée par les mélipones.

lavée auparavant. L'extrait repris par l'eau est acide, comme celui de l'extrait de rayons de l'abeille et son odeur, très voisine. Cet extrait thermostable comme celui de la cire d'Apis a été essayé seulement sur Bacillus subtilis à cause de la petite quantité de matière première en ma possession, mais il est très peu actif ; i’ai trouvé que l'unité Subtilis était voisine de I4 tandis que l'extrait acétonique de la cire d'abeille est Io fois plus actif en moyenne. I1 ne s'agit malheureusement que d'un essai isolé tout à fait insuffisant.

J'ai cherché également quelle pouvait être la valeur antibiotique 
de la " propolis " récoltée par les trigones. La colonie que nous possédions à Bures-sur-Yvette, récoltait d'énormes quantités de résine noirâtre pour calfeutrer les moindres interstices.

Cette " propolis " ou " cérumen ) d'ailleurs voisine de la résine de pin (odeur, consistance) provenait sans doute de la pinède située à une centaine de mètres. J'ai prélevé de cette propolis et également de la propolis dans les colonies d'A pis mellifica voisines. L'extraction alcoolique des deux matières m'a donné des extraits qui, repris en milieu aqueux, ont été essayés sur deux souches bactériennes, Bacillus subtilis et Proteus oulgaris. La figure 39 nous donne le résultat de 1'expérience. Nous pouvons constater que l'activité antibiotique de la propolis d'abeille est plus importante (plus du double) que celle de la résine de Trigona bocandei. D'autre part, on remarque que cette résine a la même action sur Bacillus subtilis que sur Proteus vulgaris que la propolis récoltée par Apis mellifica.

\section{TABLEAU XXVI}

Comparaison entre l'abeille et les autres insectes.

\begin{tabular}{|c|c|c|c|c|c|c|}
\hline Souches essayées & Abeille & $\begin{array}{c}\text { Bombus } \\
\text { terrestris et } \\
\text { lapidarius }\end{array}$ & $\left|\begin{array}{l}\text { Formi- } \\
\text { ca rufa }\end{array}\right|$ & $\left|\begin{array}{c}\text { Reticuliter- } \\
\text { mes lucifu- } \\
\text { gus }\end{array}\right|$ & $\begin{array}{c}\text { Blatella } \\
\text { germanica }\end{array}$ & $\begin{array}{l}\text { Trigona } \\
\text { bocandei }\end{array}$ \\
\hline $28 \mathrm{~B}$. subtilis souche Caron...... & + & + & + & + & - & + \\
\hline 26 Pseudomonas pyocyanea $4 \ldots$ & + & 一 & + & + & 一 & +- \\
\hline 20 E. coli Bordet............. & + & - & + & + & - & +- \\
\hline${ }_{3} 3 \mathrm{E}$ coli $\mathrm{O}_{55}=\mathrm{B}_{5}$. Ec $540 \mathrm{I}$ & + & - & 一 & - & 一 & \multirow{4}{*}{$\begin{array}{c}\text { souches } \\
\text { non } \\
\text { essayées }\end{array}$} \\
\hline IO E. coli $O_{\text {III }}=\mathrm{B}_{4}$. Ec 5512. & +- & - & - & - & - & \\
\hline 16 E. coli $\mathrm{O}_{26} 6=\mathrm{B} 6 . \mathrm{Ec} 5434 \ldots$ & + & - & - & - & - & \\
\hline 9 S. type Dublin $n^{\circ} 7-54 \ldots \ldots \ldots$ & + & - & - & - & $\cdots$ & \\
\hline 4 S. gallinarum $n^{0} 3^{8} \ldots \ldots \ldots$ & ++ & - & + & + & +- & + \\
\hline 3 S. pullorum n $n^{0} 309 \ldots \ldots \ldots$ & ++ & + & + & + & +- & non essayée \\
\hline Proteus vulgaris $\ldots \ldots \ldots \ldots \ldots \ldots$ & +++1 & ++ & +++ & non essayée & non essayée & + \\
\hline
\end{tabular}

L'étude de la valeur antibiotique du pollen stocké par Trigona bocandei a également été entreprise et j'ai tenté de comparer sa valeur avec le pollen stocké par Apis mellifica. I a figure 40 montre l'action des deux extraits sur Bacillus subtilis et Proteus vulgaris. Il faut remarquer que, dans l'un ou l'autre cas, l'activité sur Proteus vulgaris est plus grande: cette bactérie est en effet très sensible à l'action des substances antibiotiques extraites du pollen. Il est impossible de dire si la différence entre ces deux substances est due à la valeur antibiotique du pollen lui-même, 
à la valeur antibiotique des sécrétions ajoutées par ces apides, ou encore, à l'action de fermentations diverses dans les cellules à pollen. I,e pollen stocké par l'abeille était du pollen de Castanea vulgaris presque pur, tandis que le pollen de la colonie de Trigona a révélé à l'analyse pollinique la présence exclusive de pollen exotique (Angola). D'autre part, le pollen de Melipomula stocké dans de grosses cellules semblait à un degré de fermentation supérieur à celui de $1^{\prime} A$ pis mellifica. Ia présence de mycélium indiquait également la possibilité de transformation par des champi-

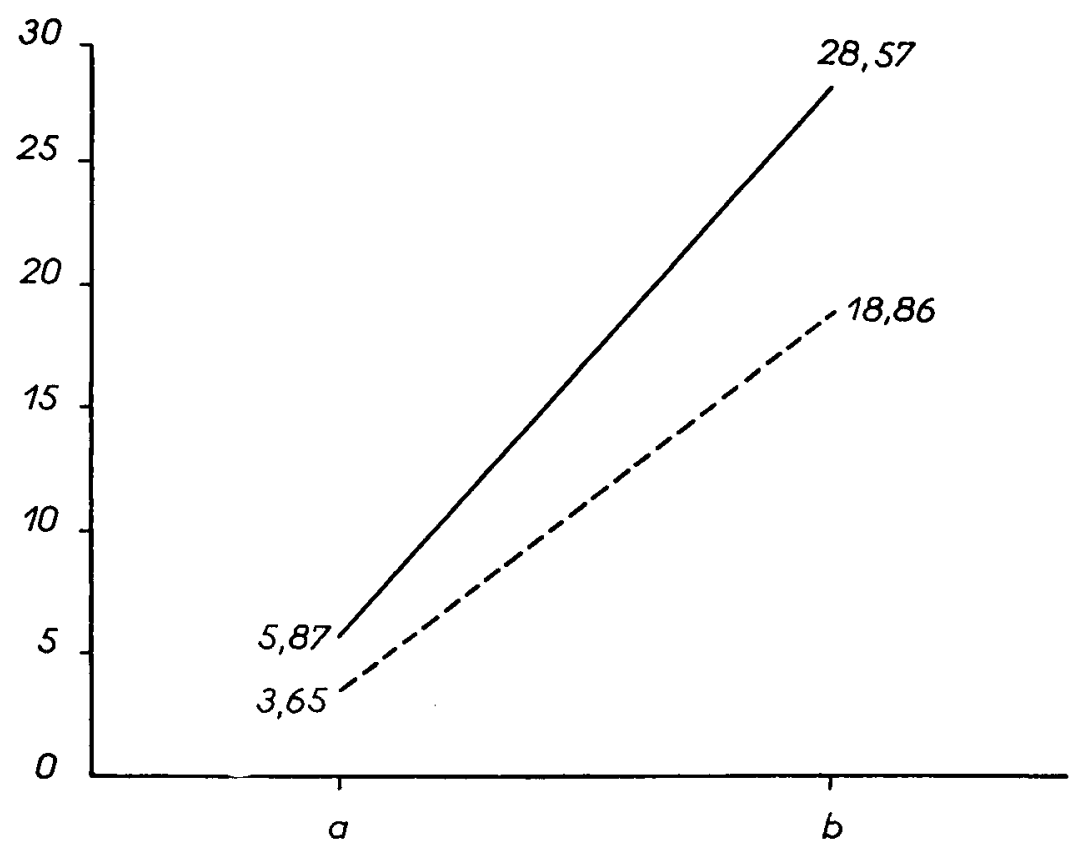

FIG. 40 - Comparaison entre l'action antibiotique des pollens stockés par l'abeille et par Méliponula bocandei.

- épreuve Proteus vulgaris.

- - épreuve B. Subtilis.

$a$ : pollen récolté par Apis mellifica.

$b$ : pollen récolté par ilétiponula bocandei.

gnons. On doit noter la différence d'activité entre la valeur antibiotique des deux extraits. Les trigones étant morts pendant l'hiver, il ne m'a pas été possible de faire de nouveaux prélèvements et de savoir si un pollen européen récolté et stocké par ces insectes aurait des propriétés antibiotiques différentes. En tout cas les trigones récoltaient très facilement des pollens divers dès leur établissement au laboratoire de Bures.

En résumé, les substances antibiotiques de la colonie de Trigona bocandei, si l'on excepte le pollen, sont en général moins actives que celles présentes chez Apis mellifica, deux fois moins dans l'épreuve sur Bacillus 
subtilis (voir figure 4I). Il résulte de ces essais que le nid de Trigona bocandei comme celui de l'abeille, renferme des antibiotiques et que 1'insecte lui-même n'est pas dépourvu d'un tel moyen de protection.

Je n'ai pas obtenu de résultats positifs avec l'extrait alcoolique de Meliponula quadrifasciata que m'avait expédié du Brésil NoGuEIRA Neto. Cependant, je ne puis conclure à l'inactivité antibiotique de ces insectes, car l'échantillon était vraiment trop restreint pour me permettre des essais suffisants.

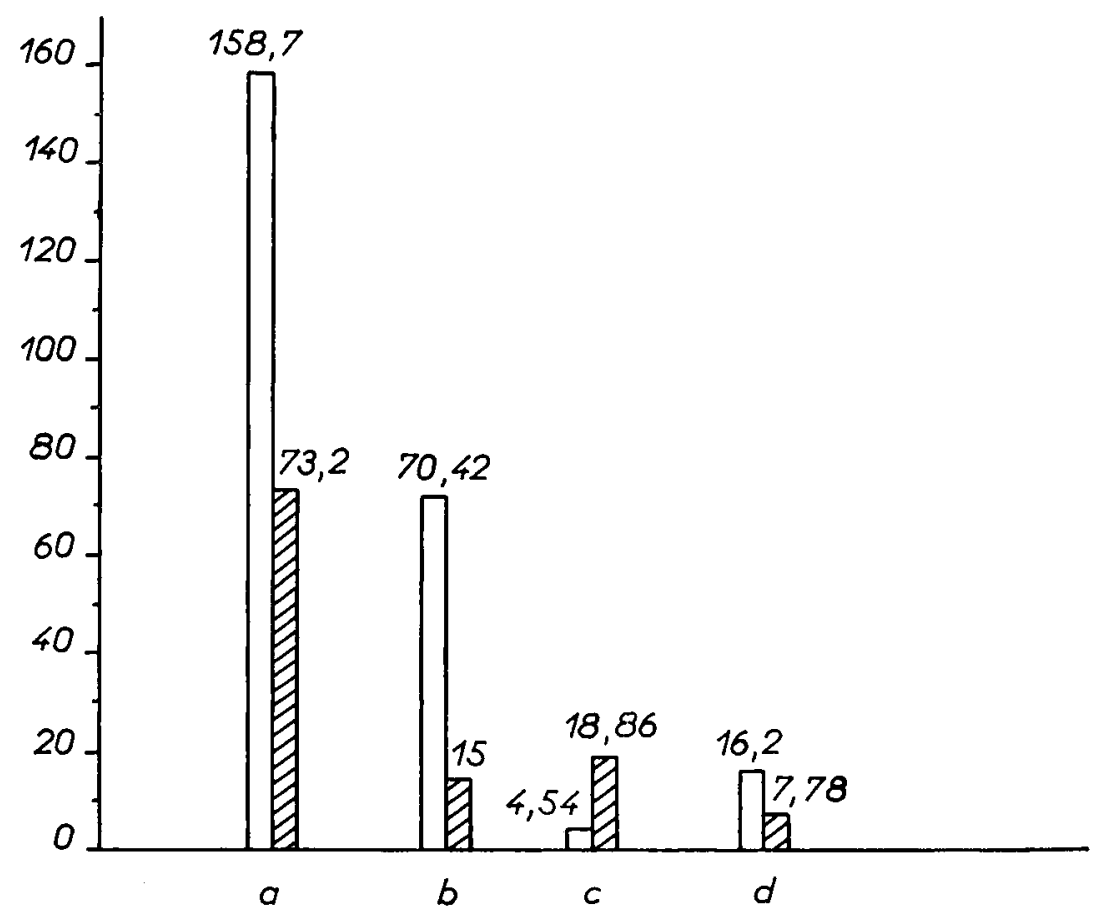

Fig. 4I. -- Comparaison de la valeur antibiotique de quelques substances provenant de la ruche d'Apis mellitica et de la colonie de Meliponula bocandei (épreuve B. Subtilis).

I... extraits d'abeilles.

$|i / / \bar{l}|$ extraits Mélipones.

$a$ : propolis.

$b$ : cire.

$c$ : pollen stocké en rellules.

$d$ : les insectes eux-mêmes.

\section{$2^{\circ}$ Les antibiotiques chez les bourdons.}

L,es Bombus hortorum et terrestris ont été extraits par l'alcool à chaud. I,a substance obtenue est active sur Bacillus subtilis $(\mathrm{U}=6,02)$ et sur Proteus vulgaris. Comme chez l'abeille, l'extrait est plus inhibiteur sur Proteus que sur subtilis. Les constituants du nid et le pollen stocké n'ont pas été essayés. L'antibiotique présent chez les bourdons a été éprouvé 
sur d'autres souches (voir tableau XXVI), mais il n'est actif, en dehors des deux bactéries déjà citées, que sur Salmonella pullorum $\mathrm{n}^{0} 309$. Dans tous les autres cas, il n'y a pas d'inhibition. L'activité sur Bacillus subtilis est voisine de celle de l'extrait de trigones.

\section{$3^{\circ}$ Les antibiotiques chez les guêpes et les frelons.}

La substance extraite de Vespa germanica ne s'est pas révélée inhibitrice $d u$ développement de Bacillus subtilis.

Au contraire, l'extrait alcoolique de Vespa crabro s'est révélé d'une activité voisine de celle des trigones et des bourdons ( $\mathrm{U}=7,74$ sur Bacillus subtilis). Cette substance s'est montrée très active sur Proteus vulgaris $(\mathrm{U}=\mathrm{I} 7,3)$ et légèrement inhibitrice vis-à-vis de Escherichia coli Bordet $(\mathrm{U}=5,2)$.

Il est curieux de constater que des insectes aussi voisins et de mœurs aussi semblables possèdent des moyens très différents de lutte envers les microorganismes. Cependant nous avions vu que ces deux hyménoptères, l'un comme l'autre, étaient peu sensibles aux mutilations. Que ces insectes diffèrent de l'abeille en ce qui concerne la présence de substances antibiotiques, cela semble normal puisque leur nutrition et leur cycle de développement est totalement différent. Les bourdons et principalement les mélipones, au contraire, s'en rapprochaient beaucoup plus. PAvaN qui a essayé l'action des extraits de Vespa crabro avait montré que l'ouvrière était plus ou moins active sur Escherichia coli et que la larve était inactive sur cette souche.

\section{$4^{\circ}$ Les antibiotiques chez Formica rufa.}

Je n'ai pas fait d'études très poussées sur les fourmis, ces insectes ayant fait l'objet des recherches de Pavan. En ce qui concerne Formica rufa PAVAN a éprouvé séparément l'abdomen, le thorax et la tête. Il trouve que seul l'abdomen possède des propriétés antibiotiques vis-à-vis de Escherichia coli et Proteus vulgaris. De même l'ensemble de ses travaux sur Iridomyrmex lui a fait supposer que la substance active était produite par des glandes anales. Je n'ai pas cherché à savoir la provenance de cette substance chez la fourmi rousse. J'ai simplement éprouvé des extraits alcooliques de l'insecte adulte entier sur quelques souches : l'extrait est très actif sur Bacillus subtilis souche Caron $(\mathrm{U}=54,64)$, sur Salmonella gallinarum, E. Coli Bordet, Pseudomonas pyocyanea et principalement sur Proteus vulgaris (voir figure 38 et le tableau XXVI). Je dois préciser que j'ai trouvé des différences allant dı simple au double entre des insectes capturés fin mars et ceux capturés vers le 15 mai, ces demiers contenant plus d'antibiotique. D'autre part, l'extrait de 
nymphes n'est jamais actif. Ces résultats concordent avec ceux de Pavan sur Iridomyrmex, cet auteur ayant montré que seuls les adultes sont antibiotiques à l'exclusion des larves, des nymphes et des ouvrières naissantes. Les fourmis doivent être sorties du nid et pigmentées pour que la substance antibactérienne apparaisse et enfin, la quantité d'iridomyrmécine est variable suivant le stade physiologique, la date du prélèvement et l'emplacement des nids (PAvan). Cependant, je n'ai jamais trouvé d'action avec 1'extrait de Formica rufa sur les Escherichia coli no ${ }^{\circ}$, I3, I6, du tableau I. En résumé, les colonies de fourmis sont également défendues contre les infections par des substances antibiotiques dont le comportement est similaire de celles extraites chez Apis mellifica.

\section{$5^{\circ}$ Les substances antibiotiques chez les termites.}

Ayant trouvé des substances antibiotiques dans la plupart des sociétés d'insectes, j'ai pensé que les termites devraient posséder les mêmes substances. I a difficulté de s'en procurer en quantité suffisante pour les épreuves a limité mes recherches. J'ai essayé les ouvriers de deux espèces de termites, le Reticulitermes lucifugus du Sud-Ouest français et une autre espèce africaine non déterminée. Ces isoptères ont été soumis à une extraction alcoolique à chaud suivant les méthodes habituelles. L'extrait de Reticulitermes lucifugus est très actif sur Bacillus subtilis $(U=46,72)$ c'est-à-dire d'une valeur antibiotique voisine de celle des fourmis. Par contre l'extrait de termites d'origine africaine (indéterminés) s'est révélé totalement inactif même avec $\mathrm{I} / 4 \mathrm{O}$ d'extrait sec dans le milieu de culture. Le facteur antibiotique thermostable trouvé chez Reticulitermes lucifugus s'est montré en outre actif sur Pseudomonas pyocyanea, Escherichia coli Bordet, Salmonella gallinarum $n^{0} 38$ et Salmonella pullorum $n^{0} 309$ et complètement inactif sur la plupart des Escherichia coli (voir tableau XXVI) ; PAVAN a éprouvé également la valeur antibiotique des termites ; il a montré l'inactivité de l'extrait de soldats et d'ouvrières de Calotermes flavicollis $F$. sur une douzaine de souches bactériennes dont Escherichia coli. On ne peut rien déduire de ces essais très fragmentaires. Les termites mériteraient à cet égard une étude très approfondie étant donné leur importance et leur diversité. I1 faudrait bien également éprouver les matériaux de leur nid et leurs provisions. 


\section{LES ANTIBIOTIQUES CHEZ LES AUTRES INSECTES}

Après avoir montré la présence des principes antibiotiques chez divers insectes sociaux appartenant à des familles diverses, $j$ 'ai étudié également quelques insectes à caractère non social de différents groupes : apides solitaires, homoptères vivants groupés, lépidoptères, coléoptères, diptères, dyctyoptères, dermaptères et planipennes.

\section{I $^{\circ}$ La substance antibiotique chez un apide solitaire (Xylocopa violacea Latr.).}

Un extrait alcoolique des xylocopes a été essayé sur Bacillus subtilis souche Caron. Cet extrait est très actif, il correspond à $U=23,8$ et son action est plus importante que chez les trigones, guêpes, bourdons et abeilles. Ce facteur antibiotique des xylocopes doit être très stable à la température du laboratoire, car des insectes prélevés dans une collection entomologique où ils étaient conservés depuis plusieurs années se sont révélés d'une activité inhibitrice semblable à celle des animaux récemment tués. La quantité limitée de sujets que j'ai pu me procurer ne m'a pas permis d'étudier l'action de la substance sur d'autres souches bactériennes. Pavan a déterminé également la valeur antibiotique de cet apide solitaire sur une dizaine de bactéries. Il a séparé les extraits de tête, de thorax et d'abdomen de mâles. Ses résultats sont négatifs (notamment sur Escherichia coli), mais l'explication est peut-être facile à trouver : chez l'abeille, nous avons vu que les extraits de mâles ne sont jamais antibactériens.

\section{$2^{\circ}$ La valeur antibiotique des extraits d'Homoptères.}

Le Pyrrhocoris apterus est un homoptère que l'on rencontre vivant en colonies assez importantes au pied de certains arbres tels que les tilleuls ou les marronniers. Bien que nous n'ayons pas affaire à des insectes sociaux, il était intéressant de savoir si un groupement important d'individus de tous âges en un point donné, possédait un moyen de défense biochimique envers les infections microbiennes. J'ai à cet effet extrait par l'alcool à chaud une dizaine de grammes de ces insectes (de tous les âges). Cet extrait repris en milieu aqueux et éprouvé sur Bacillus subtilis et. 
Proteus vulgaris n'a jamais donné de réultats positifs, même avec des concentrations très fortes d'extrait ( $1 / 25$ environ). Ces insectes quoique vivant en colonies assez importantes, ne possèdent pas de substances antibiotiques pour lutter éventuellement contre une épizootie.

\section{$3^{\circ}$ Les substances antibiotiques chez les lépidoptères.}

L'épreuve a été réalisée au moyen d'un extrait alcoolique à chaud de Arctia caja $L$. capturés grâce à un piège lumineux. La substance extraite de ces lépidoptères est très antibiotique vis-à-vis de Bacillus subtilis $(\mathrm{U}=30$, I2) et encore plus $(\mathrm{U}=60,24)$ vis-à-vis de Proteus vulgaris. Au contraire, une conservation au réfrigérateur pendant trois mois est suffisante pour détruire presque la totalité de ce principe antibiotique. Cependant il est encore possible de montrer une action inhibitrice partielle sur Salmonella gallinarum $\mathrm{n}^{0} 47$, Escherichia coli Bordet. Mais je n'ai jamais pu trouver d'action antibiotique sur Pseudomonas pyocyanea. On peut dire qu'il existe chez Arctia caja un principe antibiotique thermostable (il résiste ${ }_{5}$ minutes à ${ }^{2} 20^{\circ}$ ), mais que ce facteur se dégrade en fonction du temps. Il est inutile de rappeler à nouveau les travaux de Kondsumi sur les antifongiques présents chez les chenilles de lépidoptères, je les ai suffisamment analysés dans la première partie de cette thèse. Pavan en revanche, n'a pas trouvé d'action antibiotique chez Cossus cossus I., Thaumetopoea pityocampa Sch. et Sitotroga cerealella Oliv. vis-àvis d'une dizaine de souches bactériennes.

\section{$4^{\text {o }}$ Les substances antibiotiques chez les coléoptères.}

L'extrait alcoolique à chaud a porté sur un lot de Cerambycidae, principalement Cerambyx cerdo et Cerambyx scopolii. La liqueur évaporée et reprise par l'eau s'est montrée antibiotique vis-à-vis de Bacillus subtilis $(\mathrm{U}=20)$ et de Proteus vulgaris $(\mathrm{U}=40)$. Ce facteur antibactérien est thermostable. PAVAN qui a éprouvé des extraits d'une douzaine de coléoptères a trouvé actifs seulement les extraits du genre Brachinus et de Paederus fuscipes Curt. Ce dernier Staphylinide est antibiotique vis-àvis de Mycobacterium phlei. Des extraits de Brachinus sont actifs sur une dizaine de bactéries diverses. Au contraire PAvan n'a pas obtenu de résultats positifs avec les coléoptères vésicants tels que Lytta vesicatoria I. et Meloe cicatricosus $1 \mathrm{ch}$. I a répartition de la présence de facteurs antibiotiques semble également chez les coléoptères être très variable. I a présence de principes antibactériens n'est pas attachée à tel groupe ou genre et en l'état actuel de la question il est difficile de se faire une opinion sur l'ensemble. J'en terminerai avec les coléoptères en rappelant la grande activité antibactérienne et insecticide trouvée par PAVAN (I953) chez la larve de Melasoma populi. 


\section{$5^{\circ}$ Les substances antibiotiques chez les diptères.}

Nous avons vu dans les généralités de cette troisième partie quelle était l'importance des substances antibiotiques chez les larves de diptères et principalement les diptères myasogènes. Cependant, si l'accord est fait sur la présence de substances antibactériennes chez les larves de ces derniers, nous allons voir que leur répartition n'est pas générale. PAvaN a montré que les extraits de larves de Sarcophaga sp. étaient inactifs sur 8 souches bactériennes éprouvées. Nous savons que Musca domestica est parfois naturellement immune vis-à-vis d'un champignon pathogène Empusa muscae (PAILLOT I933). D'après mes premières expériences, je savais que des mouches adultes plongées dans un milieu nutritif ne donnaient pas lieu à culture de microorganismes dans tous les cas. L'essai avec Drosophila melanogaster adulte m'avait donné les mêmes indications. C'est alors que j'ai patiqué l'extraction alcoolique à chaud sur un échantillon de Musca domestica. Cet extrait en milieu aqueux s'est révélé totalement inactif sur Bacillus subtilis. Sur Proteus vulgaris, je n'ai pas obtenu une inhibition totale, mais simplement une action retardatrice sur le développement ( 24 heures de retard par rapport aux témoins). La mouche domestique possède-t-elle un principe antibiotique sur la cuticule? Les expériences n'ont pas tranché la question, cependant si une telle substance existe, elle est peut-être très labile ou spécifique de certaines bactéries. L'expérience qui consiste à mutiler les insectes (par ablation des pattes et des antennes) a montré une très grande résistance des animaux dont la survie est semblable à celle des témoins.

\section{$6^{\circ}$ Les substances antibiotiques chez les Dictyoptères.}

L'extraction alcoolique à chaud a été effectuée sur Blatella germanica. I'extrait obtenu est évaporé et repris par l'eau. L'épreuve de cette substance sur Bacillus subtilis se révèle négative, cependant à une certaine concentration on obtient un retard de 24 heures dans le développement de la bactérie par rapport au témoin. I'inhibition complète n'a pas été observée. Les essais sur plusieurs autres souches ont été négatifs et seules quelques Salmonella ont réagi à l'addition de cet extrait dans le milieu de culture (voir tableau XXVI).

\section{$7^{\circ}$ Autres insectes recélant un antibiotique.}

Nous avons vu dans les généralités que si l'on plonge dans un milieu les deux insectes suivants, il ne s'y développe aucun microorganisme.

- Chrysopa perla (Planipenne).

- Forficula auricularia (Dermaptère).

Jen'ai pas pratiquél'extraction de ces deux insectes pour mesurer leurvaleur antibiotique car je ne possédais pas assez d'exemplaires de ces animaux. 


\section{CONCLUSION DE LA TROISIÈME PARTIE}

\section{Io Interprétation des résultats obtenus chez les insectes divers.}

L'examen des résultats concernant la valeur antibactérienne d'extraits des divers insectes ne m'a pas permis de dégager des règles générales. Les figures 38,42 et le tableau XXVI foumissent une vue d'ensemble de ces essais.

a) Tout d'abord, il n'est pas possible de trouver des correspondances valables entre deux insectes même voisins ; par exemple, nous avons montré la différence existant entre deux animaux du même genre tels que Vespa germanica et Vespa crabro. De même, chez les isoptères, certains termites recèlent une substance antibiotique très active et les autres, pas du tout. Chez les diptères, il y a également de grandes différences. En revanche il existe des rapports entre des insectes éloignés comme Reticulitermes lucifugus et Formica rufa. Citons encore l'inactivité des extraits de certains insectes sociaux comme Vespa germanica, les termites africains et un homoptère vivant en groupe comme Pyrrhocoris apterus. Mais l'extrait d'un apide isolé comme $X y$ locopa possède la même action que celle des Apides ou Vespides sociaux tels que Trigona, Bombus, et Vespacrabro. Seule l'action des antibiotiques de la colonie $d$ 'A pis mellifica est comparable à celle de la colonie de Trigona bocandei sur Bacillus subtilis (voir figure 4I); malgré cela cette activité n'est pas la même sur d'autres souches bactériennes (figure 38). Cet ensemble de résultats montre une grande irrégularitédans la présence et l'action de substances antibiotiqueschezles insectes. Cependant, d'après l'ensemble des travaux actuels, on peut supposer qu'un grand nombre d'insectes, dans les ordres les plus divers sont dotés de substances antibiotiques.

b) Nous avons vu que différents auteurs ont montré que les larves s'infectent davantage que les insectes adultes (PAILLo' r933) et que la présence d'antibiotiques chez certains apparaissait lorsque 1'adulte atteint une certaine maturation physiologique (PAVAN I952). J'ai trouvé chez Apis mellifica des mécanismes semblables, mais ceci n'est pas du tout général chez les insectes. En effet, nous savons que les larves de diptères myasogènes et les drosophiles possèdent des propriétés antibiotiques, et j'ai montré dans ce qui précède que les adultes de mouches domestiques et de drosophiles ne contenaient pas de principe antibiotique. Je ne puis donc pas faire entrer en jeu d'une manière générale, les facteurs ayant trait au stade ou à l'âge des insectes dans la production de telles substances.

c) De même je supposais que les insectes sensibles à des mutilations possédaient à la surface de leur corps des substances antibiotiques et que les autres possédaient un mécanisme de défense antibactérienne très différent. Les expériences préliminaires n'ont pas confirmé cette hypothèse et les insectes sensibles aux blessures ne recèlent pas forcément plus de facteur antibactérien que les autres. En effet, les frelons et les guêpes sont peu sensibles aux mutilations et j'ai déjà montré ci-dessus que le premier 
possédait un antibiotique tandis que le second en était dépourvu. Cependant, d'une manière générale, les auteurs admettent que la présence d'insectes prédateurs favorise les épidémies chez les insectes, ces prédateurs favorisant la pénétration des microorganismes pathogènes dans le sang par les blessures qu'ils occasionnent. La barrière cuticulaire est de toute évidence d'une utilité primordiale chez les insectes, du point de vue microbien.

d) Tous les essais portant sur les antibiotiques ou antifongiques chez les insectes, sauf les exceptions concernant Iridomyrmex et Bombyx mori, ne sont pas encore assez précis pour en tirer des conclusions intéressantes. Pavan lui-même a passé en revue un grand nombre d'insectes mais il ne fait état d'aucune mesure d'activité antibiotique par rapport au poids du matériel d'origine. Il s'ensuit que nous sommes encore à la période de défrichage et que tous ces essais devraient être repris en tenant compte du poids d'insectes, du solvant employé pour l'extraction, du stade et de 1'âge, du sexe, de la saison de prélèvement; et de la sensibilité des souches bactériennes choisies, autant de facteurs qui font varier dans de grandes limites la valeur des résultats.

Si l'on vetut tirer quelques conclusions de cet ensemble préliminaire on peut dire que :

- La majorité des insectes sociaux possèdent une substance antibiotique, ainsi que les matériaux de leurs nids.

- Les apides sociaux et au moins un apide solitaire possèdent une substance antibiotique.

- Les lépidoptères, les coléoptères et les hyménoptères semblent posséder des facteurs antibiotiques plus actifs que ceux des autres insectes.

- L'action sur les souches de Proteus est toujours importante sur tous les insectes essayés; tandis que l'activité antibiotique vis-à-vis des Escherichia coli est toujours faible ou nulle.

\section{$2^{\circ}$ Comparaison de l'action antibiotique entre les divers insectes et l'abeille.}

J'ai recherché les similitudes et les divergences entre $A$ pis mellifica et les autres insectes. Le tableau XXVI et les figures 38 et 42 résument les résultats. I,es comparaisons sont extrêmement difficiles et demandent beaucoup de prudence.

Tout d'abord aucun extrait d'insectes (même chez les apides) ne s'est révélé actif sur les Escherichia coli, tandis que le facteur antibactérien de l'abeille inhibe cette souche. Au contraire, les extraits de frelons, de fourmis, de bourdons, de lépidoptères et de coléoptères sont très actifs sur Proteus comme celui de l'abeille. Seuls les extraits de trigones sont peu actifs sur Proteus (U proteus $=\mathrm{U}$ subtilis). Il est assez curieux de constater que Vespa crabro exerce une action très voisine de celle de Apis 
mellifica. Cependant, si ces deux hyménoptères se ressemblent par leur caractère social, ils diffèrent par beaucoup de points, ne serait-ce que par leur régime alimentaire. On peut dire, d'autre part, que Trigona bocandei apide voisin de l'abeille diffère de celle-ci au point de vue de l'activité antibiotique. Les extraits de nombreux insectes n'ont pas été essayés sur toutes les souches, ce qui nous gêne beaucoup pour l'interprétation des résultats. La comparaison avec Formica rufa est difficile, car suivant la saison la

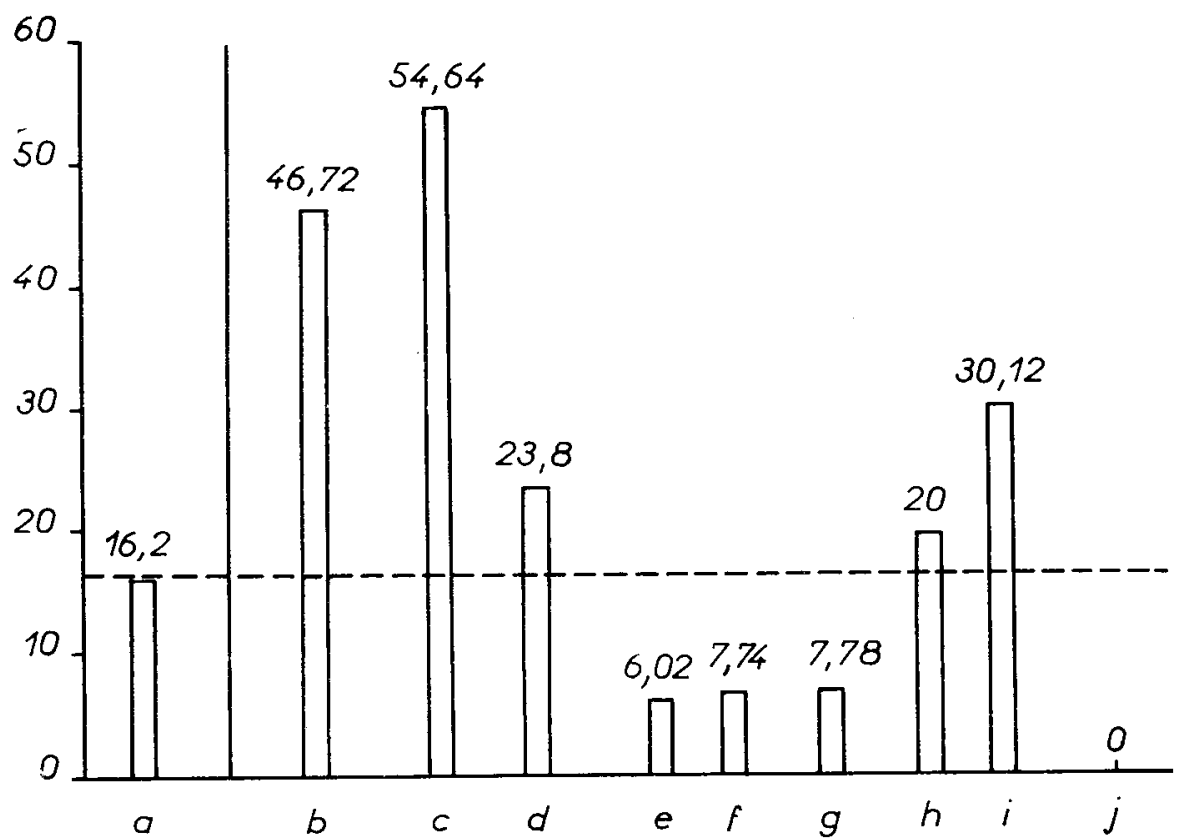

Fig. 42. - Comparaison de la valeur antibiotique des extraits d'abeilles et ceux de quelques antres insectes (épreuve B. subtilis).

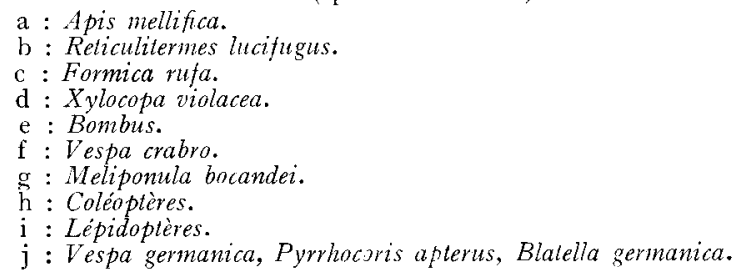

valeur antibiotique de l'extrait varie. Cependant, l'activité inhibitrice est toujours plus grande pour Proteus vulgaris que pour Bacillus subtilis comme chez l'abeille. De mêtne chez Bombus, je retrouve ce même phénomène.

Il serait intéressant d'étudier la valeur antibiotique des matériaux et des provisions des nids d'insectes sociaux et, par exemple, le pollen stocké par les Bombus, pour voir si ces matériaux sont antibiotiques, comme dans la colonie d'abeilles. D'autre part, nous avons vu des ressemblances quant à l'influence de l'âge, du stade, et de la maturation physio- 
logique entre l'abeille et les fourmis. Au contraire, l'abeille diffère de certains insectes par l'absence de substances bactéricides dans son sang, et l'absence de substances antifongiques dans son corps. Mais l'abeille est moins sujette aux maladies à champignons que certains insectes et dans la ruche, la propolis joue le rôle d'antifongique.

\section{$3^{\circ}$ Signification et utilisation possible des substances antibiotiques chez les insectes.}

La lutte biochimique que mènent les êtres vivants contre les microbes pathogènes est renforcée par la présence de principes antibiotiques chez les insectes. I1 semble de plus en plus que ce mode de défense de l'individu soit beaucoup plus répandu qu'il ne le paraissait il y a quelques années. D'HERELIE (IgII) par exemple avait déjà remarqué que dans la nature 20 à $25 \mathrm{p}$. Ioo des criquets résistent à Coccobacillus acridiorum et présentent une immunité naturelle lors des grandes épizooties. Ces phénomènes de résistance sont très fréquents et un certain pourcentage d'entre eux paraît dî̀ à l'immunité biochimique. D'autre part, les principes antibiotiques extraits des insectes sont plus ou moins spécifiques. MFTALNIKOV (I927) a montré par exemple la grande virulence à faibles doses de certains bacilles sur Galleria mellonella (E. coli, B. subtilis, $B$. proteus) tandis que ce lépidoptère résiste très bien au bacille de Koch. On peut supposer également que certains insectes possèdent des facteurs inhibiteurs très sélectifs, lorsque l'on considère leur écologie. C'est le cas, par exemple, des coprophages dont les boulettes stercoraires se conservent très bien et des nécrophages qui ne sont jamais atteints par les infections microbiennes. Il est probable également que les insectes vecteurs des bactéries pathogènes et très spécialisés sont résistants à ces mêmes bactéries; qui sait si cette résistance n'est pas due à une substance spécifique?

La connaissance de l'origine et du mécanisme d'action de ces principes antibactériens pourrait aider dans une large mesure l'entomologie appliquée. L'étude de l'apparition de l'inhibiteur en fonction du stade ou de l'âge d'un insecte permettrait dans la lutte biologique d'appliquer avec plus de chances de réussite tel ou tel agent pathogène à tel ou tel moment. De même la protection des insectes utiles tels que l'abeille, et le ver à soie pourrait se pratiquer d'une manière plus rationnelle et se réduirait peut-être simplement à une question d'alimentation.

Enfin, ces antibiotiques extraits des insectes ont peut-être un intérêt thérapeutique pour 1'homme et les animaux supérieurs. On a montré par exemple l'action inhibitrice in vitro d'extrait de Paederus fuscipes et de Brachinus sur Mycobacterium phlei. D'autres extraits de divers insectes se sont révélés actifs in vitro sur $B$. anthracis, S. paratyphi, S. typhi. Citons encore l'utilisation effective des larves myasogènes dans la stérilisation et le nettoyage des plaies. 


\section{CONCLUSIONS GÉNÉRALES}

\section{A. - Importance de la défense biochimique.}

La présence des substances antibiotiques chez l'abeille et chez les insectes confirme une fois de plus l'existence d'une défense biochimique des êtres vivants vis-à-vis des infections microbiennes. Nous avons vu que GLASER avait pressenti, il y a déjà plus de quarante ans l'importance d'un tel moyen de lutte antimicrobien chez les insectes. Cependant, dans ce domaine c'est PaILLot qui a montré par de multiples exemples et établi d'une manière irréfutable que la phagocytose n'était pas le moyen unique de protection chez l'insecte contre les microbes pathogènes. Actuellement, depuis quelques années, les études concernant la défense biochimique chez les animaux ont pris une grande importance. La théorie des réactions cellulaires d'immunité, chère à METchnikofF et longtemps considérée comme la seule valable, ainsi que celle des réactions humorales se trouvent maintenant complétées par d'autres notions dont l'importance va grandissant chaque jour. Les travaux de Nutini, Bloom, puis Hirsch et Dubos sur les mammifères et l'homme sont parmi les plus représentatifs et ouvrent une voie nouvelle à la médecine. Dans un domaine plus modeste, les zoologistes ont apporté leur contribution à cette théorie toute neuve. Je rappellerai simplement ici les noms de PAVAN et de KoIDsumi. J'ai montré, de mon côté, l'existence d'un tel mode de défense chez l'abeille et chez quelques insectes. L'étude de telles substances chez Apis mellifica avait à mon avis un intérêt certain. En effet, il s'agit là d'une société d'insectes où la lutte contre les épizooties a une importance considérable à cause des facteurs multiples qui favorisent les infections (population dense d'individus, température et hygrométrie constante, etc.). J'ai donc précisé, en ce qui me concerne, que l'abeille emprunte largement les moyens de défense qui lui sont nécessaires aux substances antibiotiques présentes dans la ruche. Ces recherches confirment dans une certaine mesure et dans un domaine nouveau l'importance de cette résistance biochimique pour de nombreux êtres vivants.

\section{B. - La présence de substances antibiotiques dans la colonie d'abeilles confirme les réactions d'équilibre constant qui règlent cette société d'insectes.}

Nous savons que, dans la ruche, il existe tout un système de régulations entre diverses activités de la colonie et que rien ne s'y fait individuellement. L'on peut dire également d'après les recherches que j'ai effectuées que la sécrétion ou la récolte de substances antibiotiques dans la ruche semblent réglées de la même façon. Il existe un équilibre dans la ruche qui contrebalance l'influence de facteurs divers tels que 
1a nutrition, la saison, le développement du couvain, l'apparition d'une maladie, etc. Le dégorgement continuel de la gelée royale aux larves en fournit un bon exemple. De même l'échantillon moyen de pollen récolté par les abeilles montre une valeur antibiotique toujours à peu près semblable pour une période donnée. Nous avons vu également l'interaction qui existe entre la reine et les ouvrières sur la production de leurs antibiotiques respectifs. Je rappellerai à nouveau les rapports existant entre l'âge de l'ouvrière, le développement des glandes pharyngiennes et sa production d'antibiotique et de même chez la reine le développement des glandes mandibulaires et de l'action antibiotique en fonction de l'âge. Enfin, avant d'en terminer avec cette question, il est très important de rappeler que seule la propolis est antifongique, mais qu'elle est présente partout dans la colonie et que d'autre part les champignons pathogènes sont plus rares chez l'abeille que chez d'autres insectes. $L a$ production ou la récolte d'antibiotique et d'antifongique dans la colonie d'Apis mellifica est bien le résultat d'un ensemble de réactions équilibrées, au même titre que les autres activités de cet insecte.

\section{C. - Importance des antibiotiques des insectes en agronomie.}

Une connaissance plus approfondie de la sécrétion de substances antibiotiques chez les insectes pourrait rendre de grands services en agronomie.

I ${ }^{\circ}$ Dans la défense des insectes utiles contre les épizooties, notamment chez Bombyx mori et Apis mellifica. Chez l'abeille, il est certain que l'ensemble des substances antibiotiques que $j$ 'ai étudiées agissent dans une certaine mesure sur les souches pathogènes. Une étude plus poussée de l'alimentation des larves par exemple permet peut-être d'envisager la lutte contre certaines maladies sous un jour nouveau. La propolis dont 1'action antifongique est très intense pourrait également expliquer l'absence ou le développement des infections fongiques. D'un autre côté, la conservation $d u$ pollen dans les ruches, grâce à un facteur antigerminatif puissant, est d'une grande importance en apiculture.

$2^{\circ}$ Dans la lutte biologique contre les insectes nuisibles et principalement lors de l'emploi de bactéries pathogènes, il serait d'un intérêt capital d'envisager chaque insecte à part afin de savoir à quel stade ou à quelle époque il serait le plus vulnérable. Cette étude du mécanisme des défenses biochimiques chez l'insecte donnerait certainement des résultats intéressants. Déjà PAILLOT en Ig24 disait que "l'immunologie comparée " des insectes favoriserait la réussite dans les essais de lutte biologique microbienne en agronomie. Je pense que la présence assez fréquente de substance antibiotique chez les insectes justifie maintenant des études dans ce sens pour améliorer les résultats obtenus dans la lutte biologique microbienne en entomologie agricole. 


\section{D. - Importance des antibiotiques présents chez les insectes en thérapeutique.}

Nous avons vu que de nombreux auteurs se sont intéressés à 1'utilisation possible des extraits d'insectes ou de leurs produits contre les bactéries pathogènes. Parmi les insectes les plus étudiés à ce point de vue citons Iridomyrmex humilis, les larves de diptères myasogènes, Paederus fuscipes, Melasoma populi et les produits de l'abeille tels que le miel, la propolis, la gelée royale, le pollen. Les extraits d'abeilles entrent à peine dans le cycle des essais in vivo. Le pollen en revanche, a déjà donné des résultats en rapport avec les essais in vitro. Les souches bactériennes pathogènes éprouvées in vitro avec les extraits d'insectes sont principalement le bacille tuberculeux, le bacille diphtérique, les Entérobactériacées, les Proteus. Les antibiotiques extraits des insectes ou leurs produits que j'ai essayés sont en général très actifs sur Bacillus Proteus. I,es produits de l'abeille se révèlent intéressants, non toxiques, et actifs sur de nombreuses souches. L'abeille nous offre une grande facilité de récolte et d'extraction de tous ses produits et l'animal lui-même est facile à obtenir en grande quantité, tandis que certains autres insectes poseraient des problèmes complexes d'élevage. L'apiculture moderne permettrait le cas échéant de fournir le matériel brut voulu à la médecine sans adaptation préalable. Mais cette étude de la valeur thérapeutique des produits de la ruche dépasse le cadre de mon travail et j'espère que d'autres chercheurs voudront bien s'y intéresser. De toute manière, 1'homme dispose encore de bien peu d'antibiotiques d'origine animale et je pense que, dans cette voie, il est possible d'en découvrir qui soient intéressants pour la thérapeutique.

\section{E. - Confirmation des travaux antérieurs.}

Cette étude m'a permis de confirmer les travaux ou les suggestions de différents auteurs. Tout d'abord, PAvan avait vu justeendressant un inventaire des antibiotiques d'origine animale et en étudiant plus spécialement les insectes. Les recherches de WHITE gardent également toute leur valeur depuis 50 ans et représentent un travail de base important. Enfin, j'ai pu vérifier scientifiquement la valeur d'un certain nombre d'utilisations empiriques des produits de la ruche, tels que la valeur antigerminative de certains d'entre eux pour les grains de végétaux supérieurs. L'examen des fractions actives nous ramène très souvent à des groupements chimiques qui "inhibent la vie " (inhibiteurs de germination des pollens et des graines, antibiotiques ou antifongiques) que l'on retrouve aussi bien chez les végétaux que chez les animaux. 


\section{RÉSUMÉ}

I. - Le corps de l'abeille adulte renferme une substance antibiotique hydrosoluble et alcoolosoluble. Cet extrait n'est pas toxique pour la souris. La substance est thermostable et l'extrait est stable s'il est conservé à $0^{\circ} \mathrm{C}$.

2. - L'antibiotique de l'abeille est répandu à la surface de la cuticule. $\mathrm{L}_{\text {a }}$ tête recèle plus d'antibiotique que le thorax et le thorax plus que l'abdomen. Le venin a une action peu importante et le sang a une action nulle.

3. - Chez l'ouvrière les glandes pharyngiennes sont douées de pouvoir antibiotique, tandis que les glandes mandibulaires sont sans action sur les bactéries.

4. - L'extrait de mâles est toujours inactif. L'extrait de reines est actif, mais différent de celui de l'ouvrière. Son activité varie en fonction de l'âge et de la présence des ouvrières. Le contact des ouvrières favorise la production d'antibiotiques chez la reine. $\mathrm{I}_{\uparrow}$ a sécrétion des glandes mandibulaires de la reine est antibiotique, mais celle des glandes pharyngiennes ne l'est pas.

5. - L'antibiotique chez les ouvrières est plus abondant si celles-ci sont privées de reine. La sécrétion dépend également de l'âge et de l'état physiologique des glandes pharyngiennes, de la saison, du régime alimentaire (protéique), de la race, de l'origine géographique et de l'état pathologique de la colonie d'abeilles.

6. - Les larves, les nymphes et les insectes naissants ne possèdent pas de facteur antibiotique, quelle que soit leur caste.

7. - L'antibiotique de l'abeille se trouve dans la fraction des acides gras.

8. - L'antibiotique de 1'abeille est actif sur 30 souches bactériennes étudiées. Son activité est très intéressante vis-à-vis des Salmonella, de Proteus vulgaris et Bacillus alvei.

9. - La substance antibiotique extraite de l'abeille n'est pas antifongique. Par contre elle inhibe très fortement la germination des pollens. De même, cet extrait inhibe partiellement la germination des graines.

Io. - La propolis possède une substance antibiotique, hydrosoluble et alcoolosoluble, thermostable et stable au vieillissement. L'origine de cette substance provient de la résine récoltée sur les bourgeons de peuplier qui fournit un extrait dont l'action est similaire. Cette substance n'a pas d'action inhibitrice vis-à-vis des Escherichia coli et de Pseudomonas pyocyanea, elle est an contraire très active sur Proteus vulgaris et Bacillus alvei. $\mathrm{I}_{\mathrm{A}}$ propolis possède en outre des propriétés antigerminatives puissantes vis-à-vis des grains de pollen et des graines de végétaux supérieurs. 
L'extrait de propolis est d'autre part le seul antifongique puissant de la ruche.

II. - La cire possède une substance antibiotique hydrosoluble, mais principalement soluble dans l'acétone et thermostable. Son activité est nulle sur les Escherichia coli et le Pseudomonas pyocyanea et importante sur Proteus vulgaris, Salmonella gallinarum et Bacillus alvei. L'extrait de cire ne possède pas de facteur antifongique, mais inhibe partiellement la germination des graines.

I2. - Le pollen récolté par les abeilles contient un facteur antibiotique très actif. Ce facteur alcoolosoluble et hydrosoluble provient de la plante et de l'abeille. La valeur antibiotique du pollen augmente en fonction du temps et de la température au cours de sa conservation. Certains pollens sont inactifs sur les bactéries, màis un échantillon moyen de la récolte est toujours antibiotique. L'action est principalement nette sur les Salmonella, Proteus vulgaris, E. Coli Bordet, E. Coli B., Bacillus alvei et Bacillus larvae. De plus il est actif sur les 30 souches du tableau I. L'extrait de pollen a une faible action sur la germination du pollen et inhibe partiellement la germination des graines. Sa valeur antifongique est nulle.

I3. - Le miel contient une substance antibiotique thermostable et photolabile, extractible par 1'acétone. Cette substance provient en grande partie de l'abeille. L'activité de cet antibiotique est faible par rapport aux autres produits de la ruche; les souches les plus sensibles sont Proteus vulgaris et Bacillus alvei. Aucune action sur Escherichia coli et Pseudomonas pyocyanea. L'extrait de miel n'a pas de pouvoir antifongique. En revanche c'est un inhibiteur puissant de la germination des pollens et des graines.

I4. - La gelée royale possède un facteur antibiotique, soluble dans l'eau, l'alcool et l'éther. Cette substance est très instable et cependant résiste à $I 20^{\circ} \mathrm{c}$. pendant 20 minutes. C'est la fraction acide de la gelée qui est active. Le pouvoir antibiotique est faible vis-à-vis des autres produits de la ruche, nul sur quelques souches de Escherichia coli et Psendomonas pyocyanea, faible sur Bacillus larvae, plus actif sur $E$. Coli B, et Proteus vulgaris. Ira gelée royale n'est pas antifongique, mais elle inhibe partiellement la germination des graines et des pollens.

I5. - Aucun des produits de la ruche ne possède une substance antibiotique ayant des rapports étroits avec 1'antibiotique extrait du corps de 1'abeille.

I6. - Toutes les substances antibiotiques extraites des colonies d'abeilles sont actives (plus ou moins) sur les souches pathogènes du couvain.

I7. - Des substances antibiotiques se rencontrent chez d'autres insectes sociaux, mais ce n'est pas une règle générale. Chez Meliponula 
bocandei, on trouve des actions voisines de celles trouvées chez Apis mellifica (pour les insectes et leurs produits).

I8. - Un apide solitaire, Xylocopa violacea, possède également une substance antibiotique.

I9. - L a recherche de substances antibiotiques chez des insectes divers a montré l'existence ou l'absence de celles-ci dans divers ordres, sans qu'il soit possible d'en donner une explication.

\section{SUMMARY}

I. The body of the adult bee contains an antibiotic substance, soluble in water and in alcohol. This extract is non-toxic to mice. The substance is thermostable and the extract is stable if it is stored at $0^{\circ} \mathrm{C}$.

2. The antibiotic of the bee is distributed in the surface of the cuticle. The head contains more antibiotic than the thorax, and the thorax more than the abdomen. The venom has a slight action and the blood none.

3. In the worker the pharyngeal glands have an antibiotic activity, but the mandibular glands are without action on bacteria.

4. The extract from drones is always inactive. The extract from queens is active, but different from that of the worker. Its activity depends on the queen's age and on the presence of workers. The contact with workers favours the production of antibiotics in the queen. The mandibular glands of the queen are antibiotic, but the pharyngeal glands are not.

5. The antibiotic in the workers is more abundant if they are queenless. The secretion depends both on age and on the physiological condition of the pharyngeal glands, on the seasonal diet (protein), on the race and geographical origin of the bees, and on the pathological condition of the colony.

6. Larvae, pupa, and newly emerged insects do not possess the antibiotic factor, whatever their caste.

7. The antibiotic of the bee is in the fatty acid fraction of the extracts.

8. The antibiotic of the bee is active against 30 strains of bacteria studied. Its activity against Salmonella, Proteus vulgaris and Bacillus alvei is especially interesting.

9. The antibiotic substance extracted from the bee is not fungicidal. On the other hand it strongly inhibits the germination of pollen. This extract also partially inhibits the germination of seeds.

Io. Propolis contains an antibiotic substance, soluble in water and in alcohol, thermostable and stable to ageing. This substance originates in the resin collected from poplar buds, which give an extract with a similar action. This substance has no inhibitory action against Escherichia coli and Pseudomonas pyocyanea; it is however very active against Proteus vulgaris and Bacillus alvei. In addition propolis has powerful antigerminative properties against pollen grains and seeds of higher plants. The propolis extract is the only potent fungicide obtainable from the hive.

II. Wax contains an antibiotic substance soluble in water, very soluble in acetone, and thermostable. It has no effect on Escherichia coli and Pseudomonas pyocyanea but a strong effect on Proteus vulgaris, Salmonella gallinarum and Bacillus alvei. The extract of wax does not contain the fungicidal factor, but it partially inhibits the germination of seeds. 
12. The pollen collected by bees contains a very active antibiotic factor. This factor, which is alcohol- and water-soluble, comes from the plant and from the bee. The antibiotic value of pollen increases as a function of time and of temperature during its storage. Certain pollens are inactive against bacteria, but an average sample of the harvest is always antibiotic. The effect is particularly clear on Salmonella, Proteus vulgaris, E. coli Bordet, E. coli B., Bacillus alvei and Bacillus lavvae. Furthermore it is active against the 30 strains listed in Table I. The extract of pollen has a weak action on the germination of pollen, and partially inhibits the germination of seeds. It has no fungicidal value.

I3. Honey contains an antibiotic substance which is thermostable, photolabile, and which can be extracted by acetone. The greater part of this substance comes from the bee. The activity of this antibiotic is weak in comparison with other hive products, the most sensitive strains being Proteus vulgaris and Bacillus alvei. It has no action on Escherichia coli and Pseudomonas pyocyanea. The extract of honey has no fungicidal activity. On the other hand it is a powerful inhibitor of the germination of pollens and of seeds.

I4. Royal jelly contains an antibiotic factor, soluble in water, alcohol and ether. This substance is very unstabla, but will stand being heated at $120^{\circ} \mathrm{C}$. for 20 minutes. It is the acid fraction of the jelly which is active. The antibiotic activity is weak in comparison with that of the other hive products, nil towards some strains of Escherichia coli and Pseudomonas pyocyanea, weak towards Bacillus larvae, greater towards $E$. coli B., and Proteus rulgaris. Royal jelly is not fungicidal, but it partially inhibits the germination of seeds and of pollens.

I5. None of the hive products contain an antibiotic substance which is identical with that extracted from the body of the bee.

I6. All the antibiotic substances extracted from colonies are active (to a greater or lesser degree) against strains which are pathogenic to brood.

I7. Antibiotic substances occur in other social insects, but it is not a general rule. Similar effects to those with Apis mellifica are found in the bee Meliponula bocandei and its products.

I8. The solitary bee $X$ ylocopa violacea also contains an antibiotic substance.

I9. The investigation of antibiotic substances in various insects has demonstrated their presence or absence in different orders, but it has not been possible to give an explanation of the phenomenon.

\section{ZUSAMMENFASSUNG}

I. Der Körper der erwachsenen Biene enthält eine wasser- und alkohollösliche Substanz, deren Extrakt für Mäuse ungiftig ist. Die Substanz ist thermostabil ; der Extrakt ist nur bei Aufbewahrung bei $0^{\circ} \mathrm{C}$ stabil.

2. Das Antibiotikum der Biene ist auf der Cuticula-Oberfläche verteilt. Der Kopf enthält mehr des Antibiotikums als der Thorax, und diaser mehr als das Abdomen. Das Bienengift hat eine geringe antibiotische Wirkung, im Bienenblut fehlt sie ganz.

3. Bei der Bienenarbeiterin besitzen die Pharynxdriisen eine stark antibiotische Wirkung, die Mandibulardriisen sind wirkungslos gegenïber Bakterien.

4. Der Extrakt aus Drohnen ist stets inaktiv. Der Extrakt aus Königinnen ist aktiv, jedoch verschieden von demjenigen aus Arbeiterinnen. Seine Aktivität ist abhängig vom Alter und der Anwesenheit von Arbeiterinnen. Der Kontakt mit Arbeiterinnen begiinstigt die Produktion von Antibiotika bei 
der Königin. Das Sekret der Mandibulardrïsen der Königin ist antibiotisch wirksam, dasjenige der Pharynxdrüsen ist unwirksam.

5. Das Antibiotikum der Arbeiterinnen ist reichlicher, wenn sie ohne Königin gehalten werden. Die Sekretion hängt ebenfalls ab vom Alter der Bienen, dem physiologischen Zustand der Pharynxdrüsen, der Jahreszeit, der Ernährungsweise (Eiweisstoffe), der Rasse, der geographischen Herkunft und dem pathologischen Zustand des Volkes.

6. Iarven, Nymphen und schliipfende Insekten aller Kasten besitzen keinen antibiotischer Stoff.

7. Das Antibiotikum der Biene ist in der Fraktion der Fettsäuren enthalten.

8. Das Antibiotikum der Biene ist aktiv gegenüber den 30 untersuchten Bakterienstämmen. Besonders stark ist die Wirkung auf Salmonella, Proteus vulgaris und Bacillus alvei.

9. Der aus Bienen extrahierte antibiotische Stoff ist wirksam gegenüber Pilzen, dagegen hemmt er sehr stark die Pollenkeimung und teilweise auch diejenige von Samen.

Io. Das Propolis enthält eine antibiotische Substanz, die wasser- und alkohollöslich, thermo- und altersstabil ist. Diese Substanz stammt von dem auf Pappelknospen gesammelten Harz, das einen Extrakt von ähnlicher Wirkung liefert. Gegenüber Escherichia coli und Psendomonas pyocyanea ist diese Substanz unwirksam; stark wirksam ist sie jedoch gegeniiber Proteus vulgaris und Bacillus alvei. Das Propolis besitzt ausserdem eine stark keimungshemmende Wirkung gegenüber Pollenkörnern und Samen höherer Pflanzen. Der Propolisextrakt ist anderseits der einzige wirksame pilzhemmende Stoff des Bienenvolkes.

Ir. Das Bienenwachs enthält einen antibiotischen Stoff, der wasserlöslich, hauptsächlich aber azetonlöslich und thermostabil ist. Dieser iibt keine Wirkung auf Escherichia coli und Pseudomonas pyocyanea aus, ist dagegen stark wirksam gegenüber Proteus vulgaris, Salmonella gallinarum und Bacillus alvei. Der Wachsextrakt besitzt keinen pilzhemmenden Stoff, verhindert aber teilweise die Samenkeimung.

I2. Der von Bienen gesammelte Pollen enthält einen stark wirksamen antibiotischen Stoff, der alkohol- und wasserlöslich ist und von der Pflanze und der Biene stammt. Die antibiotische Wirkung des Pollens steigert sich im Verlaufe der Lagerung in Abhängigkeit von Zeit und Temperatur. Einige Pollenarten bleiben gegenüber Bakterien unwirksam, eine Durchschnittsprobe der Eirnte ist jedoch stets wirksam. Die Wirkung ist besonders deutlich auf: Salmonella, Proteus vulgaris, E. coli Bordet, E. coli B., Bacillus alvei und Bacillus lavvae. Ausserdem ist Pollen gegenüber den 30 in Tabelle I erwähnten Stämmen wirksam. Pollenextrakt wirkt schwach hemmend auf die Pollenkeimung und hemmt teilweise die Samenkeimung; dagegen besitzt er keine pilzhemmende Wirkung.

I3. Honig enthält eine thermostabile, photolabile, azetonlösliche, antibiotische Substanz, die zur Hauptsache von der Biene stammt. Ihre Wirkung ist im Vergleich zu den andern Produkten des Bienenvolkes schwach; die dafür empfindlichsten Stämme sind Proteus vulgaris und Bacillus alvei. Eine Wirkung auf Escherichia coli und Pseudomonas pyocyanea fehlt. Der Honigextrakt besitzt keine pilzhemmende Wirkung; er hemmt dagegen die Pollenund Samenkeimung sehr stark.

I4. Weiselfuttersaft enthält einen wasser-, alkohol- und ätherlöslichen antibiotischen Stoff. Dieser ist sehr unstabil, erträgt jedoch eine Temperatur von 
I $20^{\circ} \mathrm{C}$ während 20 Minuten. Wirksam ist die saure Fraktion des Weiselfuttersaftes. Seine antibiotische Wirkung ist schwach im Vergleich $\mathrm{zu}$ andern Produkten des Bienenvolkes, fehlt gegenüber einigen Stämmen von Escherichia coli und Pseudomonas pyocyanea, ist schwach gegeniiber Bacillus larvae und stärker gegenüber $E$. coli B. und Proteus vulgaris. Der Weiselfuttersaft wirkt nicht pilzhemmend, hemmt jedoch teilweise die Samen- und Pollenkeimung.

I5. Keines der Bienenprodukte besitzt einen antibiotischen Stoff, der in engem Zusammenhang zu dem aus dem Bienenkörper extrahierten Antibiotikum steht.

r6. Alle aus Bienenvölkern extrahierten antibiotischen Stoffe sind mehr oder weniger wirksam gegeniiber pathogenen Stämmen der Bienenbrut.

I7. Antibiotische Substanzen finden sich auch bei andern sozialen Insekten, doch ist dies keineswegs eine allgemeine Regel. Bei Meliponula bocandei sind ähnliche Wirkungen festzustellen wie bei Apis mellifica (Insekten und ihre Produkte).

18. Fine solitäre Apide, Xylocopa violacea, besitzt ebenfalls eine antibiotische Substanz.

r9. Die Untersuchung verschiedener Insekten auf das Vorhandensein antibiotischer Stoffe zeigte, dass solche Stoffe in verschiedenen Klassen vorkommen oder fehlen, ohne dass es möglich wäre, eine Erklärung dafür zu geben.

\section{REFERENCES BIBLIOGRAPHIQUFS}

Aвbот T (O. D.). - Chemical composition and nutrition value of several Florida honeys. Rep. Fla. Agric. Exp. Sta., 101, I939.

BAER (W. S.). - J. Bone. Joint. Surg., 13, 438, I93I.

Bloom (W. L.), Watson (L. W.), Cromartie (W. J.) et Freed (M.). -- J. Infect. Dis., 80 (I), 4I, I947.

Bösiger. - Communication verbale, I957.

Bourn (A.) et VeLU (H.). - Essais de destruction de Schistocerca Peregrina ol., au Maroc, par 1'emploi des cultures microbiennes (Coccobacillus acridiorum, d'Hereide). Bull. Soc. Path. exotique, Paris VIII, 63864I, I9I5.

Bouin (A.) et VELU (H.). - Essais de destruction du Schistocerca peregrina au Maroc, par les Coccobacillus acridiorum du Dr D'Herelize. Ann. Inst. Pasteur, Paris, XXX, 389-42 I, I9I6.

BRUCE WhITE (P.). - The normal bacterial flora of the bee. $J$. path. and bacteriol. Ig2I.

BUCHHEISTER. - Münch. med. Wschr., 1, I6I2, I935.

BURTENSCHAW (J. L. M.). - The mechanism of self disinfection of the human skin and its appendages. J. Hyg. Camb., 42, I84-2 I0, 1942.

ButenandT (A.) et REmbold (H.). - Ueber den Weiselzellenfuttersaft der Honigbiene. I. Isolierung, Konstitutionsermittlung und Vorkommen der Io-oxy-J2-decensäure. Hoppe-Seyl. Z., 308, $(5 / 6), 284-289,1957$.

Cair.las (A.). - Séance de l'Académie d'Agriculture du 3 mai 1944.

CaIrlas (A.). - Les produits de la ruche. Deuxième édition, 6-287, I945.

Chauvin (R.). - Méthode d'observation continue de la ponte de la reine chez les abeilles. L'Apiculteur, Sect. Sci., 94 (II) : I2, I950.

Chauvin (R.). - Sur le déterminisme de l'effet de groupe chen les abeilles. Physiol. Comp. Oecol., 28, I952.

Chauvin (R.). - Aspects sociaux des grandes fonctions chez l'abeille. I.a théorie du superorganisme. Ins. Soc., 1 (2), I23-I29, I054. 
Chauvin (R.). - I a gelée royale. I. Récolte et conservation. Propriétés physiques. L'A piculteur, Sect. Sci., (2), 7-I2, I055.

Chauvin (R.). - Vie et mours des Insectes. Payot. Paris, I89-I95, I956.

Chauvin (R.). - La gelée royale. Composition biochimique de la gelée royale. L'A piculteur, Sect. Sci., (6), I956.

Chauvix (R.). - Sur les effets physiologiques et thérapeutiques des divers extraits de pollens. Extrait de la revue de Pathologie Générale et de Physiologie Clinique (687), 61 I-623, I957.

Chauviv (R.). - Sur les propriétés physiologiques de certains pollens et spécialement sur un principe hyperglycémiant. Compt. Rend. Acad. Sci., 244, I20-I22, I957.

Chauvin (R.). - Valeur thérapeutique du miel.

Chauvin (R.). - Biologie de l'abeille. Revue générale jusqu'en I956. Ann. de l'abeille, $\mathrm{I}^{\mathrm{re}}$ année, $\mathrm{n}^{\circ} \mathrm{I}, 4 \mathrm{I}-47$, $195^{8}$.

Chauvin (R.). - Some remarks on royal jelly (a reply to the review by T. S. K. Johansson et M. Johansson), Bee World, 40, no 4, 97-98, I 959 .

Chauvin (R.). - La valeur diététique et thérapeutique des produits de la ruche. (Miel. Pollens. Gelée Royale). Extrait de "Produits pharmacentiques "), Vo1. 14, $\mathrm{n}^{\text {as }}$ 5-6-7, p. I-27, I959.

Chauvin (R.), Iefromont (C.), Louveaux (J.) et Vergé (J.). - Sur une substance présente dans le pollen, qui s'oppose au développement de certaines bactéries. C. R. Soc. Biol., CXLVI, 645, I952.

Chauvin (R.) et Grassé (P. P.). - I 'effet de groupe et la survie des neutres dans les sociétés animales. Rev. Scient., 182, 46I-464, I944.

Chauvin (R.) et Lavif, (P.). - Recherche sur la substance antibiotique du pollen. Ann. Inst. Pasteur, 90, 523-527, I956.

Chauvin (R.) et Lavie (P.). - Le vieillissement di pollen emmagasiné par les abeilles et de la gelée royale. Compt. Rend. Acad. Sci., 247, 20402042 , I958.

Chauvin (R.) et Lenormand (E.). - Composition et propriétés du pollen récolté par les abeilles. Bull. Acad. Nation. de médecine (Extrait des $\mathrm{n}^{\circ}$ I et 2), 35, 1957.

Chauvin (R.) et Iouveaux (J.). - Étude macroscopique et microscopique de la gelée royale. L'A piculteur, Sect. Sci., (5) , 33-42, I956.

Chouard (P.). - Dormances et inhibitions des graines et des bourgeons; préparation du forçage ; thermopériodisme. (Cours de physiologie végétale de la Sorbonne, publié par le C. D. U., I956.)

Clausen (M. B.) and Smith (M. N.). - Infection of cockroaches with Herpomyces (laboulbeniales). 2, Hystology and Histopathology. Ann. Entomol. Soc. Amer., 49, 85-93, I956.

Clement (A. L.) et Iches (L.). - La santé par le miel, p. 5-I38, chez les auteurs 34 me Lacépède, Paris, $6^{\mathrm{e}}$ mille, I9I2.

Columelite. - "De arboribus " traduit par Claude Cotereat, Paris, I552, $2^{\mathrm{e}}$ livre, p. $6 \mathrm{I} 2$

DoLI (H.). - Ueber antibakterielle Eigenschaften tierischer und menschlicher Sekrete (Hautsekret, Nasensekret, Bronchialsekret, Speichel, Milch) und pflanzlicher Ausscheidungen (Honig u. a.) Medizinische Klinik, $n^{0}$ I6, I-I2, I938.

Jold (H.) et Chex-Ÿ̈-Hsiaxi. — Zeit. f. Hyg., 89, 266, IgIg.

JOLD (H.), DU (I). H.) et I)ZIAO (S. T.). - - Nachweis antibakterieller hitze-und lichtempfindlicher Hemmungsstoffe (Inhibine) im Naturhonig (Bliitenhonig). Zeit. f. Hyg., 120, I55-167, I938.

IOLD (H.) et KNAPP ('T.). - Ueber inhibierende und modifizierende Wirkungen des Honigs auf Iiptheriebazillen und seine Brauchbarkeit zur Bekämpfung des Diphtheriebazillenträgertums. Zeit. f. Hyg., 130, 323-334, I949. 
Doli (H.) et Witzenhausen (R.). - Ein Verfahren zur Beurteilung der örtlichen inhibitorischen (keimvermehrungshemmenden) Wirkung von Honigsorten verschiedener Herkunft. Zeit. t. Hyg., 141, 333-337, I955.

DUISBERG (H.) et GEIBI,EIN (H.). - Ueber die Kontrolle von Erhitzungsschäden bei Honigen. Zeit. f. Lebensmittel-Untersuchung und-Forschung. 107, (6), 489-50I, I958.

EMERson (A. E.). - The superorganisinic aspect of society. Coll. Intern. C. N.R. S., 34, 333-353, Paris, I952.

FEUPREISI, (R.) et KRAT'S (I'). - - Les particularités tuberculostatiques de la propolis, $X V I I^{\mathrm{C}}$ Congrès International d'A piculture, Bologne-Rome, I5-23 septembre 1958 .

Fi,eming. - Proc. roy. Soc. Biol. Sci., 252, 1922.

Fiorkin (M.), I,OzET (F.) et SARLET (H.). - Sur la digestion de la cire d'abeille par la larve de Galleria mellonella Linn. et sur l'utilisation de la cire par une bactérie isolée à partir du contenu intestinal de cette larve. I.VII (I) octobre. Arch. Inter. de Physiol., 7 I-88, I949.

Firorkin (M.), Loze'T (F.) et SARIET (H.). - Utilisation de la cire d'abeille par une bactérie isolée du contenu intestinal de la teigne de la cire. Journal de Physiologie, 41, I79-I8I, I949.

Foley (E. J.), HERMani (F.) et Ires (S. W.). - 'The effect of $\mathrm{pH}$ on the antifungal activity of fatty acids and other agents. J. Invest. Derm., 8, I-3, I947.

FRAXCO (M.). - Ann. Ig., 51, 394, I94I.

Franco (M.) et Sartori (L.). - Ann. Ig., 50, 216 , in Presse Med., no 68-69, 7 I6, I 940.

Frings (H.), Golberg (E.) et Arentzes (J. C.). - Antibacterial action of the blood of the large milkweed bug. Science, 108, 689-690, I948.

GABBERT. -- Honig als Antiseptikum. Diss. Med. Würzburg, I936.

Gi,ASER (R. W.). - On the Existence of Immunity principles in Insectes. Psyche Boston, Mass., XXV, 39-46, I918.

Grynne Jones (G. D.). - The cuticular waterproofing mechanism of the worker honey bee. J. of Exp. Biol., 32, 95-I09, I955.

Gori.ror (C.). - Recherches de caractéristiques physiques de la gelée royale (G. R.) totale. L'Apiculteur, 100, Sect. Sci., (2-3) I-I6, I956.

Goillot (C.). -- Fitude physique de la conservation de la gelée royale. Compt. Rend. Acad. Sci., Paris, 245, I082-1084, 1957.

Gontarski (H.). - Wandlungsfähiger Instinkt der Honigbiene. Umschau in Wiss. u. Techn., 49, 3I0-312, I949.

Gonzenbach et HofmMann. - Schweiz. med. Wschr., 1, 425, 1936.

Grassí: (P. P.). - La régulation sociale chez les Isoptères et les Hyménoptères. Coll. Intern. C. N. R. S., Paris XXXIV, 323-33I, I950.

Gundes, et Blattrer. - Ueber die Wirkung des Honigs auf Bakterien und Wunden. Arch. t. Hyg., 112 (6), 3I9, I934.

Gwatkin (R.) et Falisis (A. M.). - Canad. J. Research, 16, 343, I938.

Hambi.eton (J. I.). - Propolis culture. - D'après U. S. Patent office. - United States Patent Office, Patented August, 1, I950, Serial no 665, 4I0, I 950 .

HAYDAK (M. H.) et Vivico (F. S.). - The changes in the thiamine, riboflavin, niacin and pantothenic acid content in the food of female honeybees during growth with a note on the vitamin $\mathrm{K}$ activity of royal jelly and bee bread. Ann. Ent. Soc. Amer., 43, 36I-367, I943.

HELIEU (C.). - Contribution à l'étude des propriétés antibactériennes de la gelée royale : Effets bactéricides et antibiotiques de la gelée royale nentralisée. Ann. Inst. Pasteur, 91, 23I-237, I956.

HELLEU (C.). - Nouvelles recherches sur les propriétés antibactériennes de la gelée royale et de quelques substances voisines. Communication $\dot{a}$ la Société de pharmacie de l'Onest, Angirs, I6 nov., p. I-I6, I958. 
D'HERELit (F.). - Sur une épizootie de nature bactérienne sévissant sur les sauterelles au Mexique. Compt. Rend. Acad. Sci., Paris, 152, I4I3I4I5, IQII.

Hess (G.). - Ueber den Einfluss der Weisellosigkeit und des Fruchtbarkeitsvitamins E auf die Ovarien der Bienenarbeiterin. Schreeiz. Bienenzeitung, 2 (I), I942.

Hinglais (H.), Hinglais (M.) et Gau'therie (J.). - Etude du pouvoir bactéricide et du pouvoir antibiotique de la gelée royale. Am. Inst. P’ast., 91, I $27-\mathrm{I} 29$, I 956.

Hitchcoock (J. D.). - A milk digesting enzyme in pollen stored by honeybees. Amer. Bee J., 96 (12), 487-489, 1956.

Holst (E. G.). - An antibiotic from a bee pathogen. Science, 102 (déc. 7), 593-594, I945.

JAUBERT (F.). - Sur 1'origine de la coloration de la cire d'abeilles et la composition de la propolis. Compt. Rend. Acad. Sci., 184, II34-I I36, I927.

JAUBERT (F.). L'origine de la coloration jaune de la cire et du miel (rapport présenté au XII Congrès International d'Apiculture, 6-Io août I939). L'A piculteur, $84^{\mathrm{e}}$ année, no $\mathrm{I}, 6-\mathrm{IO}$, I940.

Johansson (R. S. K.) et Johansson (P.). - Royal jelly. Bee World, 39 (Io), 254-264 et Bee World, 39 (II), 277-286, I958.

Kilvai,kina (B.P.). - (Article repris du Pchelovostvo no 10, 1948, citće par le Journal Yougoslave l'Apiculteur no $\mathrm{I} / 3,1950)$, d'après un article de A. Chvidchenko (l'A piculteur de déc. I 950 ).

Kitzes (G.), Schuette (H.) et Elvehjem (C. A.). - The vitamins in honey. J. nutrition, 26, 24I-250, I943.

KoIDsum $(\mathrm{K}$.$) . - Antifungal action of cuticular lipids in insects. J. of inset$ physiology, (I), 40-5I, I957.

Krasikova (V. I.). - Bactericidal properties of brood food. Pchelovodstro $32(8), 50-53,1955$.

Kustenmacher. - Cité pat A. Caillas. Les produits de la ruche, $2^{\mathrm{e}}$ édition, 241, 1947

Kuwabara (M.), - Ueber die Regulation im weisellosen Volke der Honigbiene, besonders die Bestimmung des neuen Weisels. J. Fac. Sci. Univ. Hokkaïdo, 9, 359-38I, 1947.

Landau et Schiemann. - Zeit. $t$. Hyg., 88 I29, I9I9

I ANGLADE (H.), Hingiais (H.) et Hinglais (M.) - Étude du pouvoir microbicide de la gelée royale sur le bacille de Koch. Ann. Inst. Pasteur, 93, 272-276, 1957 .

LARREY (D. I.). - Relation historique et chirurgicale de l'expédition de l'armée d'Orient en Igypte et en Syrie. Clinique chirurgicale, Paris, I829.

LAVIE (P.). - Étude des substances antibiotiques présentes chez Apis mellifica et chez quelques insectes sociaux. Compt. Rend. Acad. Sci., 244, $2653-2655$, I957.

LAVIE (P.). - Action antibiotique de quelques substances provenant de 1'abeille ou de la ruche sur Bacillus larvae et Bacillus alvei. Compt. Rend. Acad. Sci., 248, 455-457, 1959.

LAvie (P.) et PaIN (J.). - Les rapports entre la substance antibiotique des reines et des ouvrières d'abeilles, le développement ovarien et l'ectohormone. Compt. rend. Acad. Sci., 248, I5\$7-I589, I959.

LAVIE (P.) et PaIn (J.). - Relation entre la substance attractive, le facteur antibiotique et le développement ovarien chez la reine d'abeille, $A$ pis mellifica. Compt. Rend. Acad. Sci., 248, 3753-3755, I959.

LAVIE (P.) et Roth (M.). - Sur le thermopréférendum et la production de chaleur chez les abeilles. Phys. Comp. Oecol., III, 57-62, I953.

LECOMTE (J.). - Recherches sur la formation de la grappe chez les abeilles. Z. verlg. Physiol., 32, 499-506, I950. 
LEEFEBURES (C. L.). - Penetration and development of the fungus Beauvaria bassiana in the tissues of the corn borer. Ann. Bot., 48, 44I-452, I934.

I EMAY (P.). - Sur la sécrétion possible d'antibiotique par certains insectes. Revue de pathologie comparée et d'hygiène générale, 373, 589-590, I947.

Leunnberger (F.). - Les abeilles, Payot édit., Paris I929.

I.INDAUER (M.). - Ein Beitrag zur Frage der Arbeitsteilung im Bienenstaat. Z. vergl. Physiol., 34, 299-345, I952.

Lochhead (A. G.). - Mikrobiologie des Honigs. American honey producer 5 , I93I.

Lochhead (A. G.) et FAREli. - Ueber Bakterien in Honig. Canad. J. Res., I930 et I93I.

Lotmar (R.). -- I946, cité par Toumanoff, Les maladies des abeilles, p. I5I, I95I.

I.OUVEaUX (J.). - Recherches sur la récolte du pollen par les abeilles (Apis mellifica L.). Thèse, p. I-206, Ann. de l'A beille, 1958.

MAC Cleskey (S. C.) et Melampy (R. M.). - Bactericidal properties of royal jelly of the honeybee. J. Econ. Ent., 32, 581-587, 1939.

Mac Cleskey (S. C.) et Melampy (R. M.). - J. Bact., 36, 324, I938.

MAC GREGOR (S. E.). - Collection and utilization of propolis and pollen by caged honey bee colonies. American bee journal, 92, 20-2I, I952.

Marchionini. - Klin. Wschr., 924, I 929.

Marchionini - Arch. Derm., 158, 290, I 929.

Matuka (S.) et Topolnik (E.). - The significance of larval food in the ethiology of american and european foulbrood. Vet. Archiv., 22 (3/4), I4I-I56, I952.

MaUrice (A.). - Lucilia sericata en thérapeutique. Larves vivantes, sucs extraits de larves. Résultats cliniques. Thèse fac. Med. Paris, Edit. Lagrand, Paris, I-I07, I934.

Maurizio (A.). - Pollenernährung und Lebensvorgänge bei der Honigbiene (Apis mellifica). Landw. Jahrb. Schweiz., 68, I I5-I82, I954.

Maurizio (A.). - Substances dans le corps de l'abeille mellifique qui empêchent la germination du pollen. Communication au $X V I I^{\mathrm{e}}$ Congrès International d'Apiculture. Bologne-Rome, I5-23 septembre I958.

Maurizio (A.) et Gubler (H. U.). - Ueber die Wirkung von Honig, Futtersaft und Kittharz auf Bakterien. Schweiz. Landw. M. H., 29 (9), 3093I4, I05I.

Métalnikov (S. J.). - - L'infection microbienne et l'immunité chez la mite des abeilles, Galleria mellonella. Monog. Inst. Pasteur. Masson et Cie, Paris 1927.

MétchnikorF (E.). - L'immunité dans les maladies infectieuses. Paris. Igor.

MEYER (W.). - Die "Kittharzbienen " und ihre Tätigkeit. Zeit. f. Bienenforsch., 2 (7), I85-200, I954.

MORELLINI (M.). - Activité bactéricide de la gelée royale, Communication au $X \mathrm{I}^{\prime} I I^{\mathrm{e}}$ Congrès International d'A piculture, Bologne-Rome, 15-23 septembre, I958.

Mukerji (D.) et Chatterjee (S. W.). - Permeability of insect cuticules to aqueous stains and rulnerability of insecticides. Nature (London), I7I, II9-I20, I953.

Nutini (L. G.) et Kreke (C. W.). J. Bact., 44, 66r, I942.

Olivier (H. R.). - Antibiotic action of an extract of Galleria mellonella, Nature, 159, 685, I947.

ORTEL (S.) et MARKWARD'T (F.). - Untersuchungen über die antibakteriellen Eigenschaften des Bienengiftes. Pharmazie, 10 (I2), 743-746, I955.

PAdDock (F. B.). - Disease resistant stock. Iowa State Port. Soc. Rept. aug., 9, p. 24I-243, I950.

PAIliot (A.). - L'immunité naturelle chez les insectes. Étude d'un cas d'immunité humorale. Compt. Rend. Acad. Sci., Paris, 169, I I22, I9I9. 
Pailiot (A.). - Mécanisme de l'immunité humorale chez les Insectes. Compt. Rend. Acad. Sci., Paris, 172, 546, Ig2 I.

Pailior (A.). - Une science nouvelle: 1'immunologie comparée. Rev. Gén. Sc. $35^{\mathrm{e}}$ année, p. $422-429$, I924.

PAILIOT (A.). - L'infection chez les Insectes. Imprimerie de Trévoux, p. 6-535, I933.

PAIN (J.). - L'alimentation et le développement des ovaires chez l'ouvrière de 1'abeille (Apis mellifica L.). Arch. Inst. Physiol. LIX (2) 203-2Io, I95I.

PaIn (J.). - Sur l'ectohormone des reines d'abeilles. Insec. Soc., 3 (I), Ig9202, I95I.

PAIN (J.). - Communication au Congrès de l'U. I. E. I. S., Paris, du 9 au I3 juillet I957.

PaIN (J.). - Communication orale, I959.

Parḱ (A.). - Les œuvres d'Ambroise Paré. Ed. G. Buon, 2e édit., Paris, I579.

PARK (O. W.). - Disease Resistance and American Foulbrood. Amer. Bee Journal. LXXVI, I2-I5, I936.

PARKER. - The collection and utilization of pollen by the honeybee. Cornell Univ., 98 p., I926.

Parvel. - Cité par A. Cailias (Les produits de la ruche, $2^{\mathrm{e}}$ édition), p. 244.

PAsteur (L.). - Cité par A. Pailloto - L'infection chez les insectes, I933. (Introduction), I874.

Pavan (M.). - Ricerche sugli antibiotici di origine animale, Anno $19^{\circ}, \mathrm{n}^{\circ} 9$, Extrait de : La ricerca scientifica, I949.

Pavan (M.). - Sugli antibiotici di origine animale. Bolletino dell'Istituto Sieroterapico Milanese, XXXI( III-IV), I95-208 (V-VI), 232-345, I952.

Pavan (M.). - Studi sugli antibiotici e insecticidi di origine animale. I. Sul principo attivo della larva di Melasoma populi L. (Col. chrysomelidae). Archivio zoologico italiano, vol. XXXVIII, I953.

PELLET (F. C.). - Solving the disease problem. Amer. Bee Journal, 75 (2), Feb. I935.

Perret Maisonneuve (A.). - Compt. Rend. Acad. Sci., 185, I3I7, I927.

Pertzoff (V.). - Sur la lipase des Chenilles de Galleria mellonella, Compt. Rend. Acad. Sci., 18\%, 253, I928.

PhILIPP (P. W.). - Zur Kittharzfrage : Die Desinfektionskraft des echten Kittharzes. Dentsche Illustrierte Bienenzeitung : LXVI, (6), I07-Io9, I929.

PhILIPP (P. W.). Die wirtschaftliche Bedeutung des Kittharzes. Die deutsche Bienenzucht in Theorie und Praxis. XIII (5), 89-90, I935.

Phir.ipp (P. W.) et Weck. - Cités par G. J. Peynet (Remarque sur la propolis, "l'Apiculture française", mars I928).

RAIGNIER (A.). - L'Économie thermique d'une colonie polycalique de la fourmi des bois (Formica rufa polyctena Forst). Ext. de "la cellule" LI (3), I948.

REHM (E.). - Klin. Wschr., 26 (7/8), I20, I948.

v. RHein (N. W.). - Ueber die Entstehung des weiblichen Dimorphismus im Bienenstaat. W. Roux. Archiv. t. Entwicklungsmech., 129, 601-665, I933.

Rinbands (C. R.). - Division of labour in the honeybee community. Proc. roy. Soc., 140, 32-43, I952.

Richards (G. A.). - Sonderdruck aus Ergebnisse der Biologie. Bd XX Heidelberg (publication $n^{\circ}$ 943, Micellaneous J. Series, Minnesota A gricultural Exp. Station. St Paul, I, Minnesota U. S. A.), I-26, I958.

Root (A. I.). - ABC and XYZ of bee culture. Root Company, Medina, U. S. A. (Editeur), I-703, I954.

Rösch (G. A.). - Arbeitsteilung im Bienenstaat. Z. vergl. Physiol., 2 (6), 57 I -63 I et 12 (I), I-7I, I 925 .

Annales des Abeilles. - ig6o. 
Rösch (G. A.). - Beobachtungen an Kittharz sammelnden Bienen (Apis mellifica L.). Biol. Zentralblatt, 47, II3-I2I, 1927.

Rothmann (S.), Sminjanic (A. M.) et Shapino (A. L.). - Fungistatic action of hair fat on Microsporon audouini. Proc. Soc. Exp. Biol., N. Y., 60, 394-395, I945.

Ro'thmann (S.), Smiljanic (A. M.) et Weitkamp (A. W.). - Mechanism of spontaneous care in puberty of ringworm of the scalp. Science, 104, 2OI-203, I 946.

Roubaud (E.). - La désocialisation chez l'abeille domestique (Apis mellifica L.). Coll. Internat. C. N. R. S., I950 (Paris, I952, 34, 293-296).

Ror, - I937, Cité par M. PAVAN (Sugli antibiotici di origine animale. Bollettino dell'Istituto Sieroterapico Milanese XXXI (III-IV), I95-208, (V-VI), 232-245, I952.)

SACKeTt. - Bulletin no 252 de la Station de Bactériologie de l'A gricultural College du Colorado, cité par Root (ABC and XYZ of bee culture), I954.

Schade (J.), Marsh (G.) et EckerT (J.). - Improved methods of determining diastase and hydroxy-methyl-furfural in honey and their relationship to the bacteriostatic quality of honey. Communication 10th International Congress of Entomology. Montreal, August I7-25, 1956.

Schuler (R.) et VOGEL (R.). - Wirkstoffe des Bienenhonigs. Arzneimitt. Forsch., 6, 66I-668, I956.

Shikata (M.). - Studies on the structure of the integument of the silkworm with special reference to its cuticular lipids. II. In the physico chemical natures of the ether extractives and their components obtained from the exuviae. J. Seric. Sc. Tokyo, 23, 35-43, I954.

Shuer (S. E.) et Dixon (R. W.). - Can.J. Zool., 36, I958.

SLIFER (E. H.). - Vulnerable aeras on the surface of the tarsus and pretarsus of the grasshopper. Ann. Entom. Soc. Amer., 43, I73-I88, I950.

Smith (E. L.), BEck (J. V.) et ANDERSon (E. J.). - The effect of pollen on sporulation of Bacillus larvae (WHITE). Journ. of Bact. Ent., 57, 2I3$2 \mathbf{1} 8, \quad$ I949.

SNodgrass (R. E.). - Anatomy and Physiology of the Honey Bee. Mc. Graw-Hill, Book Cie Nere-York, I925.

Spöttel (W.). - Honig und Trockenmilch. J. Ambrosius Barth Verlag, Leipzig, I950.

Steinhaus (E. A.). - Principles of insect pathology, Mc. Graw-Hill, Book Cie, New York, Ire édition I949.

Steinke (L.). - Neue Vorschriften zur Prüfung der Honigdiastase nach Koch. Zeit. 1. Bienenk., IV (2), 38-40, I957.

STokor. (W. W.). - The rancidity of coconut ail produced by mould action. Biochem. J., 22, 80-93, I928.

STUmper (R.). - Études myrmécologiques. VIII. Examen chimique et microbiologique de quelques nids de Lasius fuliginosus Latr. Arch. Inst. Grand Ducal de Luxembourg, 19, 243-250, I950.

STURTEVAN'T (A. P.). - A study of the behaviour of bees in colonies affected by european foulbrood. U. S. Dept. Agric. Bull., ñ 804 , I920.

Sussman (A. S.). - Studies of an insect mycosis. 3. Histopathology of an aspergillosis. Ann. Entomol. Soc. Amer., 45, 233-245, I952.

Svoboda (J.) - Ueber den Wert des Pollens als Nahrungsmittel für Bienen. Schweiz. Bienen Z., 4, x940.

Synge (A. D.) - Pollen collection by honeybees. J. of Animal Ecology, 16 (2), $\mathrm{x} 22-138, \mathrm{I} 947$.

TARR (FI. L. A.) - Summary of foulbrood disease Investigation. Report May Ist to September 3oth. Bee World, 12 I934.

Tausson (W. O.) - Ueber die Oxydation der Wachse durch Mikroorganisme Bioch. Zeitschr., IXXXV, I93, I928. 
Trmon-David (J.). - Ann. Fac. Sc. Marseille, 13 (4), I, I940; 15 (2), 49, I94I ; 16 (4), I79, I945.

Timon-David (J.). - Ann. Biol., 21 (I0-20), I33, 1945.

Timon-David (J.). - Ann. Biol., 23 (9-Io), 237, I947.

Toon et Bretherick. - The composition of pollen. J. Econ. Entom., 35 (3), 2I2-3I7, I942.

ToumanofF (C.). - Essais sur l'immunisation des abeilles. Compt. rend. Acad. Sci., 185, I078, I927.

ToumanofF (C.). - Note sur l'infection du couvain des abeilles par Bacillus larvae. Bull. Acad. Vétér. France, II (I), 45, I929.

TOUMANOFF (C.). - Les maladies des abeilles. Edit. par la Revue Française d'A piculture, 68, I, 325, I95I.

VAgo (C.). - L'enchaînement des maladies chez les insectes. Thèse, p. II8r, Ann. des Epiphyties, I959.

VERGÉ (J.). - L'activité antibactérienne de la propolis du miel et de la gelée royale. L'Apiculteur, 95 (6), I3-20 (ro), I95I.

Vuillaume (M.). - Les substances inhibitrices de la construction des cellules royales chez les abeilles. Compt. rend. Acad. Sci., 246, I298-I299, 1958.

WARNECKE (B.) et DUISBERG (H.). - Die bakteriostatische (inhibitorische) Wirkung des Honigs. Zeit. f. Lebensmittel-Untersuchung und-Forschung, 107, 340-344, I958.

WEAVER (N.). - Rearing of honeybee larvae on royal jelly in the laboratory. Science, 8, 121, 509-5I0, I947.

Weitkamp (A. W.), Suiljanic (A. M.) et Rothman (S.). - Free fatty acids of human hair. J. Amer. Chem. Soc., 69, I936-1939, 1947.

White (G. F.). - The bacteria of the apiary. U. S. Depart. of Agriculture, Technical series $\mathbf{n}^{\circ} \mathrm{I} 4$, 1906 .

WigGlesWORTH (V. B.). - The principles of insect physiology. Methyen a. Co. Ltd., London, I-544, I950.

Woodrow (A. W.). - Susceptibility of honeybee larvae to american foulbrood. Gleanings in Bee Cult., 69 (3), I48-I5I, I94I.

WYss (O.), Ludwig (B. J.) et JoINER (R. R.). - The fungistatic and fungicidal action of fatty acids and related compounds. Arch. Biochem., 7, 4I5-425, I 945 .

ZAIss. - Miunch. Med. Wschr., II, I89I, I934. 